University of Louisville ThinkIR: The University of Louisville's Institutional Repository

\title{
Identification and biochemical characterization of the CHLAMYDIA TRACHOMATIS type III secretion chaperone, SLC1, and its role in the translocation of the invasion-associated effector TARP.
}

Amanda J. Brinkworth

University of Louisville

Follow this and additional works at: https://ir.library.louisville.edu/etd

\section{Recommended Citation}

Brinkworth, Amanda J., "Identification and biochemical characterization of the CHLAMYDIA TRACHOMATIS type III secretion chaperone, SLC1, and its role in the translocation of the invasion-associated effector TARP." (2011). Electronic Theses and Dissertations. Paper 153.

https://doi.org/10.18297/etd/153

This Doctoral Dissertation is brought to you for free and open access by ThinkIR: The University of Louisville's Institutional Repository. It has been accepted for inclusion in Electronic Theses and Dissertations by an authorized administrator of ThinkIR: The University of Louisville's Institutional Repository. This title appears here courtesy of the author, who has retained all other copyrights. For more information, please contact thinkir@louisville.edu. 


\title{
IDENTIFICATION AND BIOCHEMICAL CHARACTERIZATION OF THE CHLAMYDIA TRACHOMATIS TYPE III SECRETION CHAPERONE, SLC1, AND ITS ROLE IN THE TRANSLOCATION OF THE INVASION- ASSOCIATED EFFECTOR TARP
}

\author{
By \\ Amanda J. Brinkworth \\ A Dissertation \\ submitted to the Faculty of the \\ Graduate School of the University of Louisville \\ in Partial Fulfillment of the Requirements \\ for the Degree of \\ Doctor of Philosophy \\ in Microbiology \\ Department of Microbiology and Immunology \\ University of Louisville \\ Louisville, Kentucky, USA
}

May 2011 


\section{IDENTIFICATION AND BIOCHEMICAL CHARACTERIZATION OF THE CHLAMYDIA TRACHOMATIS TYPE III SECRETION CHAPERONE, SLC1, AND ITS ROLE IN THE TRANSLOCATION OF THE INVASION-ASSOCIATED EFFECTOR TARP}

By

Amanda J. Brinkworth

A Dissertation Approved on

January 27, 2010

by the following Dissertation Committee:

Michah Worley

Yousef Abu-Kwaik

Richard D. Miller 


\title{
DEDICATION
}

\author{
I would like to dedicate this dissertation to \\ my loving parents \\ Pam and Greg \\ and \\ my amazing husband \\ Jeff,
}

Without your love and support I never would have been able to accomplish my goals. 


\section{ACKNOWLEDGEMENTS}

I would like to express my most sincere gratitude to my mentor Dr. Rey Carabeo, who has consistently gone above and beyond my expectations to help me in both science and life. I would also like to acknowledge my co-mentor Dr. James Graham, whose guidance and generosity have been pivotal to my work. My committee members Dr. Richard Miller, Dr. Yousef Abu-Kwaik, and Dr. Michah Worley all provided essential discussions and suggestions throughout my thesis, and I want to specifically thank you for all of your time and efforts. I want to gratefully acknowledge our collaborator, Dr. Richard Hayward at University College London for his invaluable advice and efforts toward all of the gel filtration chromatography work in this dissertation.

Furthermore, I want to specifically thank the Imperial College London masters and undergraduate students Antonio Tedim Pedrosa, Sevanna Shahbazian, and Katarzyna Roguska, who worked with me to complete the bacterial 2-hybrid and translocation studies. Their hard-work and enthusiasm was greatly appreciated. I would like to thank all of the former and current lab members: Jennifer, Illya, Chris, Scot, Tristan, Sophie, Denise, and David for their kindness, patience, and supportiveness throughout my thesis.

I specifically want to thank Sophie Nicod for being such a lovely person who always was there for me whenever lab or life became chaotic. Gunnar Schroeder, Alex Wong, Diana Munera, and Robert Fagan from the Centre for Molecular Microbiology and Infection at Imperial College London and Chris Price at University of Louisville were all excellent resources and were very helpful during my thesis. I especially want to acknowledge my best friend, Meena Vanchinathan, whose sense of humor and companionship has been essential to my graduate career. Finally I would like to acknowledge my wonderful and loving husband Jeff, who can never be thanked enough for his endless patience and support. 
ABSTRACT

\section{IDENTIFICATION AND BIOCHEMICAL CHARACTERIZATION OF THE CHLAMYDIA TRACHOMATIS TYPE III SECRETION CHAPERONE, SLC1, AND ITS ROLE IN THE TRANSLOCATION OF THE INVASION- ASSOCIATED EFFECTOR TARP}

Amanda Brinkworth

May 2011

Chlamydia trachomatis is an obligate intracellular pathogen that utilizes a type III secretion system to enter mammalian cells and establish an intracellular niche. TARP, the translocated actin recruitment protein, is a chlamydial invasion protein known to be type III secreted by the metabolically inert elementary body upon docking to the mammalian cell surface. Because immediate secretion of TARP into host cells is necessary for entry, I hypothesized that a chlamydial chaperone binds to TARP and facilitates its translocation through the type III secretion apparatus. Most effectorbinding type III secretion chaperones are small (14-18 kDa), have an acidic pI, and share a specific secondary structure of alternating alpha-helices $(\alpha)$ and beta-sheets $(\beta)$.

Typically, type III secretion chaperones dimerize and interact with their effectors as a complex of two molecules of chaperone to one effector molecule. Only 3 Chlamydia trachomatis proteins have been identified in EB's that are predicted to be putative chlamydial type III effector chaperones. These are CT043, CT663, and CT088, which I have designated as Slc1, Slc2, and Scc1, respectively. These chaperones were tested for their interaction with the N-terminal 200 amino acids of TARP $\left(\mathrm{HIS}_{6}\right.$-TARP ${ }^{1-200}$ ) by co- 
immunoprecipitation. $\mathrm{HIS}_{6}-\mathrm{TARP}^{1-200}$ interacted specifically with Slc1, but not Scc1 or Slc2. This interaction was enhanced by coexpression of the recombinant proteins. To confirm this interaction and rule out the possibility of Slc1 heterodimerization enhancing the interaction with TARP, I employed a 2-hybrid system to test for TARP: chaperone and chaperone:chaperone interactions. I confirmed the specific interaction between $\mathrm{Cya}_{18}$-TARP ${ }^{1-200}$ and $\mathrm{Cya}_{25}$-Slc1. I was also able to detect Slc1 interaction with itself as well as confirm a few other previously described chaperone-chaperone interactions. Analysis by crosslinking and gel filtration chromatography indicated that Slc1 forms a stable dimer in solution. Complexes of the Slc1 chaperone dimer with TARP in a 2:1 stoichiometry were detected following purification from co-expressing bacteria, but not following addition of singly purified species. Expression of beta-lactamase fused to $\mathrm{TARP}^{\mathrm{I}-200}$ by the heterologous system Yersinia enterocolitica allowed for secretion of TARP into type-III inducing media (low calcium). Furthermore I was able to detect Slc1-dependent translocation of TARP into HeLa cells via the heterologous type III secretion system of $Y$. enterocolitica, and also by the SPI-2 system of Salmonella enterica serovar typhimurium. 


\section{TABLE OF CONTENTS}

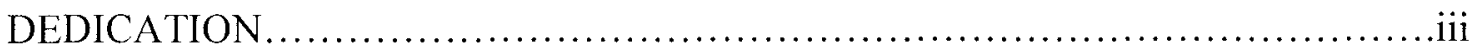

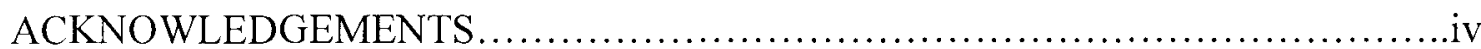

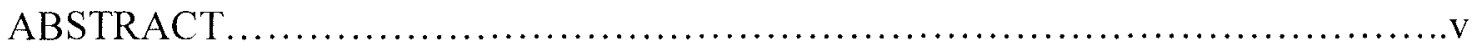

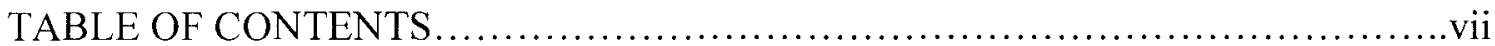

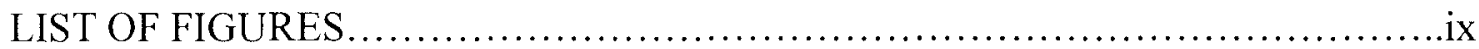

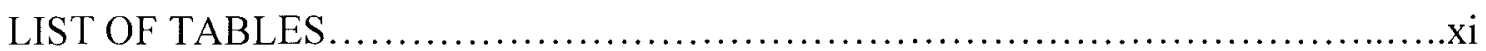

\section{CHAPTER 1: INTRODUCTION}

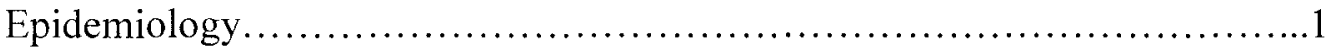

Chlamydia trachomatis pathogenesis.................................. 3

Adaptation of pathogenic Chlamydia to their mammalian hosts................5

Type III Secretion...................................................6

Type III secretion and Chlamydia's developmental cycle....................14

Attachment ............................................................

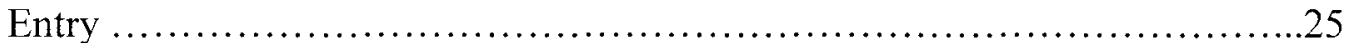

Hypothesis................................................... 28

\section{CHAPTER 2: MATERIALS AND METHODS}

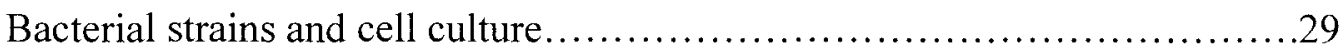

Plasmid construction................................................... 31

Protein expression, lysate preparation, and protein purification...............32

Co-immunoprecipitation assays and immunodetection......................33

$\beta$-galactosidase assay............................................. 33

Colocalization assay ..................................................... 34

Yersinia Translocation Assay........................................ 35

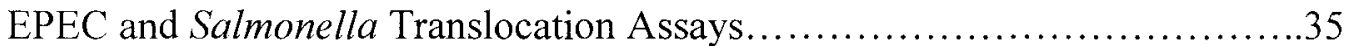

CHAPTER 3: IDENTIFYING INTERACTIONS BETWEEN TARP AND TYPE III CHAPERONES FROM CHLAMYDIA TRACHOMATIS 
Interaction of the $\mathrm{N}$-terminal 200 amino acids of TARP with the putative

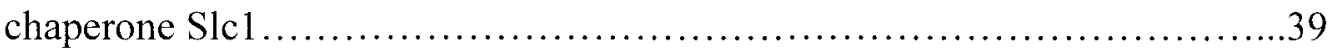

Slc1 can interact with TARP from other Chlamydia.......................58

CHAPTER 4: IDENTIFICATION AND CHARACTERIZATION OF CHLAMYDIAL CHAPERONE:CHAPERONE INTERACTIONS

Discovery of chlamydial chaperone:chaperone pairs........................64

Multimerization of Sc1 homo-complexes..............................72

CHAPTER 5: FUNCTIONAL DETERMINATION OF SLC1 AS A TYPE III SECRETION CHAPERONE FOR TARP

TARP-Slcl complexes indicate the expected 2:1 stoichiometry of type III chaperone: effector: interactions........................................76 Translocation of TARP by heterologous type III secretion systems..........81

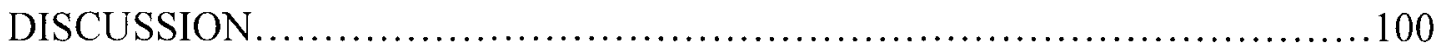

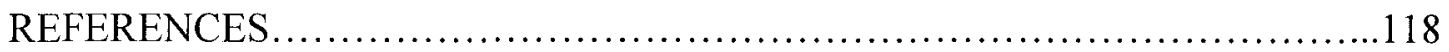

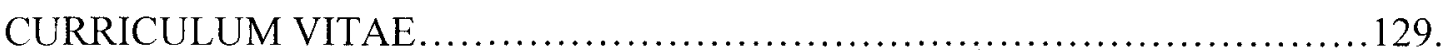




\section{LIST OF FIGURES}

Secondary structure alignment of type III chaperones...............................38

Amplification of TARP inserts for cloning into pET200..........................41

Colony PCR of transformants of pET200-TARP200aa...........................42

Amplification of chaperone inserts for cloning into pET200.....................43

Colony PCR of transformants of pET101-chaperones.............................44

Pull-down of TARP by chlamydial chaperones from singly expressed lysates..........46

Pulldown of chaperones by TARP following coexpression.........................48

Diagram of the pUT18C and pKT25 $\beta$-lactamase fusion vectors...................50

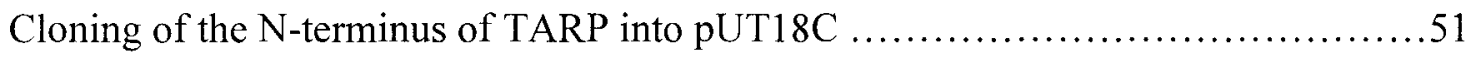

Confirmation of pKT25-chaperone transformants..............................52

Diagram of the E.coli 2-hybrid system.............................................53

Bacterial 2-hybrid interactions between TARP and chaperones on indicator plates.....55

$\beta$-galactosidase activity of TARP:chaperone bacterial 2-hybrid interactions...........57

Amino acid alignment of the $\mathrm{N}$-terminus of TARP from different Chlamydiae ........60

Diagram of recombination vectors used for mammalian expression.................61

Localization of Slc1 to full-length $C$. caviae TARP aggregates.....................62

Pulldown of the N-terminus of C. muridarum and C. pneumoniae TARPS with Slc1..63

Pulldown of Slc1 and Slc2 in the presence of Slc1 ............................66

Pulldown of Slc1 in the presence of Scc1....................................67 
Bacterial 2-hybrid interactions between chlamydial chaperones.

Quantitative analysis of bacterial 2-hybrid chaperone:chaperone interactions..........71

Inducing multimerization of Slc1 by crosslinking.............................. 73

Detection of native Slc1 species by gel filtration chromatography.................75

Native gel shift of TARP:Slc1 complex ...................................

Detection of TARP:Slc1 native complex (Coexpressed).......................... 79

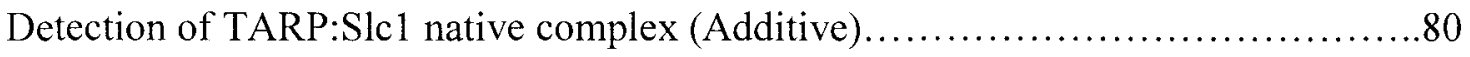

Diagrams of the bacterial expression vectors $\mathrm{pBAD} 18-\mathrm{Cm}$ and $\mathrm{pCX} 340 \ldots \ldots \ldots \ldots . . . .82$

Cloning chlamydial chaperones into the pBAD18-Cm vector........................83

Adding a KpnI site to the pET200-TARP200 construct by SLIM PCR..............84

Cloning TARP into the $\mathrm{pCX} 340$ vector........................................ 85

Diagram of the CCF2/AM fluorescence-based translocation assay $\ldots \ldots \ldots \ldots \ldots \ldots . . . . . . .87$.

Secretion of TARP by Yersinia enterocolitica ................................... 89

Translocation of TARP by Yersinia enterocolitica............................ 90

Translocation of TARP by Enteropathogenic $E$. coli.............................92

Translocation of TARP by Salmonella typhimurium SPI-1 and SPI-2 ...............94

Phylogenetic analysis of multiple type III components from bacterial pathogens.......96 


\section{LIST OF TABLES}

Type III chaperones and their substrates........................................13

Expression of type III genes during the chlamydial developmental cycle...............18

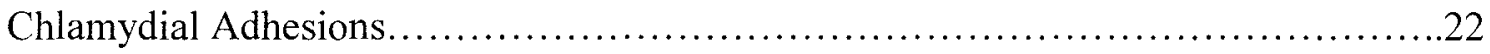

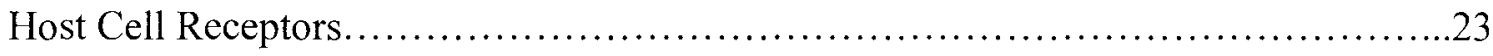

Strains and Plasmids used in this study ........................................97

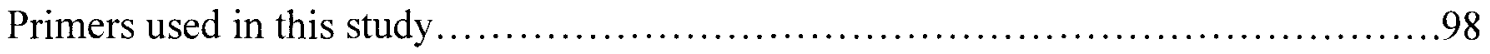




\section{CHAPTER I}

\section{INTRODUCTION}

\section{Epidemiology}

A myriad of diseases are caused by pathogenic Chlamydia species that can efficiently invade human mucosal epithelial cells. Genital infections of the superficial mucosal epithelium in the endocervix or urethra by serovars D-K can result in cervicitis, urethritis, endometritis, salpingitis, and proctitis. LGV1-3 of C. trachomatis are responsible for invasive genital infections that result in lymphogranuloma venereum, and serovars A-C are responsible for ocular infections that can result in blinding trachoma. Lung infections with C. pneumoniae or C.psittaci can result in mild upper respiratory or life-threatening pneumoniae, respectively. Chlamydial infections are also endemic to koalas, guinea pigs, mice, sheep, and cattle.

Chlamydia is the most common bacterial sexually transmitted disease in the United States. Most Chlamydia infections are asymptomatic, allowing the bacteria to persist in the genital tract until it is cleared naturally by the host immune system. In a small fraction of infected females, long-term complications such as pelvic inflammatory disease (PID), ectopic pregnancy, and tubal infertility occur[1]. An extensive survey of Chlamydia genital infections in 2007 was reported by the Center for Disease Control (CDC) and they estimated over 1.1 million infections in the U.S. Their most convincing findings were from the screening of the National Job Training applicants that reported 
infection rates of $13.2 \%$ of females and $7.2 \%$ of males aged $16-24$ year olds Were positive for C. trachomatis infections[2]. Furthermore the World Health Organization has estimated over 90 million cases of C. trachomatis infection worldwide[3], with serovars $\mathrm{D}, \mathrm{E}$, and $\mathrm{F}$ being the most prevalent $[4,5]$.

C.trachomatis is also the causative agent of blinding trachoma that accounts for $16 \%$ of blindness worldwide. Ocular infection mainly affects young children $(0-4$ yrs old) and is cleared without complications within 2-4 weeks. However familial transition to adults can result in serious scarring of the tarsal conjunctiva, inversion of the eyelid, and corneal scarring due to scratching by the in-turned eyelashes. Without treatment, continued irritation by chlamydial antigens will lead to corneal opacity and eventually blindness[6]. Trachoma is mainly a disease endemic to poverty-stricken villages with little access to clean water and medicine.

The World Health Organization currently funds a campaign to eradicate blinding

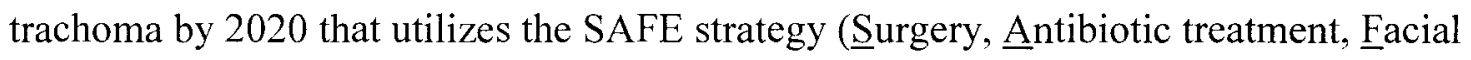
cleanliness, and Environmental improvement)[7]. Some advanced ocular infections require eyelid surgery in addition to antibiotic treatment to prevent blindness. Treatment with tetracycline derivatives (doxycycline) or macrolides (erythromycin, azithromycin) is sufficient to clear up most C.trachomatis infections. Mass treatment with antibiotics has been proposed to eradicate trachoma within specific communities, but evidence of pneumococcal resistance to these antibiotics has emerged as a major barrier to mass treatment strategies[8].

C. pneumoniae is responsible for $10 \%$ of mild upper respiratory infections. It has been estimated that $80 \%$ of people will have had C. pneumoniae infection in their 
lifetime[9]. C. pneumoniae has also been found in the joints and in atherosclerotic plaques, associating the bacterium with chronic inflammatory diseases as well[10].

\section{Chlamydia pathogenesis}

The ability of Chlamydia trachomatis to cause damage to human tissues is a consequence of the host immune response to infection. Clearance of C.trachomatis genital infections involves a combination of innate and cellular immune responses[11, 12] The housekeeping gene, heat-shock protein 70 (Hsp70), from Chlamydia species is recognized by host cell toll-like receptors TLR2 in cooperation with CD14 to induce secretion of the pro-inflammatory cytokine IL-8 (interleukin-8)[13]. Initial infection of human epithelial cells results in the release of pro-inflammatory cytokines such as IL-1 $\beta$, IL-6, GM-CSF (granulocyte-macrophage colony stimulating factor) and TNF (tumor necrosis factor) that attract monocytes, natural killer cells and neutrophils, as well as in releasing tissue-damaging matrix-metalloproteases (MMPs) $[1,13]$. These recruited cells further release damaging MMP's, elastase, and secrete IFN- $\gamma$ (interferon-gamma) to drive a Th1-like $\mathrm{CD} 4^{+} \mathrm{T}$-cell response. Further mediators of cellular immunity $\left(\mathrm{CD} 8^{+} \mathrm{T}\right.$-cells, B cells, and plasma cells) are recruited to the site of infection and secrete IFN- $\gamma$ which activates IDO (indoleamine-2,3-dioxygenase) and subsequently blocks synthesis of tryptophan in RBs [1, 12].

Treatment of RB's with IFN- $\gamma$, Penicillin, or nutrient or iron starvation in cell culture induces a "persistent" non-dividing, but transcriptionally active state. Under these conditions the RBs become enlarged while persisting as an "abberant" $\mathrm{RB}$ that can survive inside the host cell for extended periods of time. The fact that these RB's can be 
recovered from their persistent state and continue their developmental cycle upon removal of the persistence-inducing stimuli hints at possible role for developmental persistence in long-term "silent" chlamydial infections[14].

Decreased organismal load and decreased duration of repeat Chlamydia trachomatis genital infections indicate the induction of adaptive immunity $[9,15,16]$. Interestingly, repeat infections are common and more likely to result in long-term complications indicating that any acquired immunity is incomplete [17]. Host factors such as HLA-DR alleles and IL-10 promoter polymorphisms are also associated with clearance of the pathogen by influencing the ability of the infected persons to mount an effective Th1 immune-response[18].

In a minority of infections, the host immune response induces fibrosis and scarring of the fallopian tubes that are responsible for tubal infertility and ectopic pregnancy $[1,11]$. However, it is still unclear which immune cells are responsible for infection-associated morbidity. Several studies have identified reduced IFN- $\gamma$ production by $\mathrm{CD}^{+}{ }^{+} \mathrm{T}$-cells and Peripheral Blood Mononuclear Cells (PBMC's) as an indicator of tissue damage and long-term sequelae.[19-21].

The challenge of designing a vaccine for immunization against Chlamydia trachomatis would be to initiate a bacteria-clearing Th1-response while avoiding a tissuedestructive inflammatory response in individuals with previous Chlamydia infections[11, 16]. In fact, a dead-whole cell vaccine against ocular Chlamydia trachomatis infections was administered in the 1960 's, which was effective in some individuals, while damaging to those who were re-infected[16]. Furthermore, an ideal vaccine would integrate 
chlamydial antigens that would elicit an effective immune response against all pathogenic Chlamydia species.

\section{Adaptation of pathogenic Chlamydia to their mammalian hosts}

Comparison of ribosomal RNA from Chlamydiae with that of other modern prokaryotes indicates a divergence of Chlamydiae from Proteobacteria and other prokaryotes nearly 2 billion years ago. Pathogenic and environmental species of the order

Chlamydiae are obligate intracellular prokaryotes that split about 700 million years ago. Environmental species reside within unicellular eukaryotes such as free-living amoeba and are ubiquitous in nature. Differences between the genome of the recently characterized Acanthameoba environmental strain UI25 and those of modern pathogenic species have provided insight into the adaptation of pathogenic family Chlamydiaceae to their niche in the mammalian cell.

The genome of the UWE25 strain is more than twice the size $(\sim 2.4$ million $)$ of those of the pathogenic strains ( $\sim$ million $b p)$, while a core set of 711 CDS were conserved among these strains. Adaptation of the pathogenic Chlamydia to mammalian hosts involved a drastic reduction $(\sim 2000 \mathrm{CDS}$ to $1000 \mathrm{CDS})$ in genes, many of which encoded proteins involved in biosynthetic and metabolic pathways. Pathogenic species are auxotrophic for most amino acids and nucleotides and compete with their host cells for them. Other adaptations unique to pathogenic Chlamydia include genes encode possible bacterial adhesins: the major outer membrane protein, MOMP (ompA) and the polymorphic outer membrane proteins (Pmps). These additions to the pathogenic 
chlamydial surface likely play a role in attachment to specific host cell types and/or immune evasion[22].

However, the cysteine-rich outer membrane proteins OmcA and $\mathrm{OmcB}$ that make up Chlamydia's unique disulfide-bonded outer membrane are present in UI25. Unlike other gram-negative bacteria, Chlamydia do not synthesize a peptidoglycan layer on their surface for structure, instead highly crosslinked protein complexes of $\mathrm{OmcA}, \mathrm{OmcB}$, and MOMP provide stability and protect EB's from environmental stress[23]. Conserved genes between U125 and modern pathogenic Chlamydia indicate the importance of their gene products for maintaining intracellular survival. In addition to the outer membrane

proteins $(\mathrm{Omc} A \mathrm{~B})$, these conserved genes encode some inclusion membrane proteins (Incs), components of the type III secretion system (T3SS), as well as some type III secretion effectors.

\section{Type III Secretion}

Type III Secretion Systems are syringe-like molecular machines that are common in pathogenic and non-pathogenic gram-negative bacteria and transport specific bacterial proteins into eukaryotic cells. Pathogenic bacteria often utilize type III secretion systems deliver effectors that hijack cellular functions such as reorganizing the host cytoskeleton, modulating immune signaling or apoptotic factors. A more detailed discussion of type III effector functions will be discussed later. A functional type three secretion system consists of structural components that make up the inner and outer membrane base, the needle shaft, tip, and translocator proteins[24]. It also requires cytoplasmic chaperones 
and a membrane bound ATPase to effectively stabilize and deliver effector proteins, respectively.

These components of Type III Secretion Systems remain conserved across gramnegative species and also share close structural homology with flagellar components. Such structural conservation has allowed for the quick identification of putative chlamydial type III secretion proteins in silico analysis based on conserved structural and genetic domains[25-31]. Many groups have identified putative Chlamydia TTSS components by these approaches, but because of Chlamydia species' genetic intractability, it has remained a challenge to confirm the function of these proteins. Most of our current knowledge of the structure of the chlamydial type III secretion apparatus comes from the discovery of in vitro protein-protein interactions between chlamydial type III components.[27, 32-41], while the detection of new chlamydial effectors has involved expression and secretion of recombinant chlamydial proteins in heterologous type III secretion systems of other bacteria[38, 40-43]. The predicted structure of the Chlamydia type III secretion apparatus and its components is visualized in Figure 1. Assembly of the chlamydial type III apparatus begins with sec-dependent secretion of $\mathrm{CdsC}$, an outer membrane lipoprotein with homology to secretins[44] followed by secretion of CdsJ into the inner membrane[45], and then CdsD into the integral membrane to join the former components into a scaffold that spans two bacterial membranes[31]. CdsD homologs in other bacteria function as scaffolding proteins to link CdsC and other integral membrane components. CdsD has been shown to coimmunoprecipitate with other integral membrane proteins as expected[36], but unique features such as forkhead association domains (FHA) and in vitro phosphorylation are 
puzzling as to the actual role of CdsD in type III secretion[46, 47]. Homology with innermembrane components from Yersinia species indicate that CdsJ provides a scaffold for the inner membrane components $\mathrm{CdsR} / \mathrm{S} / \mathrm{T} / \mathrm{U}$ and V[46]. CdsS and CdsT interact with the C-ring component $\mathrm{CdsQ}$, while the $\mathrm{CdsU}$ homolog in Yersinia $\mathrm{YscU}$ gets cleaved to allow secretion[48]. CdsD has been shown to interact with the cytoplasmic ATPase CdsN and with CdsL, a possible negative regulator of ATPase activity[35]. A complex network of interactions has been shown to occur in vitro between CdsQ, CdsD, CdsN, and CdsL $[35,49]$. Upon assembly of the inner membrane and C-ring components, the machinery becomes secretion competent and secretes its needle subunits CdsF[27], possibly with the help of the putative molecular ruler protein $\mathrm{CdsP}[50]$. Lastly secretion of the tip protein, identified as CT584 in C. trachomatis, completes the latent type III secretion apparatus and is likely to be responsible for activating secretion upon host cell contact[34]. CopN interacts with the ATPase CdsN and prevents secretion of any effectors[41, 51] 


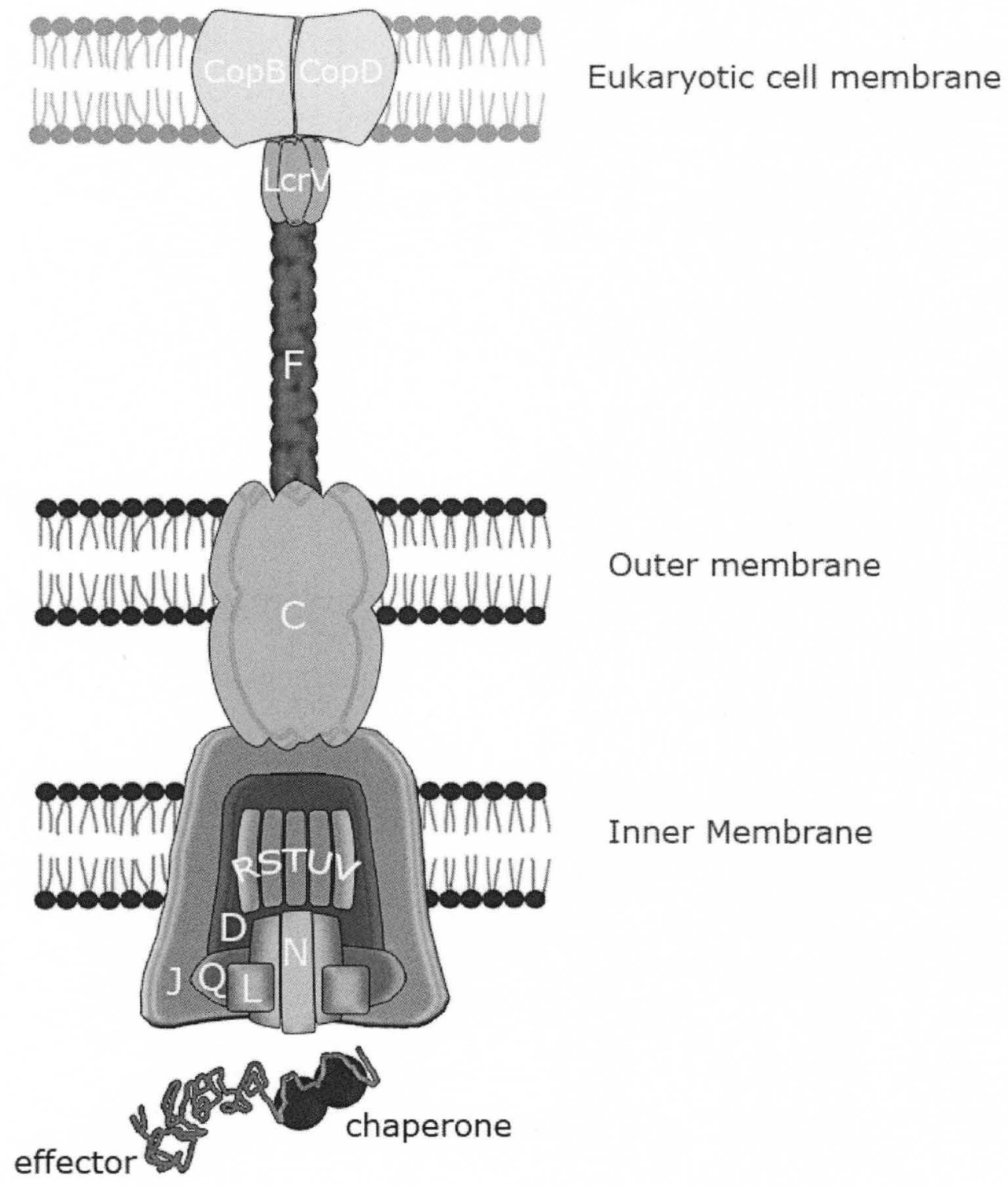

Figure 1. The Type III Secretion System of Chlamydia.

The chlamydial $c d s$ components $C$ - $V$ correspond to their ysc homologues in Yersinia. 
Interaction of the chlamydial EB with the host cell is sensed by the tip protein, which in turn relays an unknown signal to induce CopN secretion. Unblocked, the type III apparatus secretes pairs of translocator proteins (CopB/CopD or $\mathrm{CopB} 2 / \mathrm{CopD} 2)$ to the end of the needle such that they can form a pore in the host cell membrane[37, 38, 46]. After secretion of CopN and the translocator proteins, the ATPase is free to interact with effectors and ensure their translocation into the host cell.

It must be noted however that several of the secreted components and effectors require or utilize molecular chaperones to be efficiently secreted. Bacterial type III secretion chaperones have been organized into classes based on the type of substrates with which they interact. Class I chaperones mediate the translocation of type III effectors and are typically small (13-16 kDa), have an acidic $\mathrm{pI}(4-5)$, and have a tendency to dimerize. CT043, CT088 (also called Scc1), and CT663 have been predicted to be C.trachomatis class I chaperones based on their predicted secondary structure[28, $29,52]$, but to date their interaction with effector proteins has not been confirmed. A recent yeast-2-hybrid screen identified an interaction between CT663 and CT088, indicating formation of a chaperone heterodimer[49]. The same screen also identified a new class Ib chaperone that was capable of interacting with multiple effectors as well with the $\mathrm{C}$-ring component $\mathrm{CdsQ}$ which was described as the multi-cargo secretion chaperone, Mcsc. Characterization of Mcsc by crosslinking also indicated a homodimeric molecular structure. In addition, CT663 has been shown to interact with region 4 of the sigma- 66 subunit of Chlamydia and the $\beta$-subunit of $E$. coli RNA polymerase in a fashion typical of anti-sigma factors[53]. 
The translocator proteins are secreted by Class II chaperones which have tetratricopeptide repeat (TPR) regions. In Chlamydia trachomatis the TPR-containing chapeorones Scc2 and Scc3 are transcribed in operons with their sets of translocators, CopB1/CopD1 and CopB2/CopD2, respectively. Scc2 has been demonstrated to interact with both CopB1 and CopD1, but unexpectedly Scc3 has been shown to interact with CopN, the type III secretion-negative regulator. A third TPR-containing protein, CT274, has been detected in Chlamydia spp. and its small size and acidic pI indicate that it may have chaperone function. Novel interactions between CT274 and the hypothetical proteins CT668 and CT166 indicate possible Chlamydia-specific type III related proteins.

Type III chaperones that are responsible for the stability or secretion or type III components other than effectors or translocators have been classified as Class III chaperones[46]. The needle subunit $\mathrm{CdsF}$ and molecular ruler protein $\mathrm{CdsP}$ have been demonstrated to interact with chaperone products from their respective operons, $c d s E F G$ and $c d s O P$. A yeast-3-hybrid assay indicated that $\mathrm{CdsE}$ and $\mathrm{CdsG}$ form a heterodimer that can interact with $\mathrm{CdsF}[27]$. Multiple studies have identified an interaction between $\mathrm{CdsP}$ and its putative chaperone $\mathrm{CdsO}[33,54]$. Furthermore, $\mathrm{CdsO}$ was shown to form homodimers indicative of type III chaperones. Predicted and experimentally confirmed type III secretion chaperones and their substrates are summarized in Table $\mathbf{1 .}$

Many roles have been reported for the interaction of Class I type III secretion chaperones with their cognate substrates. The most popular role for a Class I chaperone has been to bind approximately the 30-130 N-terminal amino acids of its cognate effector and to unfold the adjacent N-terminal secretion signal. Some chaperones have also been demonstrated to increase the solubility of their substrates or prevent their degradation[55- 
58]. Directly mediating the interaction between the effector and ATPase has also been reported $[59,60]$. Less common functions have included blocking secretion-inhibitory domains or cnzymatic domains of effectors[61, 62].

Many pathogenic bacteria such as Salmonella, E.coli, Shigella, Pseudomonas, and Yersinia species utilize type III secretion systems to export virulence factors into target cells[63]. In these bacteria, the type III secretion-related genes are mostly located in pathogenicity islands or on specific virulence plasmids. However most type III genes in Chlamydia are spread out among ten operons while a few other type III-related genes are completely separate in the genome. In fact this disjointed type III structure is conserved among environmental Chlamydia isolates[64]. Approximately $10 \%$ of the Chlamydia trachomatis serovar D genome is annotated to be type III secretion-related. Considering the small size of the genome it is likely that these are all functional and necessary for productive infection.

In addition to type III secretion components, Chlamydia also contains an incomplete set of flagellar genes. Recent studies have found novel interactions between chlamydial flagellar and type III components. The flagellar ATPase FliI has been shown to have ATP-hydrolyzing activity and interacts in vitro with CdsL and CopN.

Furthermore the flagellar homolog (FlhA) to the inner membrane protein $\mathrm{CdsV}$ can interact with $\mathrm{CdsU}, \mathrm{CdsQ}$, and $\mathrm{CdsL}[32]$. These findings taken together with the fact that Chlamydia is non-motile indicate that the interaction of flagellar components with the type III secretion apparatus may be important in regulating its unique intracellular lifestyle[32, 46]. 


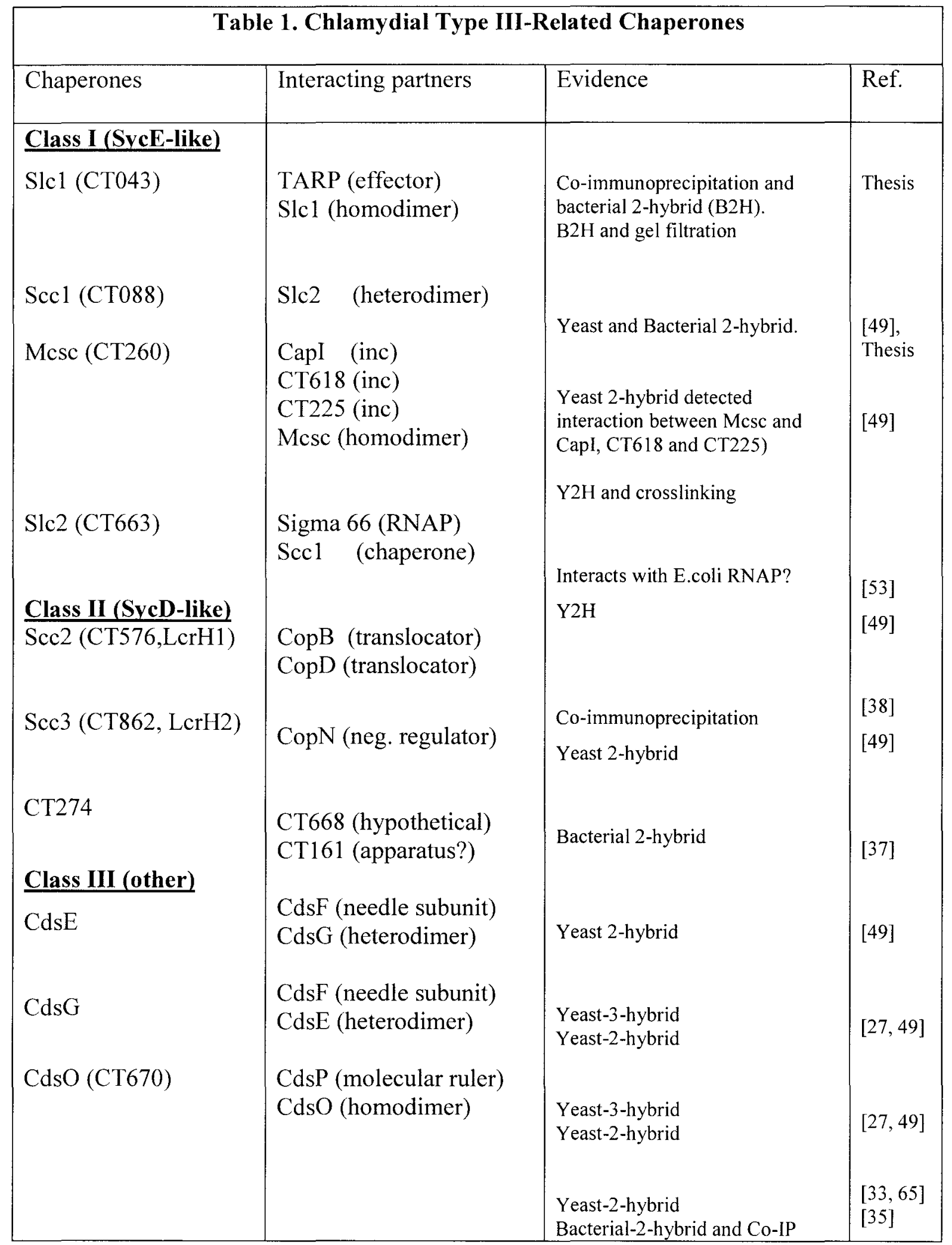




\section{Type III secretion and Chlamydia's developmental cycle}

One common feature of all Chlamydia species is their biphasic developmental life cycle that imposes upon them an obligate intracellular growth restriction. A small noninfectious but metabolically inactive $\mathrm{EB}$, or elementary body, attaches to a eukaryotic cell membrane by reversible electrostatic-interactions with unknown host receptors. A second irreversible interaction occurs and entry into the host cell is guaranteed by the translocation of effectors by a type III secretion apparatus. Pre-formed in the EB and preloaded with virulence factors, the chlamydial type III secretion apparatus translocates its EB-associated effectors into the host cytosol upon contact.

Because of their complex life cycle, it has been challenging for researchers to stably introduce nucleic acids into the bacterium. It is not yet possible to make knockouts, express recombinant proteins or perform small interfering Ribonucleic Acid (siRNA) knockdowns in Chlamydia. For this reason, much of the characterization of chlamydial proteins has focused on their interactions in host cells. Thus the roles of chlamydial type III secreted proteins or effectors in the developmental cycle of Chlamydia have been a major focus of research.

One particular EB-associated effector, TARP, the Translocated Actin $\underline{\text { Recruitment }}$ Phosphoprotein is detected in the host cytosol minutes after infection and is involved in hijacking host signaling molecules to the effect of inducing endocytosis of the attached $\mathrm{EB}[42,66-73]$. Close contact with the eukaryotic cell surface allows interaction of the EB's disulfide-crosslinked outer membrane with host protein disulfide isomerase (PDI) such that the chlamydial outer membrane proteins become reduced and its uptake can occur. Immediately upon uptake into its endocytic vesicle, the EB's DNA starts to 
decondense and it begins to differentiate into a large metabolically active $\mathrm{RB}$ or reticulate body. A small metabolite interrupts the interaction of the chlamydial histone-like proteins $\mathrm{Hc} 1$ and $\mathrm{Hc} 2$ with chromatin and the subsequent decondensation of DNA allows for transcription of early genes.

Expression of inclusion membrane proteins, IncD-G, by RBs is detected within 2 hours of infection[74-76]. Highly divergent in sequence and function, these proteins share large bi-lobal hydrophobic domains of 40-60 amino acids in length that ensure their localization. Secretion and outer membrane localization of these Incs is pivotal to forming its unique intracellular niche, the "inclusion," that diverges from the customary endocytic pathway. IncA, IncG and the Inc CT229 have been demonstrated to be type III secretion substrates by heterologous bacteria, while the mechanism of secretion of other Incs is unknown. Characterization of IncA revealed a eukaryotic SNARE-like domain that may mediate interaction with host vesicles. Similarly IncA has been shown to mediate homotypic fusion of inclusion vesicles[75]. Another host mechanism that Chlamydia mimics to influence the trafficking of host vesicles is the recruitment of Rab GTPases. The Inc CT229 has been shown to interact with Rab4[77], and the $C$.

pneumoniae inc Cpn0585 can interact with Rabs 1, 10 and 11[78]. The Rab6-associating protein Bicaudal- 1 has been found localized to the inclusion as well, possibly by the action of an inc protein[74]. Recruitment of Rab-GTPases to the inclusion can direct vesicles from the golgi, multivesicular bodies, and recycling pathways to the inclusion where essential nutrients, amino acids and specialized lipids can be acquired. Another characteristic of the early inclusion is its recruitment of dynein and subsequent migration toward the host microtubule organizing center (MTOC)[79]. 
A major mechanism of survival of the parasitophorous inclusion is to delay the host cell cycle and preventing apoptosis. The C.trachomatis IncG proteins have been shown to sequester the host protein $14-3-3 \beta$ and prevent phosphorylation of the proapoptosis protein $\mathrm{BAD}[80,81]$. Cleavage of the cell cycle regulator cyclin $\mathrm{B} 1$ was also detected as a consequence of chlamydial infection[82]. The secreted protein CPAF (Chlamydia protease-like secretion factor) has been shown to have several roles during infection including degrading BH3-like apoptosis proteins[83].

Other effectors have been shown to be involved in suppressing host inflammatory signaling. ChlaDub1 and CT441 are able to interrupt NFkB-signalling by deubiquitinating $I \kappa B \alpha$ and by degradation of RelA, respectively[84, 85]. Sequestering of Act1 by the C.pneumoniae effector CP0236 prevents it interaction with IL-17 and subsequent NFkB-activation[86]. CPAF has also been implicated in the degradation of host factors to prevent proper MHC and lipid antigen presentation[75].

As the inclusion becomes modified additional type III secretion needles are assembled and a second set of effectors is expressed. Expression of mid-cycle genes (616 hours p.i.) allows for intermediate metabolism such that RBs can continue to grow and multiply by binary fission. Although Chlamydia do not have a peptidoglycan layer, they are affected by treatment with Penicillin- indicating a possible role for peptidoglycan during division. This theory is corroborated by the transcription of peptidoglycan syntheisis genes prior to cell division and the lack of an FtsZ homolog in Chlamydia species[23]. Possibly by a contact-dependent mechanism, newly divided RBs detach from the inclusion membrane and differentiate back to small infectious EBs. It has been proposed that as RBs divide and the inclusion size increases, individual RBs have less 
surface contact with the inclusion membrane and detachment of the RB occurs, possibly by retraction of type III secretion apparatuses[75, 87, 88]. While microscopic observations and mathematical models support the contact-dependent model of detachment, the mechanism connecting detachment and differentiation remains elusive.

Two distinct mechanisms of EB exit have been identified. Ordered permeabilization of the inclusion membrane, nucleus, and host cell membrane has been demonstrated to release EB's during cysteine protease-induced cell lysis. Extrusion, or a slow pinching off of the chlamydial inclusion, has also been observed at nearly the same frequency as cell lysis during C.trachomatis infection[89]. Future research to define any differences in infectivity, transmission, and pathogenesis by EB's released by lysis versus extrusion will be highly anticipated.

Before secondary differentiation, however, proteins important for invasion, such as bacterial adhesins, components of the type III apparatus and invasion-associated effectors must be expressed and preloaded for immediate translocation into the host cell. Gene expression of type III components have been detected from 8 hours post-infection onward. However, specific subsets of type III-related genes have been shown to be temporally regulated[90-93], indicating functions at different points in the developmental cycle. Evidence of transcription of type III-related genes and their temporal classes are compiled in Table 2. 
Table 2. Expression of type III genes during the chlamydial developmental cycle.

\begin{tabular}{|c|c|c|}
\hline Gene & Temporal class $[39,91-93]$ & Type III function \\
\hline $\operatorname{IncG}$ & E & Effector \\
\hline CT229 (Inc) & E & Effector \\
\hline $\operatorname{Inc} A$ & M & Effector \\
\hline TARP & $\mathrm{M}, \mathrm{VL}$ & Effector \\
\hline Slc 1 & M & Chaperone (I) \\
\hline Sccl & $\mathrm{C}$ & Chaperone (I) \\
\hline Slc2 & M & Chaperone (I) \\
\hline $\operatorname{FliF}(\mathrm{J})$ & M & Apparatus \\
\hline FliN (Q) & $\mathrm{L}$ & Apparatus \\
\hline FliA & M & Sigma 28 \\
\hline FlhA (V) & M & Apparatus \\
\hline Flil $(\mathrm{N})$ & $\mathrm{L}$ & ATPase \\
\hline $\mathrm{CdsC}$ & $\mathrm{L}$ & Apparatus \\
\hline CdsE & M & Apparatus \\
\hline CdsL & M & Apparatus \\
\hline CdsJ & M & Apparatus \\
\hline CdsR & $\mathrm{M}, \mathrm{C}$ & Apparatus \\
\hline $\mathrm{CdsS}$ & $\mathrm{M}, \mathrm{C}$ & Apparatus \\
\hline CdsT & $\mathrm{L}, \mathrm{C}$ & Apparatus \\
\hline $\mathrm{Cds} \mathrm{U}$ & $\mathrm{M}, \mathrm{C}$ & Apparatus \\
\hline $\mathrm{CdsV}$ & $M, L$ & Tip /Cap \\
\hline CT274 & M & Chaperone (II) \\
\hline $\mathrm{Scc} 2$ & $\mathrm{~L}$ & Chaperone (II) \\
\hline $\operatorname{Scc} 3$ & M & Chaperone (II) \\
\hline CopB & $\mathrm{L}$ & Translocator \\
\hline CopD & $\mathrm{L}$ & Translocator \\
\hline CopB2 & M & Translocator \\
\hline CopD2 & M & Translocator \\
\hline CopN & $\mathrm{M}$ & Neg. Regulator \\
\hline
\end{tabular}

1. $\mathrm{E}=$ early, $\mathrm{M}=$ Mid-cycle, $\mathrm{L}=$ Late-cycle, $\mathrm{VL}=$ Very Late $\mathrm{C}=$ constituitively active.

2. Type-III apparatus homolog to Flagellar proteins is in parenthesis. 


\section{Attachment}

The initial association of Chlamydia elementary bodies to the epithelial cell surface has been shown to involve a heparin-sensitive reversible electrostatic interaction with host cells. A plethora of data has been accumulated regarding the role of heparansulfate like glycosaminoglycans (GAGs) in this attachment step. Early studies to determine possible bacterial adhesins demonstrated that infection can be limited by pretreatment of the bacteria or host cells with heparan-sulfate[94-101]. Coating of Chlamydia trachomatis and pneumonaie EBs with heparan-sulfate specific monoclonal antibody prevents infection[97]. Treatment of host cells or EBs with heparinase or with chemically-modified heparin-derivatives also prevented infection of C. trachomatis serovar E and L2 strains[99, 101]. Several groups have attempted to determine the role of host cell heparan sulfate in attachment by infecting heparan-sulfate deficient mutant $\mathrm{CHO}$ cell lines. A few of these studies have indicated a reduction in attached EBs to these cell lines, while a more recent study has indicated that host heparan-sulfate is not necessary for attachment.

Substantial evidence implicates the chlamydial antigen MOMP (major outer membrane protein) in the initial binding of host cells. It was shown that trypsin-mediated cleavage of the variable regions of MOMP inhibited C.trachomatis serovar B, but not L2 infection $[102,103]$. Furthermore, in vitro treatment of host cells with MOMP-specific antibodies severely abrogates $C$. trachomatis EB binding and infection. EB-surface associated MOMP is linked to a glucosamine containing sulphated-polysaccharide that seems to be important for binding mammalian cells[96, 104-107]. N-glycanase cleavage of this polysaccharide prevents infection by C. trachomatis strains $[105,106,108]$. This 
MOMP-associated GAG has likely complicated the interpretation of the heparin, heparan-sulfate, and heparinase experiments mentioned earlier.

A second temperature-dependent binding step was discovered by infection of a mutant $\mathrm{CHO}$ cell line with C.trachomatis L2 strain[109]. Attachment of EBs to wild-type CHO cells at $4^{\circ} \mathrm{C}$ could be reversed by addition of heparin, but attachment at $37^{\circ} \mathrm{C}$ could not. Isolation of a mutant cell line that could be competed for binding to EBs by heparin at $37^{\circ} \mathrm{C}$ indicated a loss of the second heparin-resistant, temperature-dependent receptor.

Many different host ligands have been proposed as this second receptor that remains irreversibly bound to EBs. Mannose-6-Phosphate treatment of epithelial cells can inhibit attachment and entry of C. pneumoniae, indicating a possible role for Mannose-6-Phosphate receptor/Insulin Growth Factor 2 as a receptor[110]. Knockdown of host cell Platelet-derived growth factor receptor- $\beta$ (PDGFR $\beta$ ) with siRNA and treatment with PDGR $\beta$-monoclonal antibody reduce EB binding[111]. PDFR $\beta$ has also been indicated as an important player in Chlamydia pneumoniae infection by a recent systems biology study[112]. A recent study of the role of the cellular cystic fibrosis transmembrane conductance regulator (CFTR) during C. trachomatis infection describes CFTR-dependent uptake of EBs and an increase in EB internalization by CFTRectopically expressing cells[113].

Other bacterial adhesins besides MOMP that have been implicated in host receptor binding include LPS, the outer membrane complex protein $\mathrm{OmcB}$, and the polymorphic membrane proteins Pmp6, Pmp20, and Pmp21. OmcB from C.trachomatis serovars LGV1 and E show GAG-dependent and independent binding to host cells, respectively, indicating that it may be involved in either the primary or secondary 
attachment step[114]. C. pneumoniae Pmp proteins contain tetrapeptide repeat motifs that mediate binding to epithelial cells, and a recent study indicates that the presence of multiple Pmps have an additive effect on attachment to host cells[115].

A complex role for Protein Disulfide Isomerase (PDI) in attachment was also discovered. Reduced attachment of C.trachomatis to a CHO cell line with a mutatedleader sequence in PDI could be restored by complementation with the full length PDI[116]. This requirement of PDI for attachment has been confirmed in all other pathogenic Chlamydia. However, it is suspected that PDI does not behave as an actual receptor but instead structurally associates with another host receptor to initiate attachment[117].

While enzymatic activity of host PDI is not necessary for attachment, it is an absolute requirement for chlamydial entry into epithelial cells. Thus, as the chlamydial EB is brought in close vicinity of the eukaryotic membrane by electrostatic interactions, another secondary host receptor in coordination with PDI irreversibly binds and reduces the crosslinked-outer membrane proteins and initiates uptake events such as the activation of the type III secretion system. Tight-binding of the type III secretion apparatus to the host cell membrane could indicate that the type III needle and tip proteins as possible secondary receptors, though further research in this area is needed to corroborate or disprove the role of the type III secretion apparatus in attachment.

Despite years of research dedicated to the search for host and chlamydial receptors that mediate attachment, the use of a variety of host cell types and chlamydial species has made it difficult to determine a general mechanism for attachment. A compendium of this work can be found in Tables 3 and 4 . 


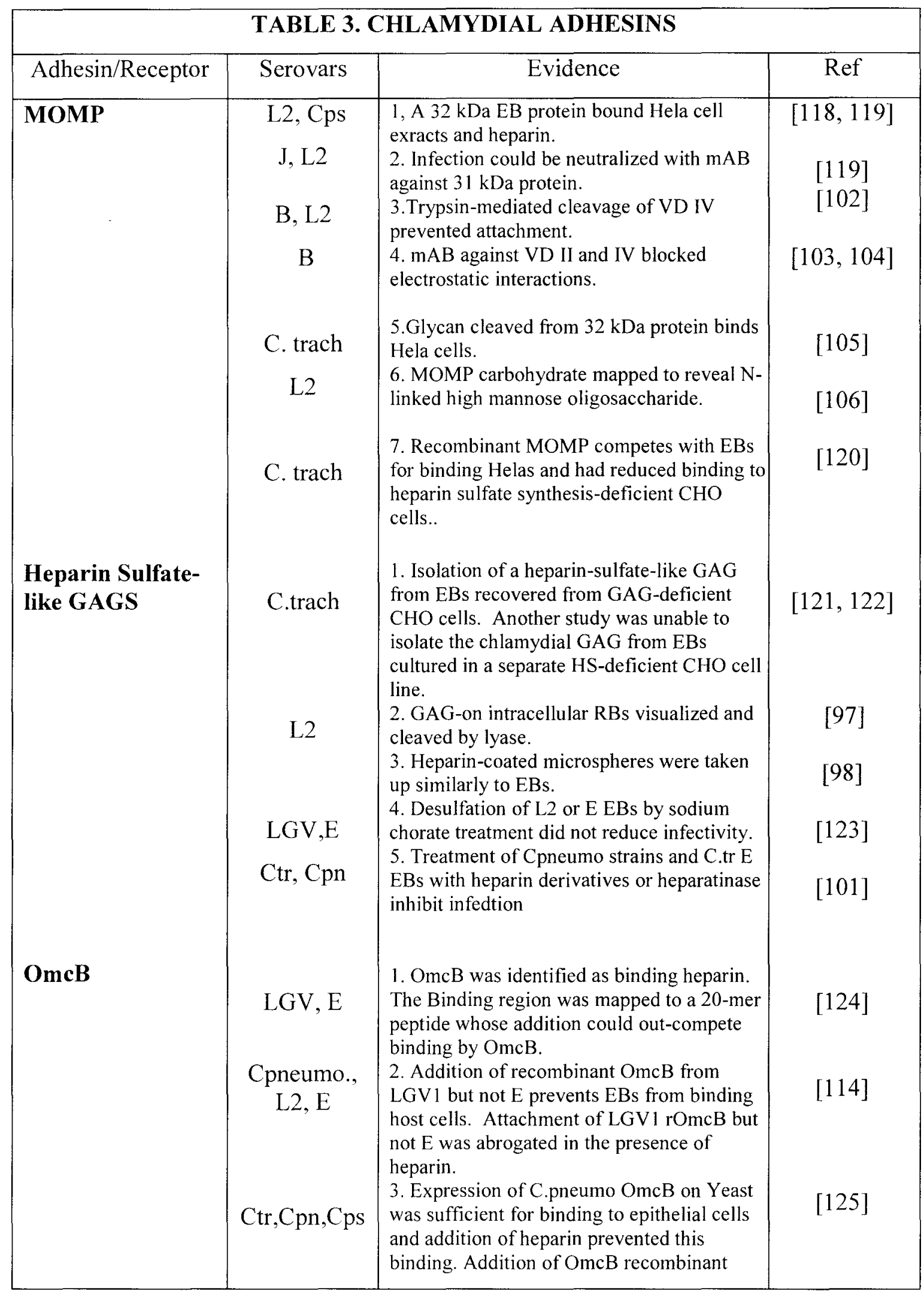




\begin{tabular}{|l|l|l|c|}
\hline LPS & E, LGV & $\begin{array}{l}\text { protein or monoclonal antibody prevents } \\
\text { C.pneumo EB attachment to host cell. } \\
\text { 1.Chalmydia LPS immunoprecipitates with } \\
\text { CFTR. }\end{array}$ & [113] \\
Pmps & Cpn, L1 E & $\begin{array}{l}\text { 1. Recombinant expression of Pmp6, 20, or } \\
\text { 11 on yeast cells mediates adhesion to } \\
\text { epithelial cells. Treatment of host cells with } \\
\text { recombinant Pmps or mAb against these } \\
\text { proteins prevents C pneumo infection. } \\
\text { Binding can be attributed to the tetrapeptide } \\
\text { motifs in these proteins. }\end{array}$ & {$[115]$} \\
\hline
\end{tabular}

\section{TABLE 4. HOST CELL RECEPTORS}

\begin{tabular}{|c|c|c|c|}
\hline Receptor & Cell types & Evidence & Ref \\
\hline PDGFR $\beta$ & S2, Hela & $\begin{array}{l}\text { 1. Treatment of host cells with PDGFR } \beta \text { siRNA or } \\
\text { mAb reduces C.trachomatis } \mathrm{L} 2 \text { binding. }\end{array}$ & {$[111]$} \\
\hline CFTR & $\begin{array}{l}\text { Hela } \\
\text { CFTR-/- mice }\end{array}$ & $\begin{array}{l}\text { 2. Uptake of C.trachomatis EBs is blocked by } \\
\text { CFTR mAbs and by treatment with CFTR } \\
\text { inhibitors. Also there was a reduction in EB } \\
\text { uptake by CFTR knockout mice. CFTR can also } \\
\text { be co-immunoprecipitated with LPS. }\end{array}$ & [113] \\
\hline M6P receptor & HMEC-1 & $\begin{array}{l}\text { 1. Mannose-binding protein interacts with MOMP } \\
\text { and prevents interacation of C.trachomatis with } \\
\text { host cells. } \\
\text { 2. Addition of M6PR or M6P analog prevents } \\
\text { attachment of C.pneumo but not C.trachomatis } \\
\text { strains to endothelial cells. }\end{array}$ & {$[110]$} \\
\hline PDI & CHO-6, Hela & $\begin{array}{l}\text { 1. The CHO- } 6 \text { cell line is mutant for PDI and is } \\
\text { resistant to attachment that could be recovered } \\
\text { with FL PDI. } \\
\text { 2. siRNA knockdown of PDI prevents infection. } \\
\text { Chlamydia does not bind directly to PDI-gpi. }\end{array}$ & {$[117]$} \\
\hline
\end{tabular}




\begin{tabular}{|c|c|c|c|}
\hline \multicolumn{4}{|c|}{ TABLE 4. HOST CELL RECEPTORS (cont.) } \\
\hline Receptor & Cell types & Evidence & Ref \\
\hline $\begin{array}{l}\text { Heparin- } \\
\text { sulfate }\end{array}$ & $\begin{array}{c}\text { Hela } \\
\text { Hela, CHO761 } \\
\text { L929, Hela } \\
\text { Hela, L929 } \\
\text { CHO } \\
\text { L929,Hela } \\
\text { CHO } \\
\text { Hela, McCoy } \\
\text { Hela, McCoy } \\
\text { Hela } \\
\text { Hela, CHO } \\
\text { McCoy, Hela } \\
\text { CHO }\end{array}$ & $\begin{array}{l}\text { 1. } 32 \text { kDa and } 18 \text { kDa EB surface proteins are } \\
\text { prevented from binding host cell by heparin. } \\
\text { 2. Infection inhibited by adding heparin-sulfate } \\
\text { receptor or heparatinase. } \\
\text { 3. Heparatinase treatment of LGV and trachoma } \\
\text { EBs prevented attachment. } \\
\text { 4. Heparin inhibition of LGV and trachoma biovar } \\
\text { infections was rescued by competition with a } \\
\text { sulphated decasaccharide. } \\
\text { 5. A heparin sulfate synthesis-deficient CHO cell } \\
\text { line had reduced EB infectivity. A separate HS- } \\
\text { deficient CHO cell line was resisitant LGV but not } \\
\text { E infection. } \\
\text { 6. Attachment of LGV EBs to was completely } \\
\text { inhibited by pretreatment with heparin or } \\
\text { heparatinase. } \\
\text { 7. Attachment of Trachoma biovar EBs was only } \\
\text { partially inhibted by heparin or heparatinase } \\
\text { pretreatment. } \\
\text { 8. Serovar E attachment not inhibited } \\
\text { by heparin or heparan sulfate, but L2 was } \\
\text { completely inhibited. } \\
\text { 9. Heparatinase blocked C.psittaci EB attachment } \\
\text { at } 4^{\circ} \text { and } 37^{\circ} \text { while heparin blocked attachment at } \\
4 \text {. } \\
\text { 10. Inhibition of C.trachomatis infectivity by } \\
\text { sulphated polymers or polysaccharides in vitro (cell } \\
\text { culture) but not in vivo (mouse model) } \\
\text { 11. Heparin sulfate mAb binds to C. trach and C.pn } \\
\text { EB surface and neutralizes infectivity. } \\
\text { 12. Heparatinase treatment of host cells prevented } \\
\text { L2 infection and the HS-synthesis deficient CHO } \\
\text { cell line was less sensitive to infection. } \\
\text { 13. Desulfation of host cells by sodium chlorate } \\
\text { inhibited L2 but not E infection. } \\
\text { 14. The HS mutant cell line CHO-18.4 was } \\
\text { infected equally by L2 and D and was comparable } \\
\text { to infection of WT CHO cells. } \\
\text { 15.Treatment of host cells with heparinase and } \\
\text { heparin derivatives prevented infection by C.pn } \\
\text { strains and C.tr E. } \\
\text { 16. OmcB-expressing yeast from Cpn and C.tr-L1 } \\
\text { were unable to bind heparatinase treated or GAG- } \\
\text { deficient CHO cells. }\end{array}$ & $\begin{array}{l}{[97]} \\
{[99]} \\
{[123]} \\
{[100]} \\
{[101]} \\
{[125]}\end{array}$ \\
\hline
\end{tabular}




\section{Entry}

Following attachment of EBs, uptake by the endocytic pathway is induced by chlamydial factors. Uptake of chlamydial EBs by epithelial cells is associated with the recruitment of actin and tyrosine phosphorylation of proteins at the site of entry for C.trachomatis $[42,73,129,130]$. This actin recruitment is dependent on the activation of the small Rho GTPase Rac in C.trachomatis infection, while both Cdc42 and Rac activation are important for C.caviae entry[72, 131]. A tyrosine-phosphorylated protein associated with actin recruitment has been identified to be the chlamydial type III translocated protein TARP. C.trachomatis LGV TARP contains six tyrosine-rich repeat domains that can be tyrosine phosphorylated by several host kinases containing the Srchomology 2 -domain (SH2). The SH2-containing kinases Src, Yes, Fyn, Syk, and Abl are all able to phosphorylate tyrosine residues of C.trachomatis TARP $[66,68,132]$. The observation that individual knockdown of any one of these kinases does not completely abrogate tyrosine phosphorylation reinforces the importance of TARP phosphorylation for efficient manipulation of host signaling cascades and actin remodeling. Type III effector-induced actin polymerization and cytoskeletal rearrangement has been extensively studied in other bacteria pathogens. Enteropathogenic E.coli stimulates acitnpedestal formation through the type III translocation of Tir (translocated intimin receptor) into host cells where it is tyrosine-phosphorylated and associated with Arp2/3 recruitment[133, 134]. The SPI-1 (Salmonella Pathogenicity Island-1) type III secretion system induces bacterial uptake by translocation of effectors that induce membrane ruffling and Rho GTPase activation[135]. 
Tyrosine phosphorylated residues in C.trachomatis TARP are able to recruit the guanine nucleotide exchange factors Sos-1 and Vav-2[67, 68]. A complex of Sos-1, Eps8, and Abi-1 or Vav-2 with PIP3 (phospho-inositol 3,4,5- $\mathrm{P}_{3}$ ) have been shown to activate Rac in a phosphotyrosine dependent manner[67]. Rac-dependent recruitment of actin has been shown to involve Abi-1 and WAVE-2 dependent formation of the actinnucleating Arp 2/3 complex[69]. While C.trachomatis L2 and D TARPs contain 6 and 3 tyrosine-rich repeats (TRR), respectively, C.caviae, C.pneumoniae, and C.psittaci do not contain TRRs. All chlamydial TARPs do contain proline-rich domains that induce TARP aggregation and C-terminal WH2-like domains that are capable of nucleating actin [70]. It has been proposed that the WH2 domains on TARP may contribute to the further polymerization of the short actin filaments already induced by the Arp2/3 complex[69].

A recent protein microarray indicated the interaction of an SH2-containing adaptor protein SHC1 with TARP derived phosphopeptides. Infection of SHC1 knockdown cells sensitized Chlamydia to TNF-induced apoptosis, and detection of SHCl-regulated genes included those involved in apoptosis and growth[136]. Taken together, previous findings clearly indicate that TARP plays a critical role in the entry and survival of Chlamydia trachomatis in host cells, and thus its translocation through the bacterial type III secretion apparatus into mammalian cells must be absolutely efficient to ensure survival of the organism.

Besides TARP, the EB-associated type III effectors CT166 and CT694 have been reported to be associated with entry-related signaling. As a homolog to the chlostridial glucosylating toxins, the mono-glucosylation and subsequent deactivation of Rac by CT166 has been demonstrated in Hela cells. Since this effector is translocated into host 
cells shortly after infection it is likely that it provides a balance to the actin remodeling induced by TARP during C.trachomatis entry[137]. The association of CT694 with the host protein AHNAK to alter stress fibers may also be important for invasion.[138]

Tyrosine-phosphorylation of the host protein Ezrin has also been discovered as a direct result of C.trachomatis infection of epithelial cells[139]. Like TARP, the tyrosinephosphorylation of Ezrin upon infection led to its association with actin, specifically in microvilli.[140] The specific kinase(s) involved in Ezrin tyrosine phosphorylation during Chlamydia infection have not been determined, but previous studies have demonstrated that this event can be a direct result of host receptor binding and activation[141].

Chlamydial attachment to and subsequent phosphorylation of PDGFR $\beta$ results in phosphorylation of WAVE2, Cortactin, and the guanine-exchange factor Vav2[111]. Phosphorylation and subsequent activation of these molecules is associated with actinremodeling events. It is clear that multiple and redundant signaling pathways are hijacked by Chlamydia species to ensure efficient uptake of the organism. Upon cell contact, immediate secretion of chlamydial factors such as TARP into the host cytosol is critical for initiating actin-cytoskeletal remodeling and subsequent endocytosis of the EB. 


\section{HYPOTHESIS}

I hypothesize that a chlamydial factor is responsible for the efficient association of TARP with the type III secretion apparatus and its ensuing translocation into the host cell cytosol. I propose that the Chlamydia trachomatis Class I type III secretion chaperone Slc1 interacts with TARP and is responsible for its efficient translocation through the type III apparatus. 


\section{CHAPTER II.}

\section{MATERIALS AND METHODS}

\section{Bacterial strains and cell culture.}

Chemically competent Top 10 and BL21star E. coli strains from Invitrogen were used for plasmid propagation and protein expression, respectively. The adenylate cyclase (cya) deficient DHM1 E. coli strains were grown at $30^{\circ} \mathrm{C}$ on indicator plates (LB $+40 \mu \mathrm{g} / \mathrm{ml}$ $\mathrm{X}$-gal $+1 \mathrm{mM}$ IPTG or McConkey $+1 \%$ maltose) for 48 hours or in Luria-Bertani broth (LB) at $37^{\circ} \mathrm{C}$. All other E. coli strains were grown at $37^{\circ} \mathrm{C}$ in $\mathrm{LB}$ broth or agar plates. The Yersinia enterocolitica WT strain MRS40 or $\Delta$ YscN E40(pMS141) strain were grown in LB or Brain-Heart-Infusion (BHI) broth at $26^{\circ} \mathrm{C}$. Media was supplemented with $100 \mu \mathrm{g} / \mathrm{ml}$ carbenicillin (carb), $60 \mu \mathrm{g} / \mathrm{ml}$ kanamycin (kan), $12.5 \mu \mathrm{g} / \mathrm{ml}$ tetracycline (tet), or $35 \mu \mathrm{g} / \mathrm{ml}$ Nalidixic Acid, when appropriate. The wild-type EPEC serotype O127:H6 strain E234/69 and the cesT knockout strain were obtained from Gadi Frankel's lab and grown in LB broth. The Salmonella eneterica typhimurium strain $12023 \mathrm{~s}$ was obtained from David Holden's lab and grown in LB broth. Hela cells were cultivated at $37^{\circ} \mathrm{C}$ with $5 \% \mathrm{CO}_{2}$ in DMEM supplementedwith $10 \% \mathrm{FBS}$ and $2 \mathrm{mM} \mathrm{L-glutamine.}$

\section{Plasmid construction.}


Slc1, Scc1, Mcsc, Slc2, and the N-terminal 600 nucleotides of TARP were amplified from Chlamydia trachomatis genomic DNA (gDNA), CesT and the N-terminal $600 \mathrm{nt}$ of Tir from enteropathogenic E. coli gDNA, and YopH from Y.enterocolitica MRS40 gDNA by the polymerase chain reaction (PCR) using the primers listed in Table 5. Cloning into pET101, pET200, pBAD-TOPO and pENTR-D/SD-TOPO utilized the endogenous topoisomerase activity of Invitrogen directional cloning vectors. The PCR fragments CACC-CT043-FLAG-stop (and CACC-CT663-FLAG-stop were cloned into pET101/D-TOPO, resulting in the plasmids pET101-Slc1-FLAG and pET101-Slc2FLAG, respectively. The PCR fragment CACC-TARP ${ }^{1-200}$ was cloned into pET200/DTOPO, resulting in the plasmid pET200-TARP ${ }^{1-200}$. All of the constructed plasmids were transformed into Top 10 cells, recovered in SOC medium for one hour at $37^{\circ} \mathrm{C}$, and plated on LB agar supplemented with carb, kan or both. The resulting colonies were restreaked and grown overnight. Presence of the correct plasmid inserts was confirmed by colony PCR and sequencing. The PCR fragments CACC-CT043 and CACC-CT088FLAG were cloned into pENTR/SD/D-TOPO, resulting in the plasmids pENTR-Slc1 and pENTR-Scc1-FLAG, respectively. These new entry vectors were recombined with pET56-DEST using LR recombinase II reaction mix (Invitrogen), transformed into Top10, and selected for on $\mathrm{LB}+\mathrm{carb}$ agar overnight. The resulting colonies were restreaked on carb plates and grown overnight. The presence of the correct plasmids was determined by colony PCR and sequencing. Plasmid DNA was purified from the Top10 genetically modified strains by using Qiaprep Spin Miniprep Kit.(Qiagen). The BL21star expression strain was singly or co-transformed with pET200-TARP200, pET101-Slc1, pET101-Slc2, and pET56-Scc1-FLAG. For the expression of chlamydial proteins in Yersinia, I digested 
Slc2 and Scc1 from pet101-Slc2 and pet101-Scc1 with Xbal and ScaI-HF (NEB). These digested inserts were then ligated into the Xbal and ScaI sites of pBAD18 (American Tissue Culture Collection). Mcsc and Slc1 were cloned into the pBAD-TOPO vector. SLIM PCR was used to add a KpnI sited downstream of TARP in the vector pet200-

$\mathrm{TARP}^{1-200}$ to form pet200-TARP $\mathrm{P}^{1-200}-\mathrm{kpnI}$. This vector was then digested with NdeI and $\mathrm{KpnI}$ and the resulting NdeI-TARP ${ }^{1-200}-\mathrm{KpnI}$ fragment was cloned into these sites in pCX340. For co-expression in the bacterial 2-hybrid system, all of the PCR inserts were generated to have flanking $\mathrm{XbaI}$ and $\mathrm{KpnI}$ sites and were cloned into the same sites in pUT18C and/or pKT25 prior to co-electroporation into DHM1 E. coli.

\section{Protein expression, lysate preparation, and protein purification.}

Overnight cultures of BL21star strains were diluted 1:20 in LB + antibiotic, and grown to $\mathrm{OD}_{600}$ of 0.6 . Expression of recombinant proteins was induced by addition of $0.5 \mathrm{mM}$ isopropyl- $\beta-\mathrm{D}$ thiogalactopyranoside (IPTG) and incubated for 3 hours with shaking. Bacteria were centrifuged at $8000 \mathrm{~g}$ for 20 minutes, and the pellet was resuspended in $1 / 10$ culture volume of lysis buffer $\left(50 \mathrm{mM} \mathrm{NaH}_{2} \mathrm{PO}_{4}, 300 \mathrm{mM} \mathrm{NaCl}, 0.05 \%\right.$ TIen, 0.1 mg/ml Dnase I, 1 mg/ml lysozyme, Halt Protease Inhibitor Cocktail (Pierce)). For preparation of lysates that were added to NiNta Magnetic Beads, $25 \mathrm{mM}$ imidazole was added to the lysis buffer. Bacteria were incubated in lysis buffer for 30 minutes at room temperature, sonicated to shear DNA, and stored at $-80^{\circ} \mathrm{C}$. Purifications of Sic1-FLAG and His6- TARP $\mathrm{P}^{1-200}$ for use in crosslinking and gel filtration experiments were performed using M2-Anti-FLAG agarose (Sigma) and Ni-Nta Agarose (Qiagen), respecitively. Lysozyme was left out of the lysis buffer and the french press was used to 
release soluble proteins from these lysates prior to clearing by centrifugation at $15,000 \mathrm{~g}$ for 30 minutes. Purification fractions were run on 12\% SDS-PAGE gels and proteins were stained with a $0.2 \%$ Coomassie Blue R250 solution.

\section{Coimmunoprecipitation assays and immunodetection.}

Bacterial expression lysates were thawed and partially cleared by centrifugation at $4000 \mathrm{~g}$ for 15 minutes prior to addition to M2 Anti-FLAG Agarose (Sigma) or NiNta Magnetic Beads (Qiagen). $30 \mathrm{mM}$ imidazole was added to $500 \mu \mathrm{l}$ lysates prior to incubation with the magnetic beads on an end-over-end rotator overnight at $4^{\circ} \mathrm{C}$. The flow-through fraction or supernatant was separated from the beads with a magnetic separator, and the beads were washed five times with NiNta Native Wash Buffer ( $50 \mathrm{mM} \mathrm{Na}_{2} \mathrm{HPO} 4,300$ $\mathrm{mM} \mathrm{NaCl}, 20 \mathrm{mM}$ imidazole, $0.05 \%$ TIen 20, PH 8.0). The first (W1) and fifth (FW) wash fractions were kept for analysis, and the native protein complexes were eluted from the beads by incubation with $100 \mu \mathrm{l}$ NiNta Native Elution Buffer ( $50 \mathrm{mM} \mathrm{Na} 2 \mathrm{HPO} 4,300$ $\mathrm{mM} \mathrm{NaCl}, 300 \mathrm{mM}$ imidazole, $0.05 \%$ Tlen $20, \mathrm{pH} 8.0$ ) at $4^{\circ} \mathrm{C}$ for 4 hours. For each coimmunoprecipitation experiment with M2 Anti-FLAG agarose, $1 \mathrm{ml}$ of bacterial lysate was added to $100 \mu \mathrm{l}$ of packed agarose resin and incubated on an end-over-end rotator at $4^{\circ} \mathrm{C}$ overnight. The flow-through fractions were collected by centrifugation at $3000 \mathrm{~g}$ for 1 minute, and the resin was washed five times with tris-buffered saline (TBS, prior to elution of the native protein complexes by incubation with $100 \mu \mathrm{l}$ of $200 \mathrm{ng} / \mathrm{ml} 3$ XFLAG peptide (Sigma) in TBS at $4^{\circ} \mathrm{C}$ for 4 hours-overnight. Immunoprecipitations of S-tagged proteins were done as per the S-tag ReK purification kit manual. (Novagen). Immunoprecipitation fractions were incubated 1:1 with Laemmli sample buffer (Biorad) 
$+0.5 \% \beta$-mercaptoethanol at $95^{\circ} \mathrm{C}$ for 10 minutes prior to separation by SDS-PAGE. Proteins were transferred to polyvinylidine fluoride (PVDF) membrane and detected by Western blot probed with 6XHIS, DDDDK, and S-tag specific antibodies from Abcam (cat\#: ab18588, ab21536, ab18184, ab49763, ab1187). HRP-conjugated goat-anti-mouse (Millipore 12-349) or HRP-goat-anti-rabbit (Pierce \#185415) were used as secondary antibodies when necessary, followed by development with Millipore Immobilin Western Substrate.

\section{$\beta$-galactosidase assay.}

A modified Miller assay was followed[142]. Briefly, co-expressing DHM1 strains were grown overnight in $\mathrm{LB}+$ antibiotic at $37^{\circ} \mathrm{C}$ followed by a $1: 50$ subculture and growth in fresh LB+antibiotic to an OD600 of $0.5-0.8$. The OD600 of the culture was recoded and $20 \mathrm{ul}$ of culture was added to $80 \mathrm{ul}$ of permeabilization buffer $(20 \mathrm{mM} \mathrm{KCl}, 2 \mathrm{mM}$ $\mathrm{MgSO}_{4}, 0.8 \mathrm{mg} / \mathrm{mL}$ CTAB (hexadecyltrimethylammonium bromide), $0.4 \mathrm{mg} / \mathrm{mL}$ sodium deoxycholate, $5.4 \mu \mathrm{L} / \mathrm{mL}$ beta-mercaptoethanol) and incubated at $30^{\circ} \mathrm{C}$ for 30 minutes. The time was recorded as $600 \mathrm{ul}$ of pre-warmed substrate solution $\left(60 \mathrm{mM} \mathrm{Na}_{2} \mathrm{HPO}_{4}, 40\right.$ $\mathrm{mM} \mathrm{NaH}{ }_{2} \mathrm{PO}_{4}, 1 \mathrm{mg} / \mathrm{mL}$ o-nitrophenyl- $\beta$-D-Galactoside (ONPG), $2.7 \mu \mathrm{L} / \mathrm{mL} \beta$ mercaptoethanol) was added to each permeabilized sample and incubated at $30^{\circ} \mathrm{C}$. Upon developing a yellow color, the samples were stopped with $700 \mathrm{ul}$ of $1 \mathrm{M}$ Sodium Carbonate $\left(\mathrm{Na}_{2} \mathrm{CO}_{3}\right)$ and the stop time was noted.

\section{Colocalization assay.}


The open reading frames of Slc1 and Slc2 were cloned into pENTR/D-TOPO and recombined into pDest-N-mcherry by the LR recombinase reaction as described by the Gateway expression manual. (Invitrogen) Mcherry-Slc1, Mcherry-Slc2, or Mcherry gus were co-transfected with full length C. caviae GFP-TARP into Cos-7 cells with Fugene 6 Transfection Reagent as indicated in the Fugene 6 Technical Bulletin. (Roche)

Transfected constructs were expressed for $24 \mathrm{hrs}$ before the cells were fixed with $4 \%$ Paraformaldehyde on glass coverslips and analyzed with an Olympus Fluoview 500 Laser Scanning Microscope.

\section{Secretion Assay.}

Overnight cultures of Y.enterocolitica strains containing pcx340 and pBAD vectors were diluted to an OD600 of 0.2 in $5 \mathrm{ml}$ of $\mathrm{BHI}+2.5 \mathrm{mM} \mathrm{CaCl} 2$ (represses Yop production) or $\mathrm{BHI}+0.4 \%$ glucose $+5 \mathrm{mM} \mathrm{EGTA}+20 \mathrm{mM} \mathrm{MgCl} 2$ (induces Yop production) for 2 hours at $26^{\circ} \mathrm{C}$. Expression of recombinant proteins were induced with the addition of 1 $\mathrm{mM}$ IPTG and $13 \mathrm{mM} \mathrm{L}$-Arabinose, upon shifting to $37^{\circ} \mathrm{C}$ (type III secretion-inducing conditions). Secretion was induced for 4 hours prior to centrifugation at $3000 \mathrm{~g}$ for 20 $\min$. The top $9 \mathrm{ml}$ of the supernatant were removed and respun at $12000 \mathrm{~g}$ for 30 minutes at $4^{\circ} \mathrm{C}$. This supernatant was further cleared with a $0.45 \mathrm{uM}$ filter and proteins were precipitated with ice-cold 10\% TCA. Precipitated proteins were pelleted, washed twice with ice-cold acetone, and dried prior to resuspension in a proportional volume of Lysis buffer B (0.1 M Tris-Hcl, 0.1 M NaH2PO4, $300 \mathrm{mM} \mathrm{NaCl,} 6 \mathrm{M}$ Urea) to cell pellet. Lysates were treated 1:1 with Laemmli $+5 \% \beta \mathrm{ME}$ and heated to $95^{\circ} \mathrm{C}$ for 15 minutes priort to separation on a $12 \%$ SDS-PAGE gel. Protein concentration in the whole-cell 
pellet was determined by bicinchoninic acid assay (BCA) and the protein in whole-cell pellets was normalized to $1 \mathrm{mg} / \mathrm{ml}$ in Lysis buffer B.

\section{Yersinia enterocolitica Translocation Assay.}

Hela cells were plated in black, flat, glass-bottomed 96-Ill plates at a density of 1 X 104 cells per III and alloId to grow for 24 hours prior to infection. One day prior to infection TEM-1 fusion protein (pcx340) and pBAD18-chaperone expressing Y. enterocolitica strains were inoculated into $3 \mathrm{ml}$ of $\mathrm{BHI}$ broth + tet \pm carb and grown at $26^{\circ} \mathrm{C}$ overnight. The following day, the cultures were diluted 1:20 in BHI + antibiotics and grown at $26 \mathrm{C}$ for $1 \mathrm{hr} .1 \mathrm{mM}$ IPTG and $13 \mathrm{mM} \mathrm{L}$-Arabinose were added to the cultures and were grown for 2 more hours at $26^{\circ} \mathrm{C}$. Monolayers were washed twice with HBSS, and infected with Y.enterocolitica at an MOI of 10 . After $1 \mathrm{hr}$ of infection at $37^{\circ} \mathrm{C}$, the monolayer was washed twice and fresh DMEM was added. The infection progressed for 2 more hours at $37^{\circ} \mathrm{C}$ in $5 \% \mathrm{CO}_{2}$. Infected cells were washed 3 times with $\mathrm{HBSS}$ and topped with 100ul of HBSS. The 1xCCF2/AM substrate was prepared as per the

manufacturer's manual (Invitrogen) and $20 \mathrm{ul}$ was added to each Ill. Infected cells were incubated at RT for $1.5 \mathrm{hrs}$ in the dark prior to fluorescence detection at 450 and $520 \mathrm{~nm}$ with a fluorimeter (excitation at $410 \mathrm{~nm}$ ).

\section{Enterropathogenic E.coli and Salmonella typhiumurium translocation assays.} Enterropathogenic E.coli or Salmonella typhimurium was inoculated into LB broth + tetracycline and chloramphenicol and allowed to grow at $37^{\circ} \mathrm{C}$ overnight. Cultures were diluted 1:33 in fresh LB broth + antibiotics and allowed to grow to mid-log phase at $37^{\circ}$ 
$\mathrm{C}$ (shaking for Salmonella, no shaking for EPEC). Bacteria were pelleted, washed and resuspended in PBS and added to $80 \%$ confluent HeLa cell monolayers at a multiplicity of infection (MOI) of 50. For the EPEC and SPI-1 assays, the monolayers were infected for 2 hours prior to the washing and CCF2/AM steps (see above), while the Salmonella were allowed to infect cells for 9 hours in the SPI-2 assay at an MOI of 10. 
CHAPTER III

\section{IDENTIFYING INTERACTIONS BETWEEN TARP AND TYPE III CHAPERONES FROM CHLAMYDLA TRACHOMATIS}

Since TARP translocation is a critical event that ensures the uptake and survival of Chlamydia, I sought to identify any type III secretion chaperones that may facilitate its translocation. From earlier in silico studies, I knew that three such type III class I (effector) chaperones were predicted in C. trachomatis genome. I manually aligned the predicted secondary structures of the open reading frames CT043, CT088, and CT663 with known class I effector chaperones from other pathogenic bacteria (Figure 2). In this figure I annotated the aforementioned chaperones as Slc1, Scc1, and Slc2. Previous

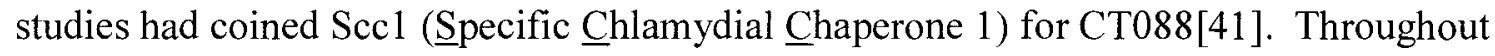
this study I refer to CT043 and CT663 as Slc1 and Slc2 for SycE-Like Chaperone 1 and 2 , respectively.

The predicted chlamydial chaperones shared the $\alpha-\beta-\beta-\beta-\alpha-\beta-\beta$ secondary structure characteristic of type III effector chaperones. Interestingly, all three putative chaperones were reported to be present in the elementary body proteome, [143-146] suggesting that these chaperones may function in the translocation of EB-associated effectors. These findings convinced us to test these putative chlamydial chaperones for their ability to interact with the invasion-associated effector TARP. 


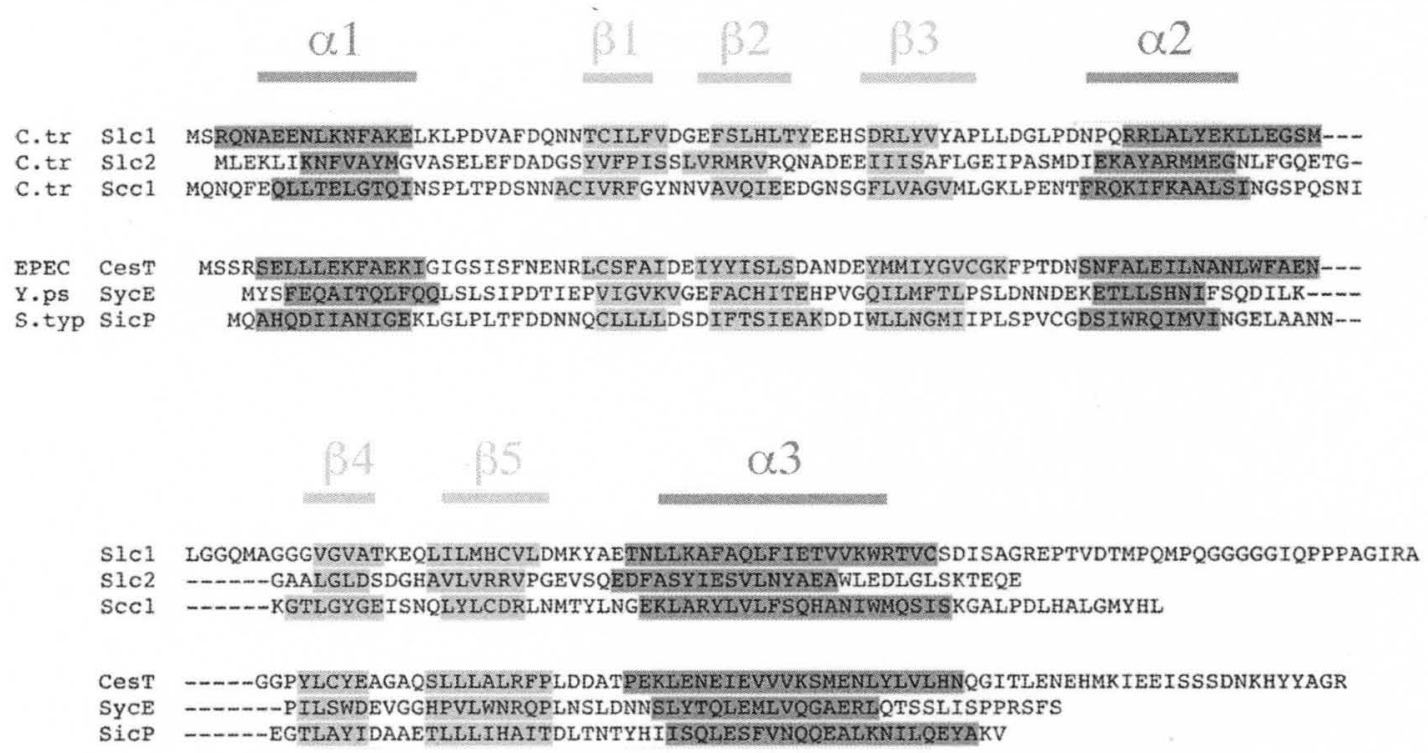

\section{Figure 2. Secondary structure alignment of type III chaperones.}

Three C.trachomatis proteins share predicted secondary structural features characteristic of known type III export chaperones. Primary amino acid sequence alignment of known type III export chaperones CesT (EPEC), SycE (Yersinia enterocolitica) and SicP (Salmonella enterica serovar typhimurium) with the putative export chaperones Slc1 (CT043), Slc2 (CT663) and Scc1 (CT088) of C.trachomatis. Secondary structure was predicted using Jpred and sequences aligned manually to according to the $\alpha-\beta-\beta-\beta-\alpha-\beta-$ $\beta$ - $\alpha$ signature characteristic of some type III export chaperones. Residues predicted to form $\alpha$-helices and $\beta$-strands are highlighted dark grey and light grey, respectively. 


\section{Interaction of the N-terminal 200 amino acids of TARP with the chaperone Slc1.}

Since chaperones typically bind their effectors within the N-terminal 30-150 amino acids, I chose to test the N-terminal 200 amino acids of TARP for binding by the three putative chlamydial chaperones Slc1, Slc2, and Scc1. I utilized two independent approaches - co-immunoprecipitation and the bacterial two-hybrid system to confirm a chaperone-binding partner for TARP. Both of these approaches alloId us to identify interactions between native recombinant proteins and have both been used previously to identify type III chaperone:effector interactions $[38,147]$. Co-immunoprecipitation is a directed blunt-force approach for identifying in vitro interactions, while the bacterial twohybrid assay allows for real-time interaction of expressed proteins and is conducive to quantitative analysis.

For bacterial expression in the co-immunoprecipitation assays, I cloned the Nterminal 200 amino acids of L2 TARP into pET200 (Figure 3) and expressed the recombinant $\mathrm{N}$-terminus of TARP with an N-terminal 6XHIS tag (HIS -TARP $^{1-200}$ ). Each of the predicted chlamydial chaperones were cloned into pET101 (Figure 5) and expressed to have C-terminal FLAG tags. A stop codon was included after the FLAG tag to exclude the vector-encoded 6XHIS tag from the chaperone open reading frame. Expression of recombinant TARP and chaperones such that they had different tags alloId us to purify interacting proteins using two different immobilization approaches: HIS $_{6^{-}}$ TARP $^{1-200}$ with Ni-Nta magnetic beads or chaperone-FLAG with M2 Anti-FLAG agarose. The separate recombinant tags for TARP and chaperones $\mathrm{w}$ ere also important for immunodetection by Western blot analysis. 
The open reading frames of the N-terminal 200 amino acids of TARP were amplified from C.trachomatis Serovar L2, Mouse pneumonitis, C.pneumoniae, and C.caviae by PCR. (Fig 3) Slc1, Scc1, and Slc2 were amplified from C.trachomatis serovar L2 genomic DNA by PCR. (Fig 5) The purified PCR products were cloned into Invitrogen's directional cloning vectors (pET200 and pET101) by using the energy stored in a bond between the 3' phosphate of the linear vector and Vaccinia virus topoisomerase I to join the 5' CACC overhang from PCR products with the GTGG overhang of the linear vector. This allows for directional blunt-end annealing of the PCR products to the linear vectors without any digestion and ligation steps. Transformation of these cloned vectors into chemically competent Top 10 cells gave a high yield on antibiotic-selective plates, and colonies were checked for correct inserts by colony PCR. (Figures 4 and 6) Plasmid DNA from each positive clone was confirmed for the correct inserts by sequencing (Genomic Core-Baxter II, University of Louisville). 

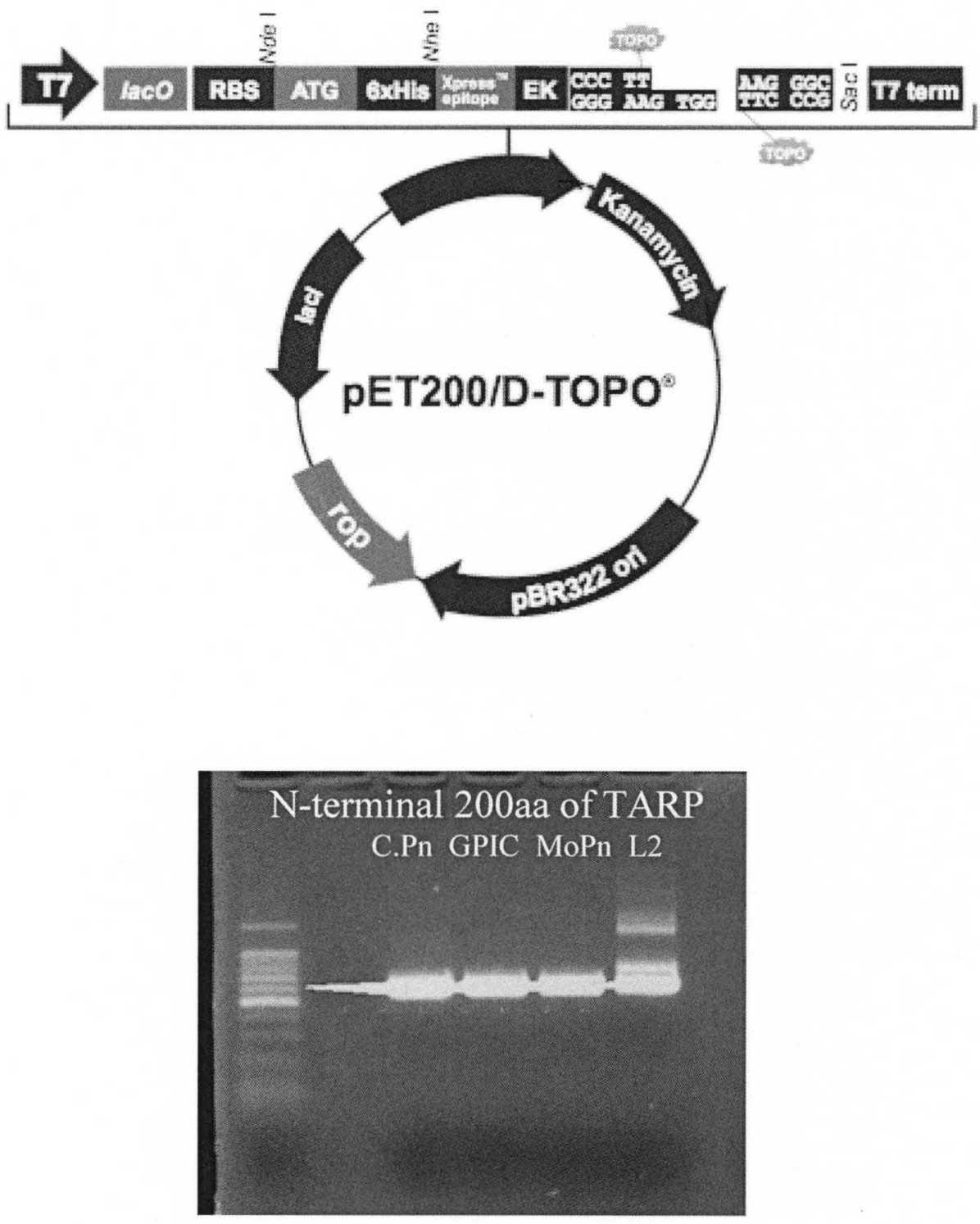

Figure 3. Amplification of TARP inserts for cloning into pET200.

(Top) Vector diagram of the pET200 directional topo cloning vector from Invitrogen. (Bottom) PCR-amplified inserts of the N-terminal 200 amino acids of TARP from different Chlamydial species are displayed on a 1\% agarose gel. 

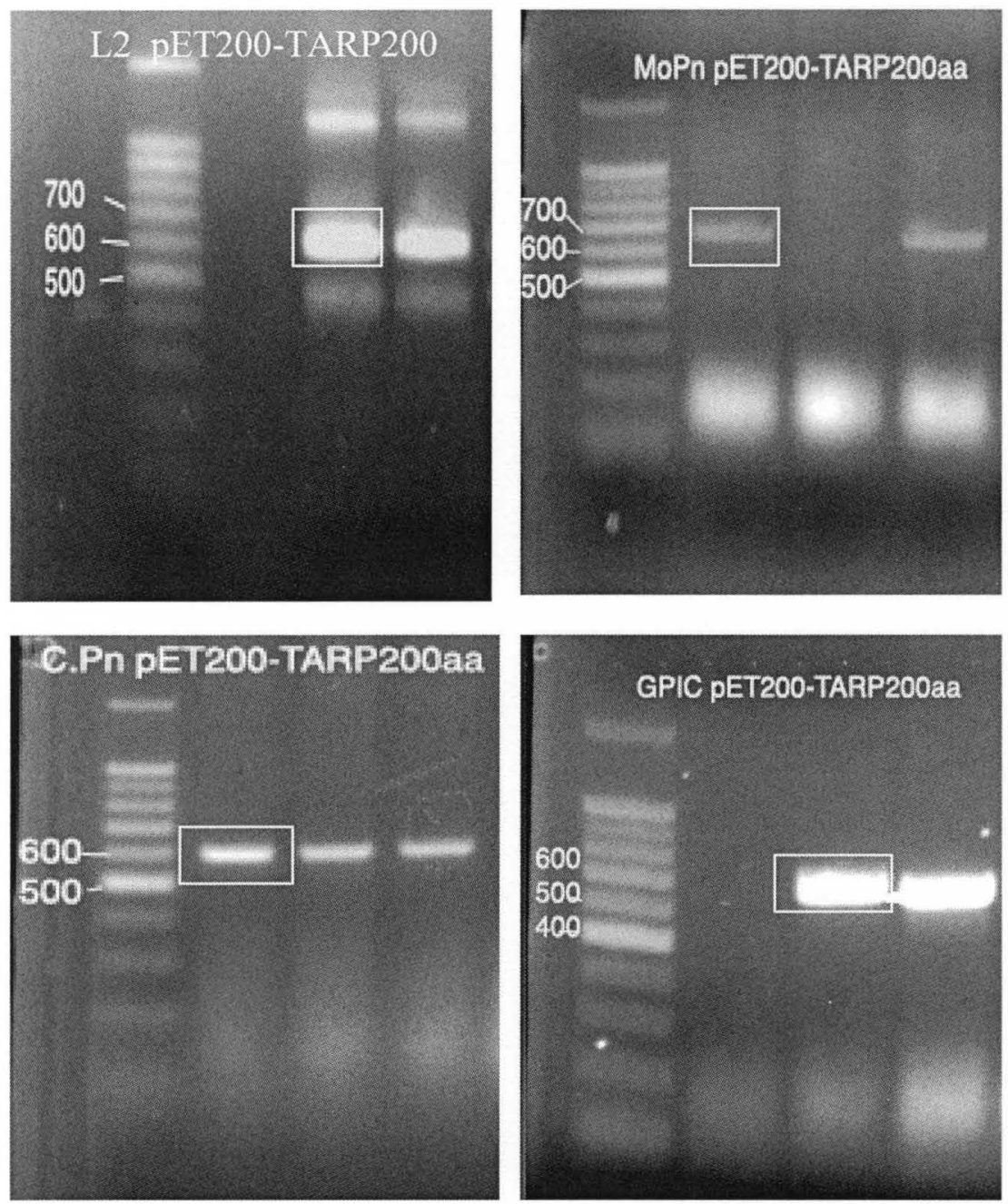

Fig 4. Colony PCR of transformants of pET200- TARP ${ }^{1-200}$.

Colony PCR was performed on kanamycin-selected transformants and the $1.2 \%$ agarose gels displayed here indicate the presence of the TARP200aa inserts for each Chlamydial species in the $\mathrm{pET} 200-\mathrm{TARP}^{1-200}$ vector. 

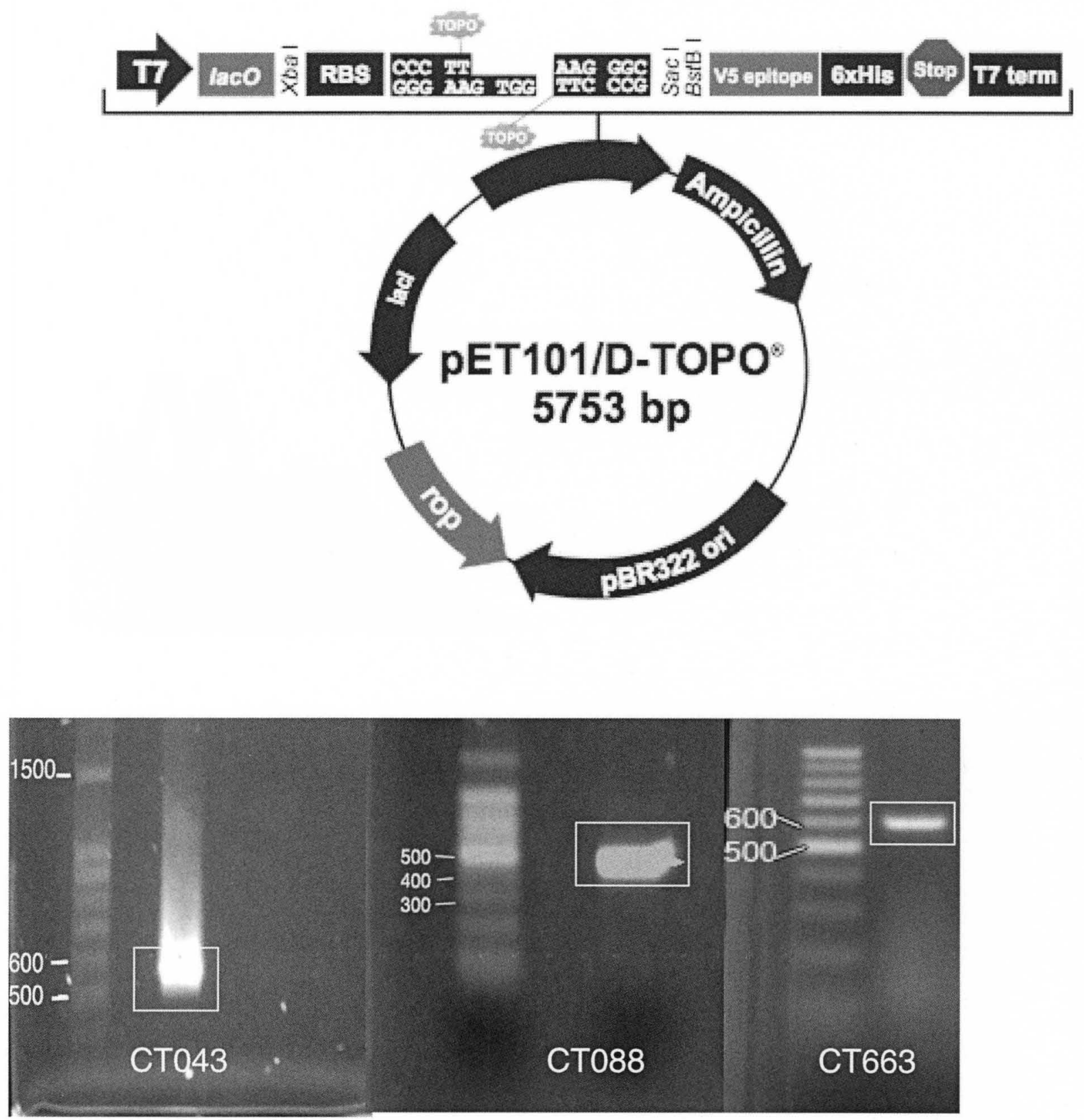

Figure 5. Amplification of chaperone inserts for cloning into pET101.

(Top) Vector diagram of the pET101 rectional topo cloning vector from Invitrogen. (Bottom) PCR-amplified inserts of the putative type III secretion chaperones from $C$. trachomatis serovar L2 are displayed on a 1\% agarose gel. 


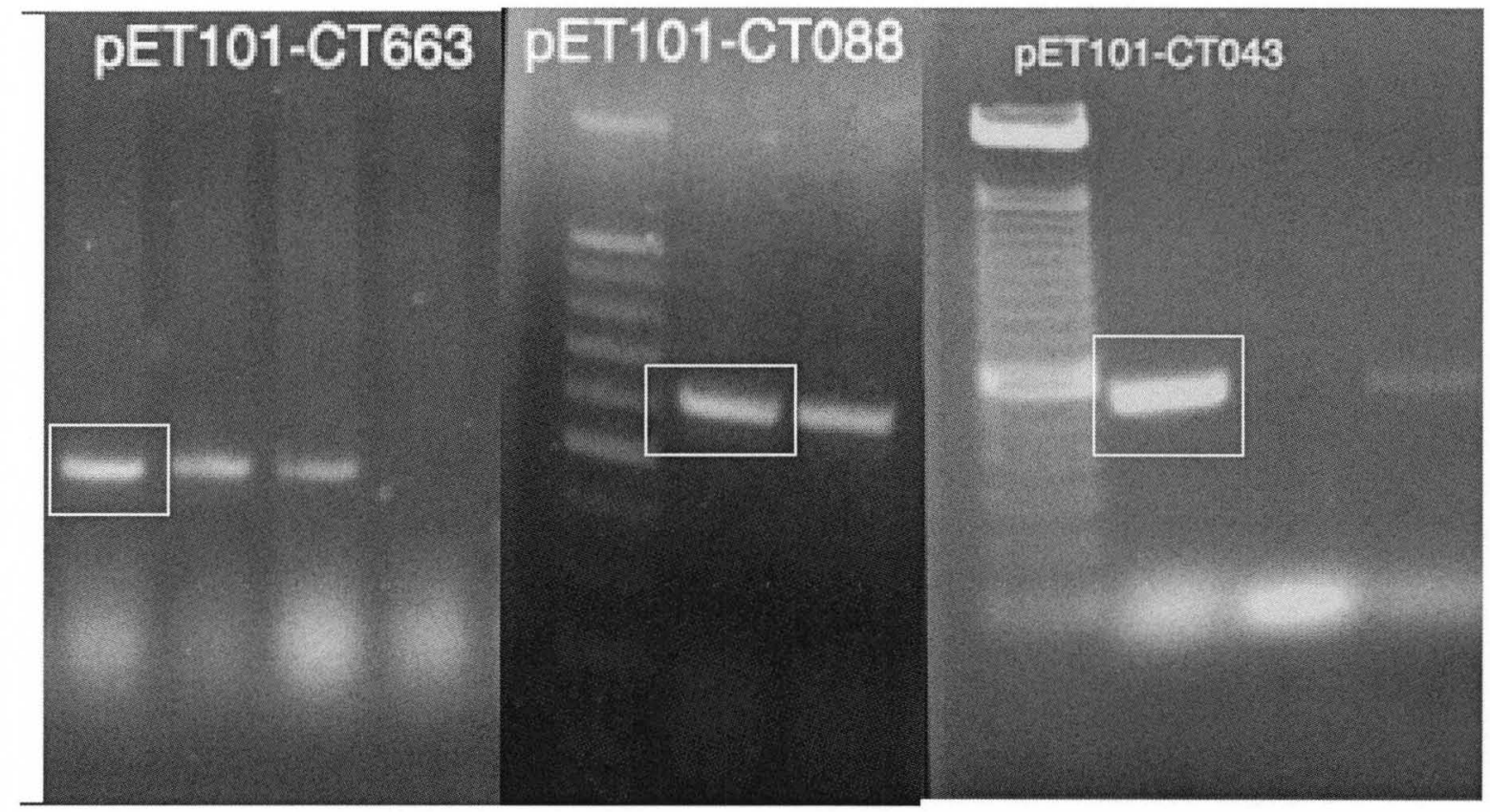

Fig 6. Colony PCR of transformants of pET101-chaperones.

Colony PCR was performed on kanamycin-selected transformants and the $1.2 \%$ agarose gels displayed here indicate the presence of C.trachomatis serovar L2 CT663, CT088, and CT043 inserts in the pET101 vector. 
Cleared BL21 star E.coli lysates containing HIS $_{6}-\mathrm{TARP}^{1-200}$, chaperone-FLAG, or a mixture of the 2 lysates were incubated with M2 Anti-FLAG agarose for 4 hours at $4^{\circ} \mathrm{C}$ to allow protein complexes to form and become immobilized on the agarose. After 5 wash steps to remove any non-specific proteins, I eluted the protein complexes with 250 $\mathrm{mM} 125 \mu \mathrm{g} / \mathrm{ml}$ 3XFLAG peptide. The specific retention of TARP by the chaperones was monitored in the flow-through, wash, and elution fractions by Western blot analysis (Figure 7).

The M2 Anti-FLAG beads retained $\mathrm{HIS}_{6}-\mathrm{TARP}^{\mathrm{I}-200}$ in the presence of recombinant Slc1, but not Slc2 or Scc1 indicating a specific interaction with Slc1, but not the other chaperones. HIS $_{6}-\mathrm{TARP}^{1-200}$ was present in all of the co-immunoprecipitation samples as evidenced by its presence in the flow-through fraction, therefore the lack of interaction with Slc2 and Scc1 was not due to an absence of TARP in the combined lysates. I can also eliminate excess protein forcing an interaction because the low level of Imidazole $(20 \mathrm{mM})$ in the wash buffer in combination with the multiple wash steps should eliminate any non-specific interactions. One minor caveat of this approach is that mixing of separately expressed, prefolded proteins may not be ideal for studying their native interaction. 


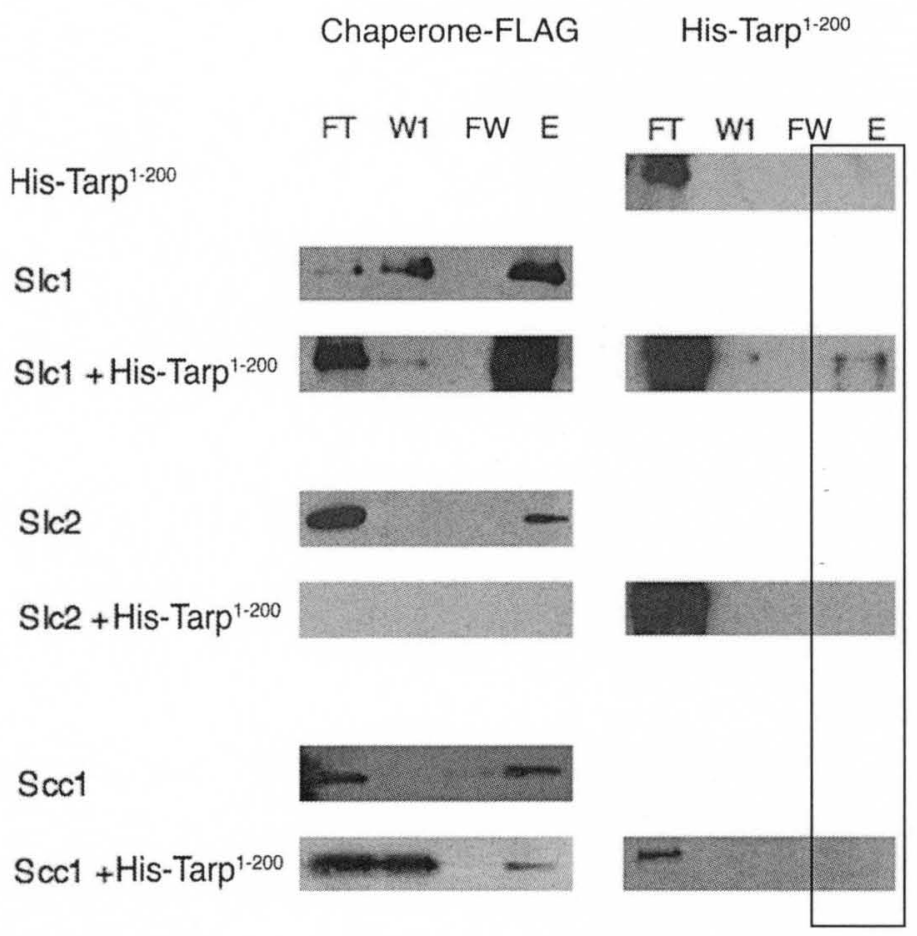

Figure 7. Pull-down of TARP by chlamydial chaperones from singly expressed lysates. Immunoblots of flow-through (FT), first wash (W1), final wash (FW), and elution (E) fractions following M2 Anti-FLAG co-immunoprecipitation of Slc1-FLAG, Slc2-FLAG or Scc1-FLAG in the presence or absence of His $_{6}$-TARP ${ }^{1-200}$ from laboratory E.coli lysates following individual expression were detected using anti-FLAG and anti$\mathrm{His}_{6}$ antibodies, respectively. 
To confirm the observed Slc1 binding to $\mathrm{HIS}_{6}-\mathrm{TARP}^{1-200}$, I performed the reverse co-immunoprecipitation reaction in which TARP was immobilized to Ni-Nta magnetic beads and monitored the different fractions for Slc1-FLAG content by Western blot (Figure 8). In this experiment, lysate from a BL21star E.coli strain that co-expresses Slc1-FLAG and $\mathrm{HIS}_{6}-\mathrm{TARP}^{1-200}$ was incubated with Ni-Nta magnetic beads. Fractions were processed and detected as stated previously. As expected, I found that the Ni-Nta magnetic beads when co-expressed with $\mathrm{HIS}_{6}-\mathrm{TARP}^{1-200}$ retained high levels of recombinant Slc1, but none was retained in its absence.

In addition, I tested whether co-expression of Scc1 or Slc2 with TARP enhanced their interaction (data not shown) and I was unable to detect any Scc1 or Slc2 in the elution fraction, giving confirmation that TARP interacts specifically with the putative chaperone Slc1. I also observed a relatively higher level of TARP retained in the reverse co-immunoprecipitation experiment that utilized lysates from E. coli co-expressing both TARP and Slc1. 


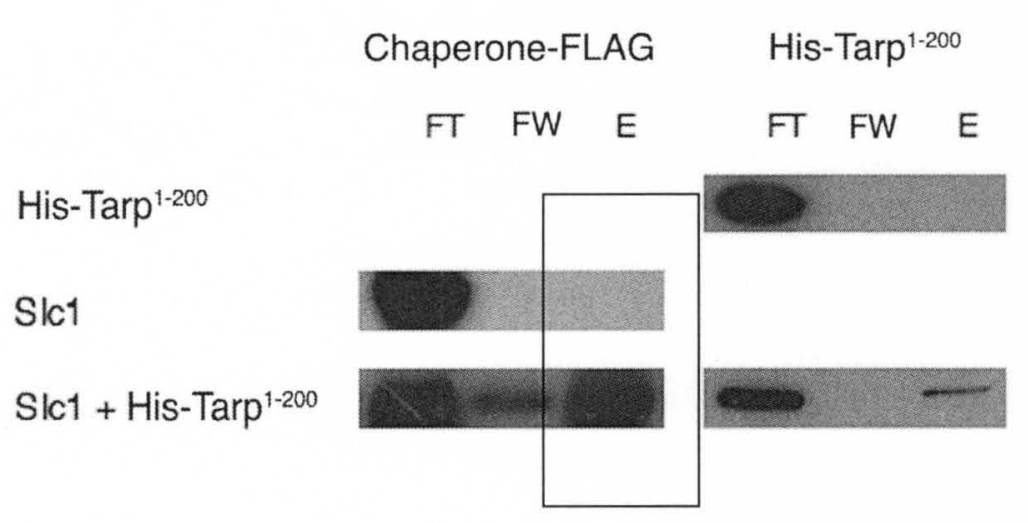

Figure 8. Pulldown of chaperones by TARP following coexpression

Immunoblots of flow-through (FT), final wash (FW), and elution (E) fractions following $\mathrm{Ni}-\mathrm{Nta}$ immunoprecipitation of $\mathrm{His}_{6}-\mathrm{TARP}^{1-200}$ from laboratory E.coli lysates following expression of TARP ${ }^{1-200}$-His $_{6}$ or Slc1-FLAG alone, or after co-expression as indicated. Slc1-FLAG and TARP ${ }^{1-200}{ }_{-H_{1}}$ is $_{6}$ were detected using anti-FLAG and anti-His 6 , respectively. 
To confirm independently the Slc1-TARP interaction demonstrated by coimmunoprecipitation, the bacterial two-hybrid technique was used[148, 149]. The Nterminus of chlamydial TARP was fused to the C-terminus of the Bordatella pertussis adenylate cyclase (cya) 18 -fragment (Cya 18 -TARP) in the vector pUT18C (Fig 9). Each of the chlamydial chaperones, including the recently described Mcsc, which has a chaperone-like activity, was cloned into the vector pKT25 (Figure 9) and expressed as Cterminal fusions to the 25 fragment of cya $\left(\mathrm{Cya}_{25}-\mathrm{Slc1}, \mathrm{Cya}_{25}-\mathrm{Sccl}, \mathrm{Cya}_{25}-\mathrm{Mcsc}\right.$, and $\mathrm{Cya}_{25}$-Slc2). The TARP and chaperone inserts were amplified by PCR and ligated into the $\mathrm{XbaI}$ and $\mathrm{Kpnl}$ sites of the pUT18C and pKT25 (Figures 10 and 11) vectors, respectively. The ligated vectors were transformed into BL21star and transformants were checked for the correct inserts by colony PCR followed by DNA sequencing (Fig 10 and 11). 

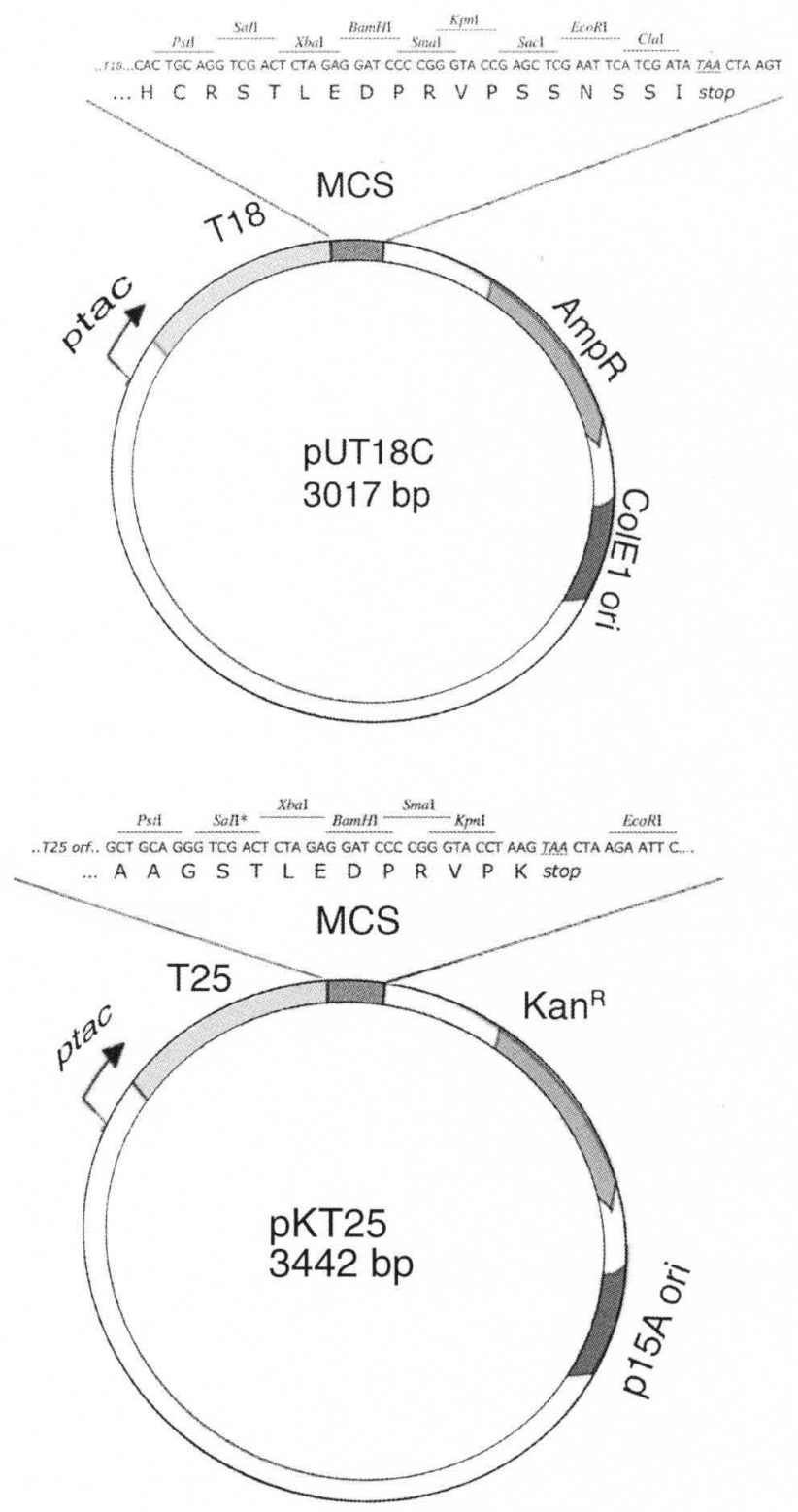

Figure 9. Diagram of the pUT18C and pKT25 $\beta$-lactamase fusion vectors. (Top) Diagram of the pUT18C vector. (Bottom) Diagram of the pKT25 vector. Colony PCRs of ampicillin-selected pUT18C-TARP00 transformants separated on a $1 \%$ Agarose gel. 

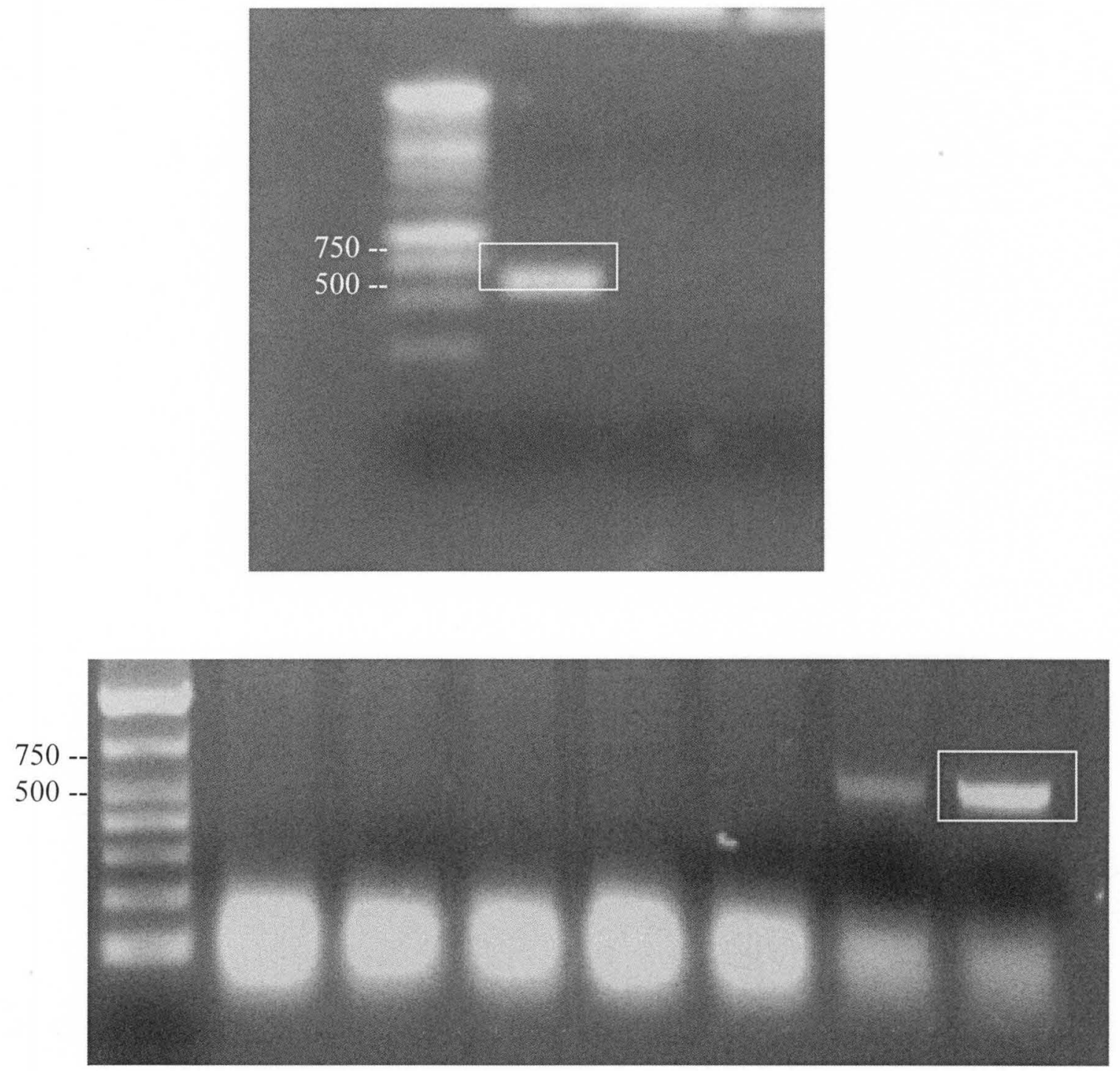

Figure 10. Cloning of the N-terminus of TARP into pUT18C.

(Top) PCR of the TARP200aa insert with XbaI and KpnI sites run out on a 1.2\% Agarose gel. (Bottom) Colony PCR of the ampicillin-selected pUT18C-TARP200aa transformants run out on a $1.2 \%$ Agarose gel. 

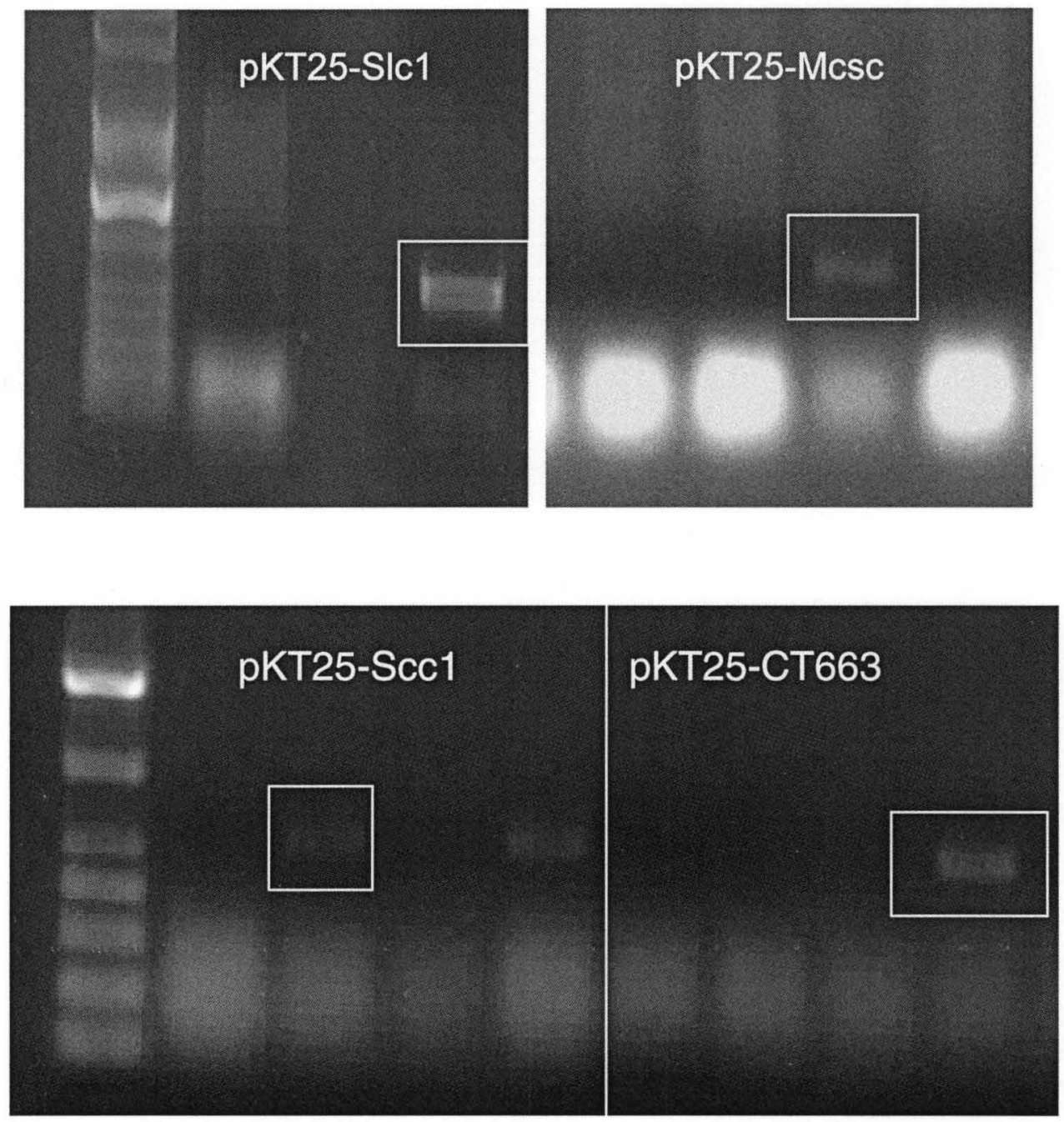

Figure 11. Confirmation of pKT25-chaperone transformants.

Kanamycin-selected pKT25 transformants were checked for their chlamydial chaperone inserts. Colony PCR for Slc1 (Top left), Mcsc (Top right), Scc1 (Bottom left) and CT663 (Bottom right) were separated on $1.2 \%$ agarose gels. 


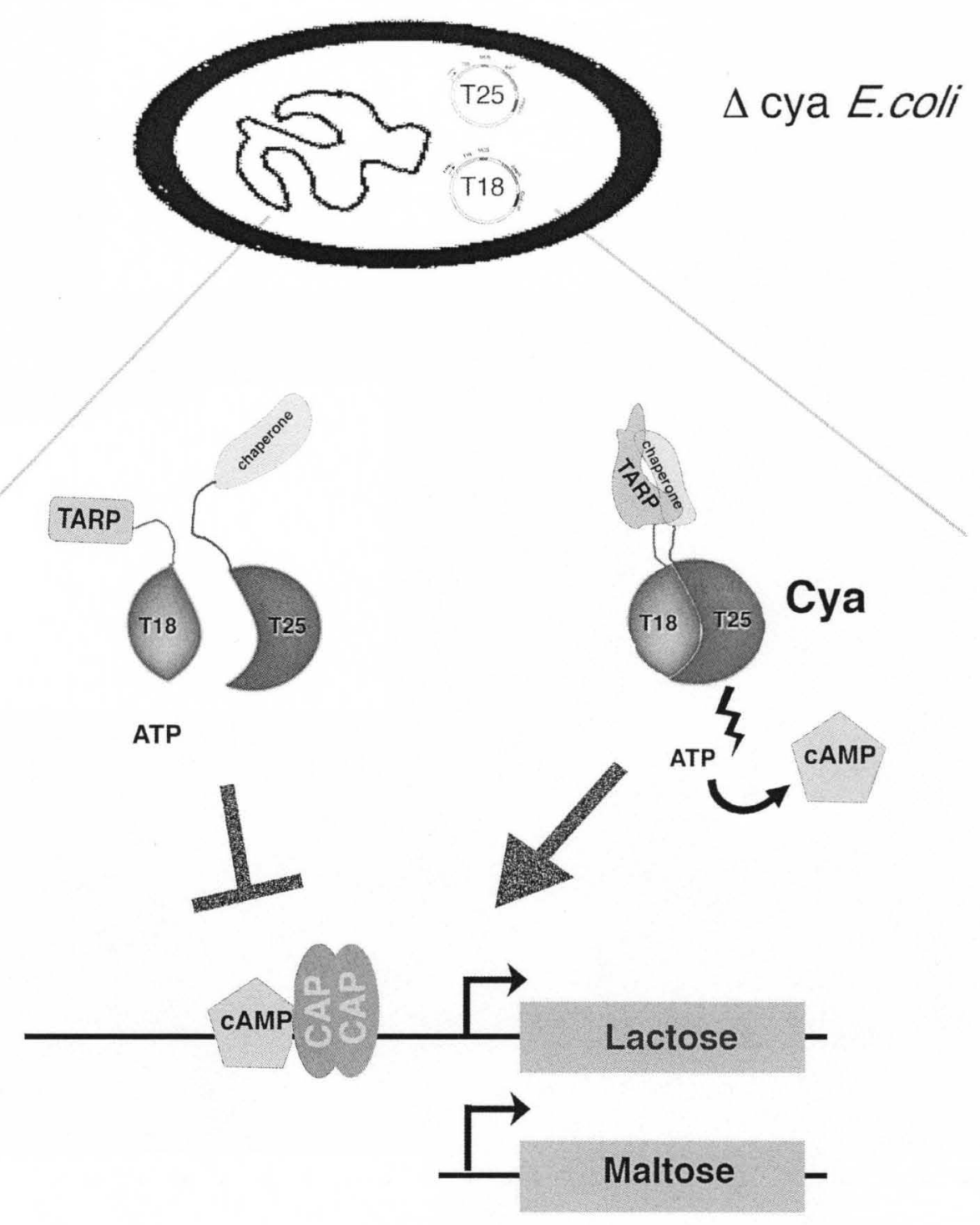

Figure 12. Diagram of the E.coli 2-hybrid system.

Co-expression of the T18 and T25 fusion proteins in adenylate cyclase-deficient (cya) E.coli yields two scenarios: 1. (Left) The fusion proteins do not interact and the cya toxin is not reconstituted. No cylic-AMP (cAMP) is formed and the lactose and maltose operons are not transcribed. 2. (Right) The fusion proteins interact, bringing together the T18 and T25 fragments and reconstituting the toxin. Cya mediates the formation of cAMP, and it further interacts with the catabolite activator protein (CAP) to co-activate transcription of lactose and maltose operons. 
These fusion vectors were then co-expressed in the cya deficient $E$. coli strain DHM1. In this bacterial 2-hybrid assay, interaction between fusion proteins results in the reconstitution of the adenylate cylcase (cya) enzymatic domain and catalyzes the conversion of ATP to (cAMP). Available cAMP, along with the catabolite activator protein (CAP) co-activates the maltose and lactose operons (Fig 12). Activation of the lactose operon leads to Beta-galactosidase ( $\beta$-gal) production, which cleaves the colorimetric substrate X-gal to yield blue colonies on $\mathrm{LB}+\mathrm{x}$-gal + IPTG plates. IPTG was included in the plates to relieve repression of the endogenous lactose operon by lacI. Activation of the maltose operon in colonies growing on McConkey $+1 \%$ maltose plates leads to a color change of the phenol red in the agar to fuchsia in the presence of acidic maltose degradation products[148, 149]. This bacterial 2-hybrid system gives quick, reproducible results on indicator plates, and protein-protein interactions can be quantified in a $\beta$-galactosidase reporter assay. I was able to detect an interaction between $\mathrm{Cya}_{18^{-}}$ TARP and $\mathrm{Cya}_{25}-\mathrm{Slcl}$ by blue and fuchsia colonies on X-gal and McConkey plates, respectively (Figure 13). Bacteria co-expressing $\mathrm{Cya}_{18}$-TARP and $\mathrm{Cya}_{25}$-Scc1, $\mathrm{Cya}_{25}-$ Mcsc, or $\mathrm{Cya}_{25}$-Slc2 were similar to the negative control, and did not produce an obvious color change to blue or fuchsia on indicator media. 


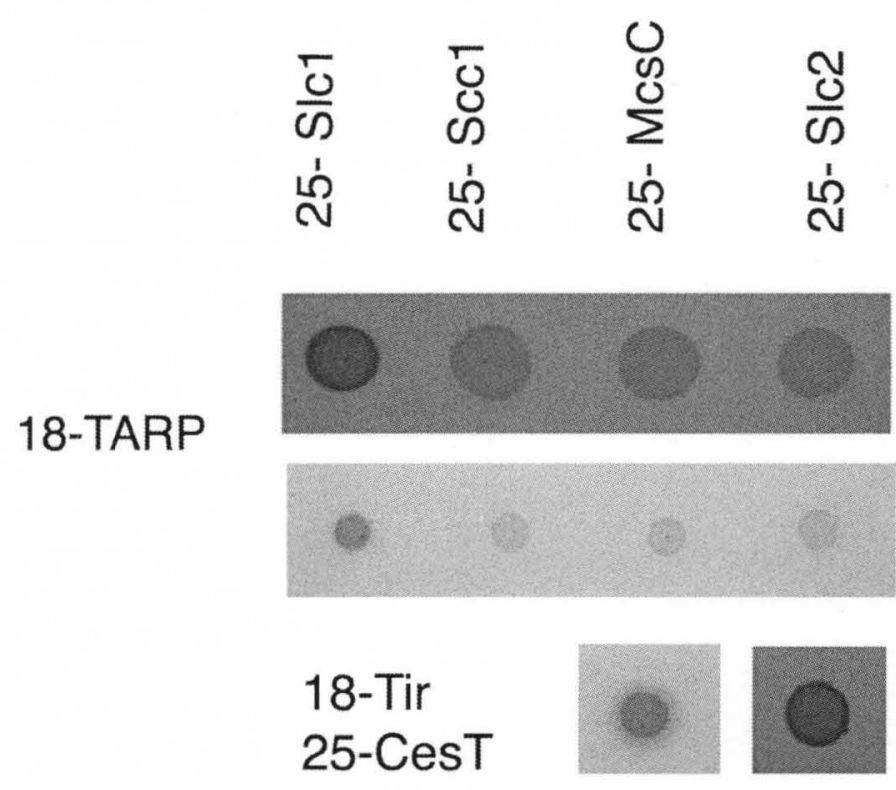

Figure 13. Bacterial 2-hybrid interactions between TARP and chaperones on indicator plates.

LB + X-gal + IPTG (left) or McConkey + maltose (right) agarose plates indicate interaction between co-expressed Cya15 or Cya18 fused chlamydial chaperones in the cya deficient E. coli strain DHM1. Upregulation of the lactose and maltose operons results from fusion-protein interaction and is indicated by blue or fuchsia bacteria. These results are representative of multiple trials. 
The results obtained from colonies on indicator plates were confirmed and quantified by monitoring $\beta$-galactosidase activity as previously described. Briefly, I permeabilized co-expressing DHM1 strains and measured the $\beta$-gal-mediated conversion of ONPG to the colorimetric molecule ONP. This measurement of $\beta$-gal activity was converted to Miller Units in order to take into account the cell number and length of time for color development in contrast to the indicator plate method that does not take bacterial cell numbers into account. The background level of interaction in the $\mathrm{Cya}_{18}{ }^{-}$ Empty/ Cya ${ }_{25}$-Empty co-expressing strain was subtracted from the Miller Units for each sample.

Extracts from DHM1 strains co-expressing Slc1 and TARP indicated 3-fold higher level of $\beta$-galactosidase production compared to the strain containing Slc2 and TARP (Figure 14). Both the Slc2/TARP and Scc1/TARP co-expressing DHM1 extracts contained roughly 5 -fold less $\beta$-galactosidase than the Slc1/TARP coexpressing strain. These results confirm the findings for a TARP-Slc1 interaction shown on indicator plates and in the co-immunoprecipitation assay. 


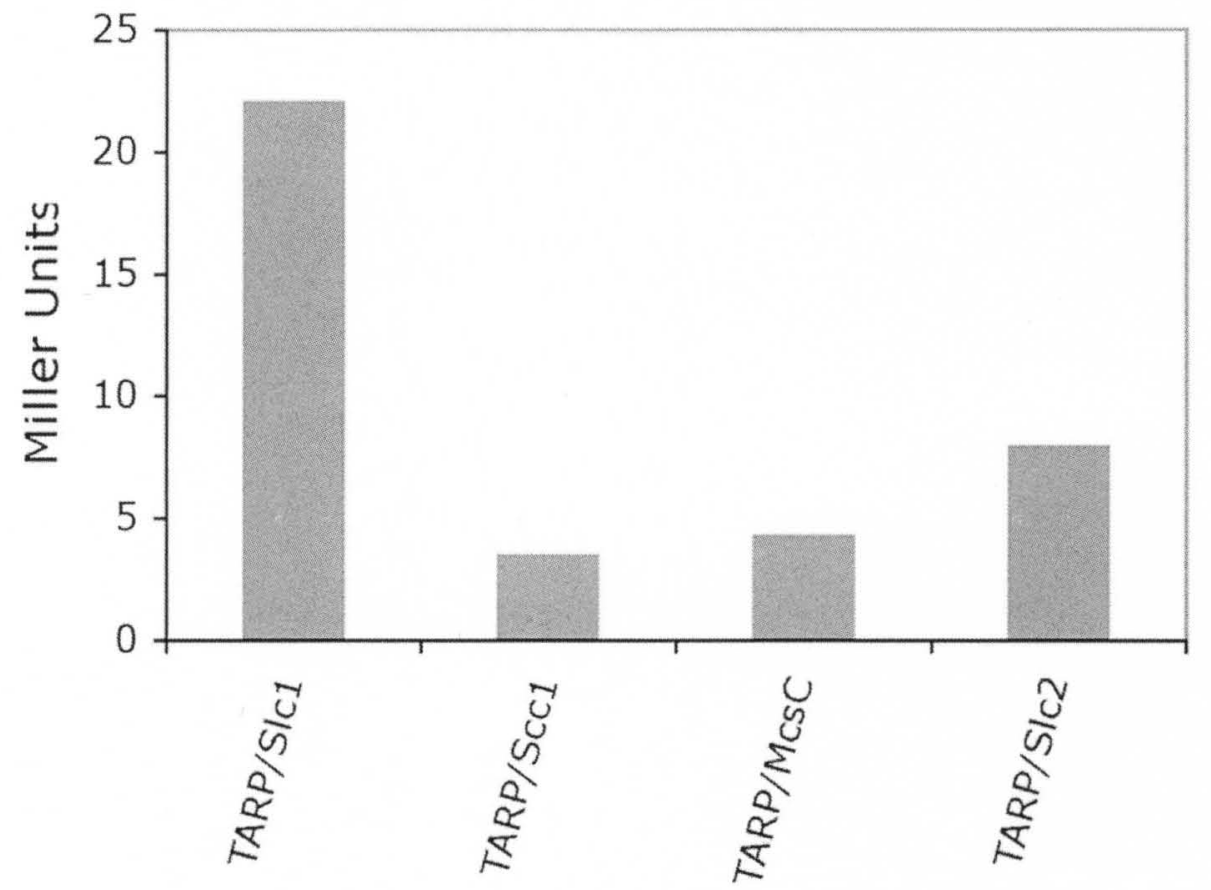

Figure 14. $\beta$-galactosidase activity of TARP:chaperone bacterial 2-hybrid interactions.

The relative beta-galactosidase activity of E.coli DHM1 strains co-expressing Cya ${ }^{18}$ $\mathrm{TARP}^{1-200}$ and $\mathrm{Cya}^{25}-\mathrm{Slc} 1, \mathrm{Cya}^{25}$-Slc2, $\mathrm{Cya}^{25}-\mathrm{Scc} 1$ or $\mathrm{Cya}^{25}$-Mcsc. Activity is expressed as Miller Units after subtraction of the background Cya ${ }^{25}$-Empty: Cya ${ }^{18}$-Empty interaction. Data is representative of multiple trials. 


\section{Interaction of SIcI with TARP from other Chlamydiae.}

Slc1 is highly conserved between chlamydial species, but there is a complete lack of amino acid similarity between the N-termini of different chlamydial TARPS.

Alignment of the secondary sequence of the N-terminal 200 amino acids of TARP from C.trachomatis L2, C.muridarum (MoPn), C.caviae (GPIC), and C. pneumoniae with the bioinformatics program ClustalW indicated conserved and semi-conserved features among the sequences (Figure 15). Several hydrophobic sites (in red) were spatially conserved between TARPs, and closer inspection reveals that these hydrophobic sites are flanked by conserved threonines and serines. However these features are conserved only through the $\mathrm{N}$-terminal 100 amino acids, indicating a possible chaperone binding site within these TARPs. This alignment led us to believe that Slc1 from the L2 serovar could possibly interact with the other chlamydial TARPS.

I first chose to look at the localization of putative chlamydial chaperones to full length Chlamydia caviae (GPIC) TARP due to its ability to form aggregates in mammalian cells. Full length TARP contains C-terminal actin-nucleating domains that are prone to aggregation and forms a characteristic string-of-pearls pattern when overexpressed in mammalian cells. I was able to acquire the GPIC-GFP-TARP vector encoding a C-terminal GFP (green fluorescent protein) from the Hackstadt lab at Rocky Mountain Laboratories. The three putative chlamydial chaperones Slc1, Scc1, and CT663 were PCR-amplified from L2 genomic DNA and subcloned into the Invitrogen $\mathrm{pENTR/D/TOPO}$ entry vector (Figure 16). The attL1 and attL2 recombination sites on this vector allow for recombination into a number of Destination vectors with the homologous sites attR and attB. For mammalian expression with an $\mathrm{N}$-terminally fused 
Mcherry protein I recombined the chaperones from the entry vector into pDEST-NMcherry (Figure 16). This allowed us to look for colocalization of the red chaperones with green aggregates of GPIC TARP.

The vectors GPIC-GFP-TARP and Mcherry-gus, Mcherry-Slc1, or Mcherry-Slc2 were co-transfected into Cos-7 cells and the co-localization of recombinant proteins was visualized by confocal microscopy (Figure 17). While the host cytosolic protein, gus localized some with TARP aggregates, this is probably just due to bleed-through from the green channel into the red channel. Slc2 localized to the TARP aggregates to the same extent as gus indicating a negative result for interaction. However, Slc1 overwhelmingly colocalizes with TARP aggregates in this assay. Of the two putative chaperones, only Slc1 was specifically recruited to TARP, confirming it as the specific type III chaperone for TARP and indicating that Slc1 can interact with the N-terminus of TARP from multiple chlamydial species. 


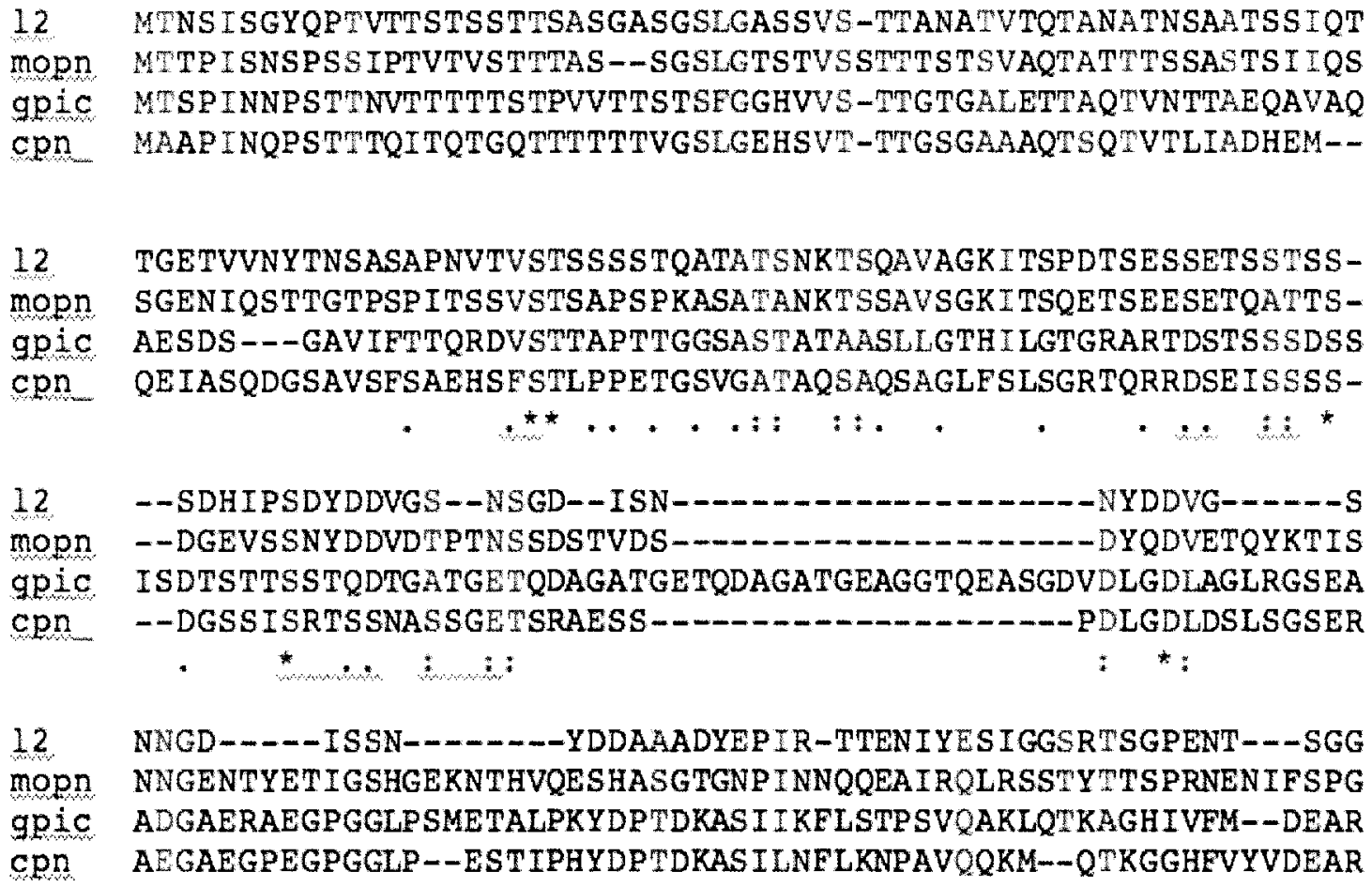

Figure 15. Amino acid alignment of the $\mathrm{N}$-terminus of TARP from different pathogenic Chlamydiae.

The N-terminal 200 amino acids of TARP from C. caviae (GPIC), C. pneumoniae (CPn), C. trachomatis (L2), and C. muridarum (MoPn) were aligned with clustalW. Residues in red indicate regions of conserved hydrophobicity, while light blue indicates conserved residues. 

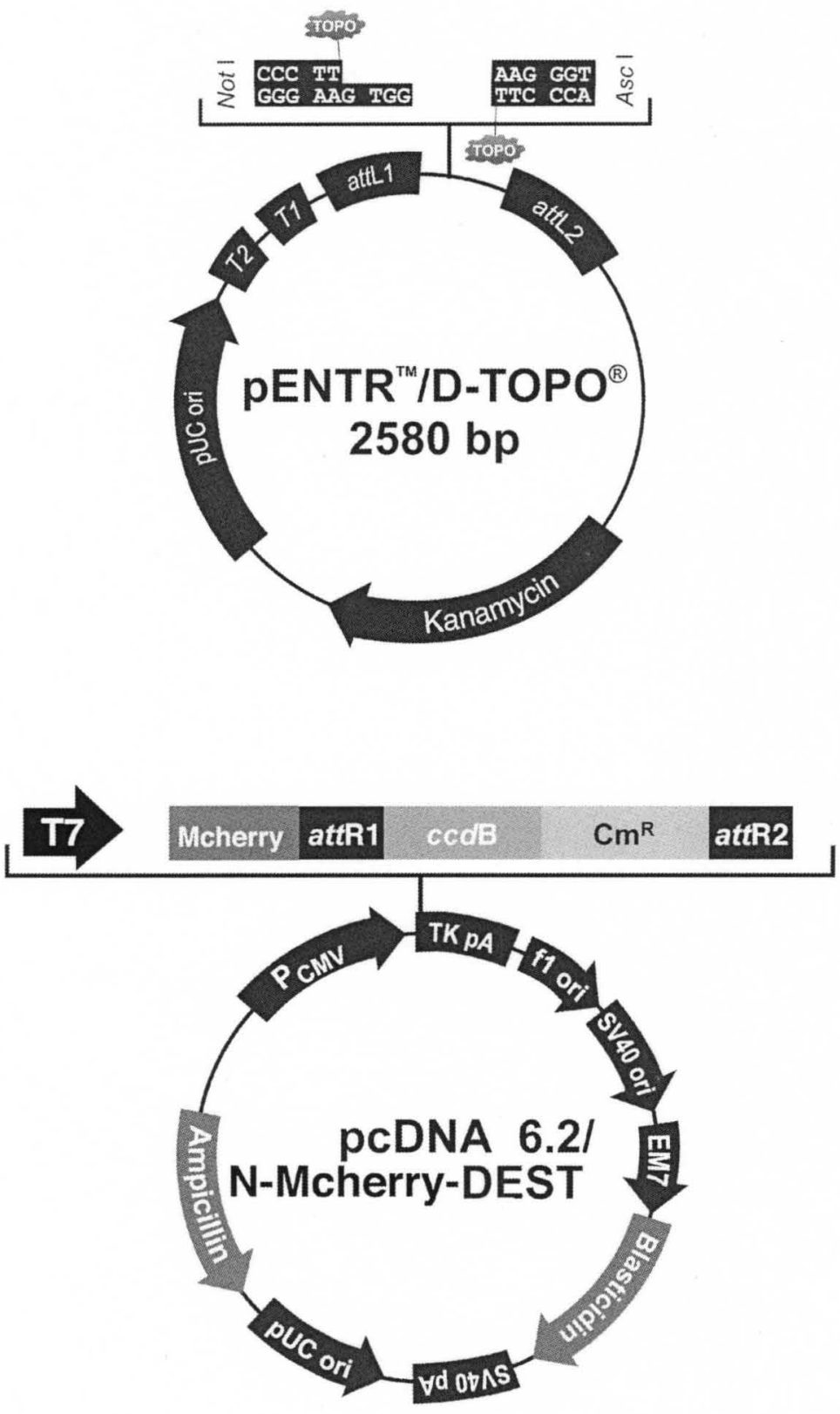

Figure 16. Diagram of the recombination vectors used to express chaperones in mammalian cells. 


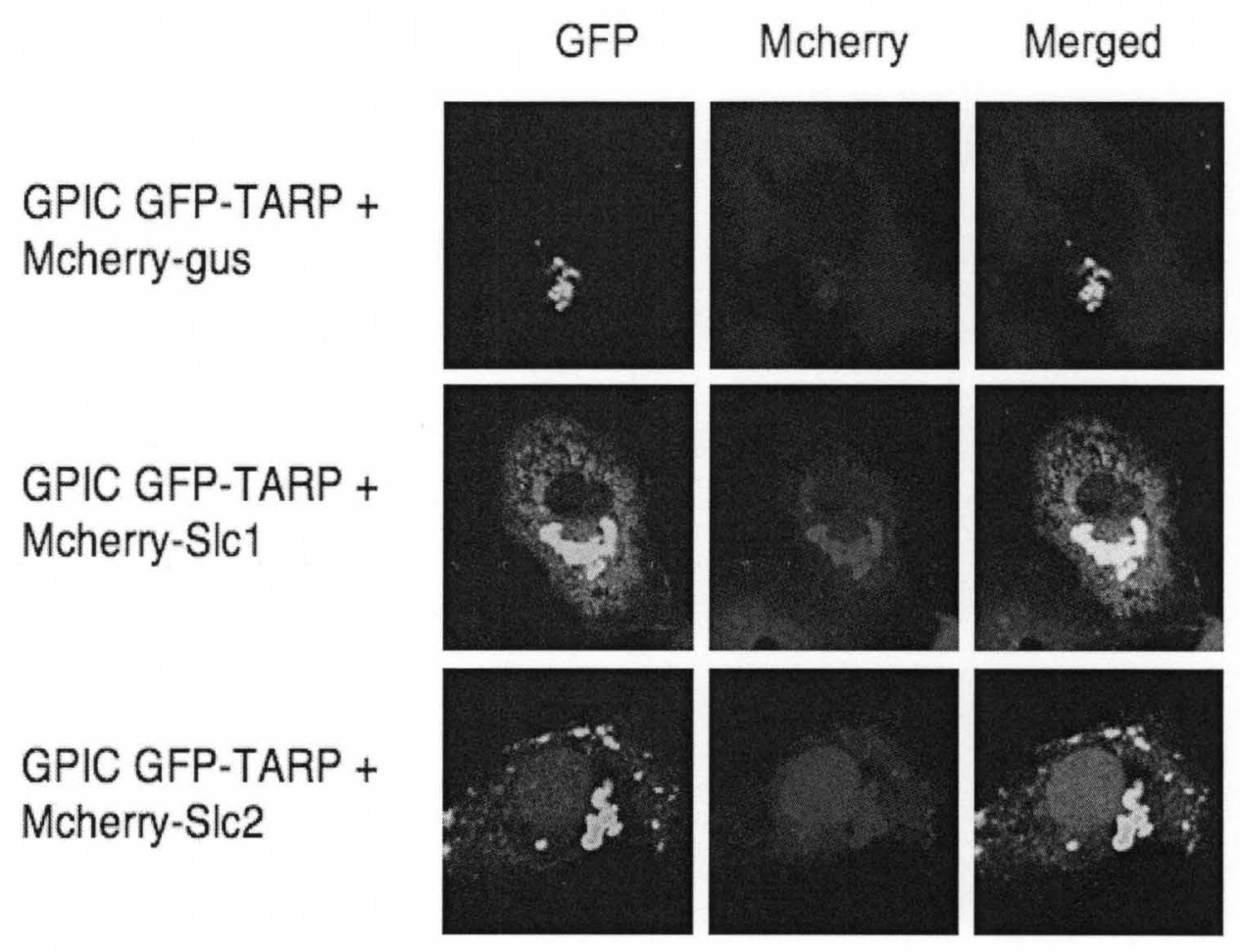

Figure 17. Localization of Slc1 to full-length $C$. caviae TARP aggregates

100X magnified images from confocal microscopy reveal colocalization of GPIC TARP and Slc1 in cotransfected Cos-7 cells. Cells were co-transfected with GPIC-GFP-TARP and Mcherry-gus (host cytosolic protein), Mcherry-Slc1, or Mcherry-Slc2. 
I continued to test the two other chlamydial TARPs for their ability to bind L2

Slc1. Interactions between C.pneumoniae and C.muridarum TARP with Slc1 were tested by co-immunoprecipitation with M2 Anti-FLAG agarose followed by immunoblotting. Addition of Slc1 and Cpn or Mopn TARP expression lysates allowed for their interaction which was confirmed by the presence of both of these TARP species in the Slc1containing elution fractions (Figure 18).

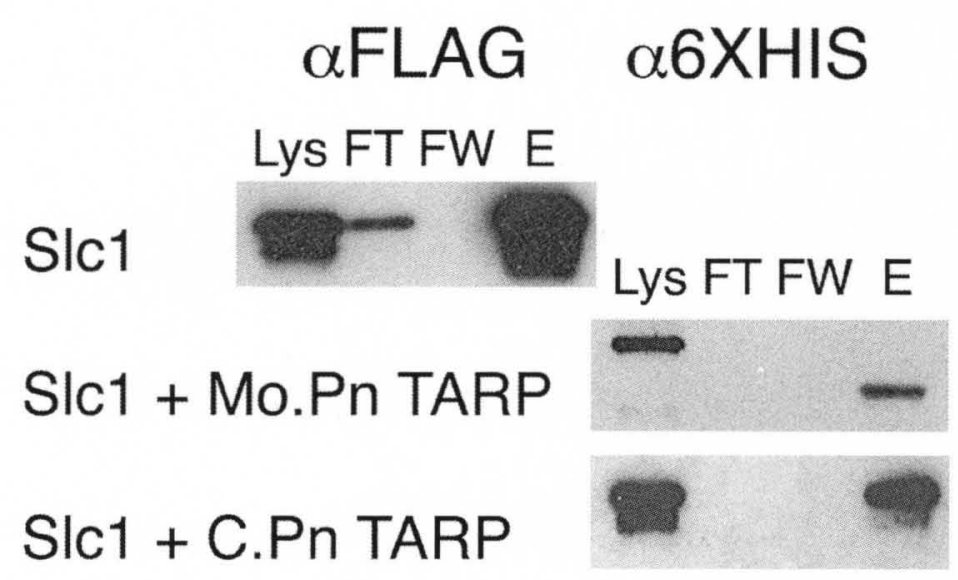

Figure 18. Pulldown of the N-terminus of $C$. muridarum and $C$. pneumoniae TARPs with Slc1.

Immunoblots of the lysates (Lys), flow-through (FT), final wash (FW), and elution (E) fractions from M2 Anti-FLAG immunoprecipitated recombinant Slc1 and the N-terminal 200 amino acids of C. muridarum (MoPn) or C. pneumoniae (C.Pn) TARPs are probed with their respective antibodies. 


\section{CHAPTER IV}

\section{IDENTIFICATION AND CHARACTERIZATION OF CHLAMYDIAL CHAPERONE: CHAPERONE INTERACTIONS}

Specific interaction between the chaperone Slc1 and TARP prompted us look deeper into the relationship of this chaperone: effector interaction. Translocation chaperones typically exist as either as a homodimer or a heterodimer prior to their interaction with their cognate effectors. Since our negative results for interaction between Slc2 or Scc1 and TARP only included possibly homodimers, I felt it necessary to see if the formation of Slc2-Slc1 or Scc1-Slc1 heterodimers could contribute to TARP

binding. I chose to characterize all possible chlamydial chaperone dimers to reveal any interactions that could be important for binding with TARP and other chlamydial effectors.

\section{Discovery of chlamydial chaperone: chaperone pairs}

Therefore, I investigated the ability of Slc1 to form homodimers with itself or heterodimers with either Slc2 or Scc1 using both co-immunoprecipitation and bacterial two-hybrid techniques. As before, I are taking advantage of separate recombinant tags on the proteins to purify protein complexes and to detect the protein composition of these complexes by Western blot analysis. I first wanted to detect whether Slcl can interact 
with itself or with other chaperones so I employed M2 Anti-FLAG agarose and Anti-Stag resin to purify chaperone dimers.

By co-immunoprecipitating Flag-tagged Slc1 (Slc1-F) with S-tagged Slc1 (Slc1S) on M2 Anti-FLAG beads, I was able co-elute them from the beads and thus demonstrate that SIc1 can multimerize with itself (Figure 19). However I could not detect any co-elution of Slc2-F with Slc1-S following immunoprecipitation on M2 agarose, indicating a lack of interaction between the two proteins. Similarly, I was also able to detect an interaction between Slc1-S and Slc1-F by co-immunoprecipitation with S-Rek resin, but not between Scc1-S and Slc1-F (Figure 20). In both coimmunoprecipitation assays detection of the Slc1: Slc1 interaction was minimal. This is not surprising considering the fact that many known type III secretion chaperones in other bacteria preferentially form dimers in solution, leaving few monomers around for potential dimer formation in our assay. 


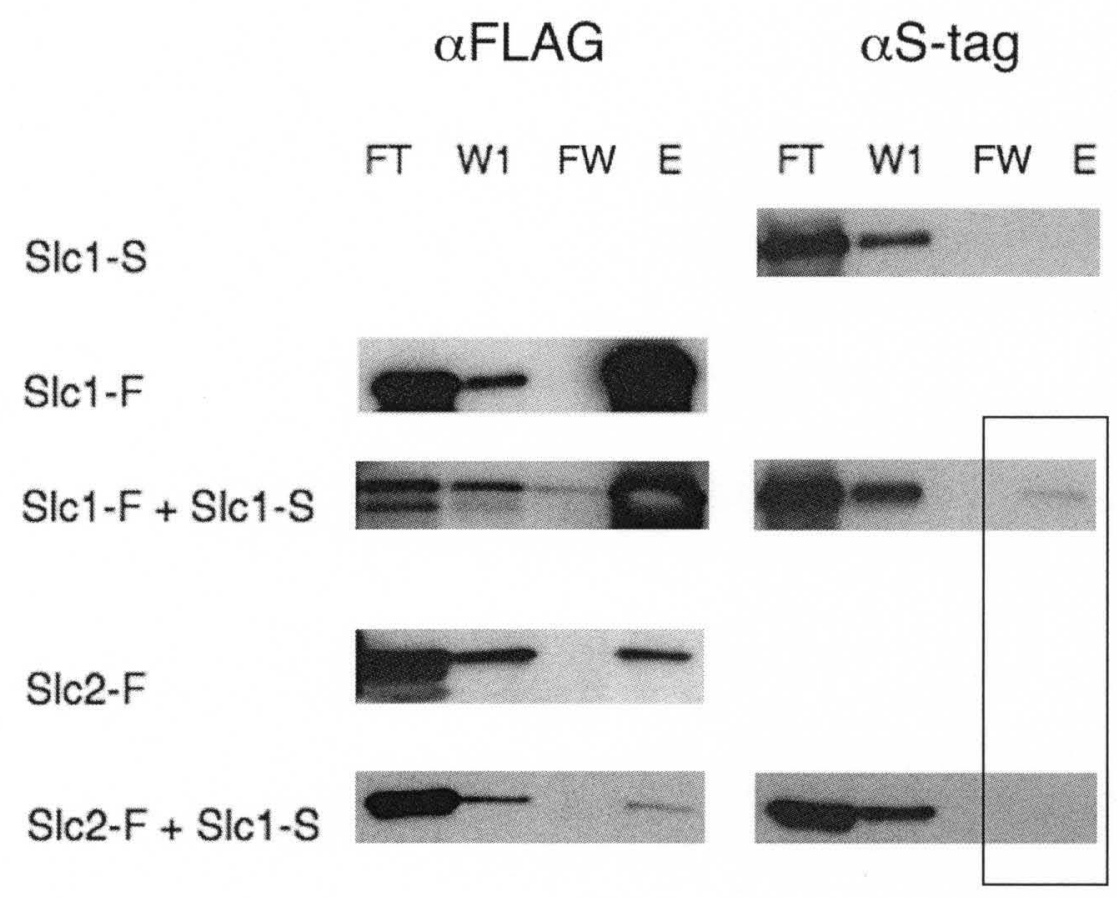

Figure 19. Pulldown of FLAG-tagged Slc1 and Slc2 in the presence of S-tagged Slc1. Immunoblots of the FT (flow-through), W1 (first wash), FW (final wash), and E (elution) fractions following M2 Anti-FLAG co-immunoprecipitation of FLAG-tagged chaperones (Slc1-F or Slc2-F) in the presence or absence of S-tagged Slc1 (Slc1-S) from coexpressed BL21 E. coli lysates were detected with $\alpha$ FLAG or $\alpha$ S-tag antibodies, respectively. 


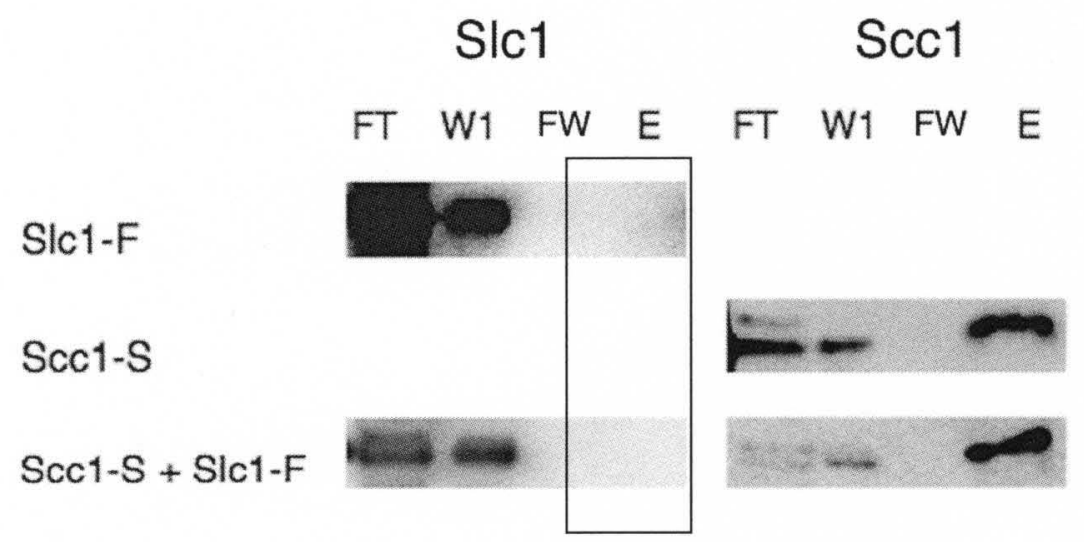

Figure 20. Pulldown of Slc1 in the presence of Scc1. Immunoblots of FT, FW, and E fractions following S-Rek resin immunoprecipitation of Scc1-S in the presence or absence of Slc1-F were detected with antibodies against their respective tags. 
Therefore I continued to investigate these interactions by E.coli 2-hybrid analysis, testing all possible chaperones: chaperone interactions. I was able to detect interactions between $\mathrm{Cya}_{18}$-Slc1:Cya ${ }_{25}-\mathrm{Slc1}$, Cya ${ }_{18}$-Mcsc: Cya25-Mcsc, and Cya ${ }_{18}-\mathrm{Slc} 2: \mathrm{Cya}_{25}$-Scc1 by blue and fuchsia colonies on X-gal and McConkey plates, respectively (Figure 21). The Mcsc:Mcsc and Slc2:Scc1 interactions had been previously identified in a recent yeast-two hybrid study [49]. I tested the expression of the $\mathrm{Cya}_{18}$-Scc1 and $\mathrm{Cya}_{25}-\mathrm{Slc} 2$ recombinant proteins to try to understand why the reciprocal 2-hybrid didn't indicate interaction, and was able to detect expression of both by coomassie staining (data not shown). All other chlamydial chaperone: chaperone interactions were similar to the negative control, and did not produce an obvious color change to blue or fuchsia on indicator media. 


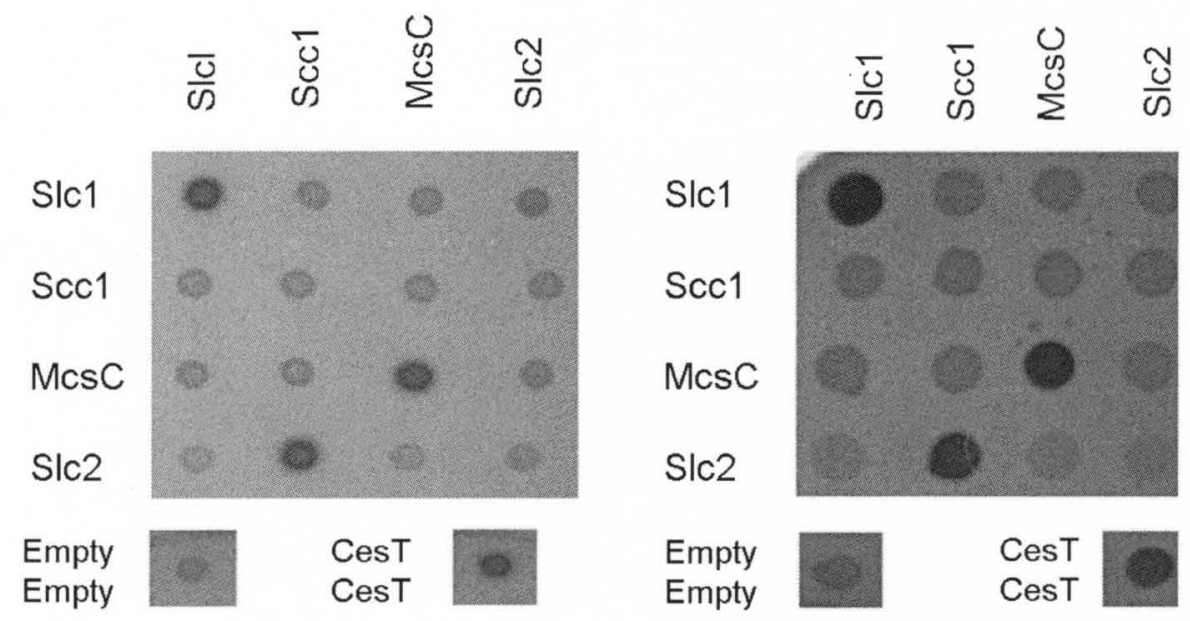

Figure 21. Bacterial 2-hybrid interactions between chlamydial chaperones. $\mathrm{LB}+\mathrm{X}$-gal + IPTG (left) or McConkey + maltose (right) agarose plates indicate interaction between co-expressed Cya15 or Cya18 fused chlamydial chaperones in the cya deficient E. coli strain DHM1. Upregulation of the lactose and maltose operons results from fusion-protein interaction and is indicated by blue or fuchsia bacteria. 
Quantitative analysis of the $\beta$-galactosidase activity of the $\mathrm{Cya}_{18}$-chaperone / $\mathrm{Cya}_{25}$-chaperone co-expressing strains indicated a very strong interaction between $\mathrm{Cya}_{18}$ Mcsc and Cya 18 -Mcsc, whereas the Slc1:Slc1 and Slc2:Scc1 interactions were stronger than the background chaperone: chaperone interactions (Figure 22). Other chaperone: chaperone interactions were higher than background levels but still lower than the interactions that had been previously confirmed on indicator plates. Taken together, these bacterial 2-hybrid data indicate a novel Slc1-Slc1 interaction and confirms the previous Mcsc-Mcsc and Slc2:Scc1 interactions that were discovered in a recent yeast 2-hybrid study. All together the co-immunoprecipitation and bacterial 2-hybrid results indicate that Slc1 forms homodimers, but not heterodimers. Given our previous findings that TARP does not interact with Scc1 or Slc2, it is likely that only Slc1 homodimers interact with TARP. 


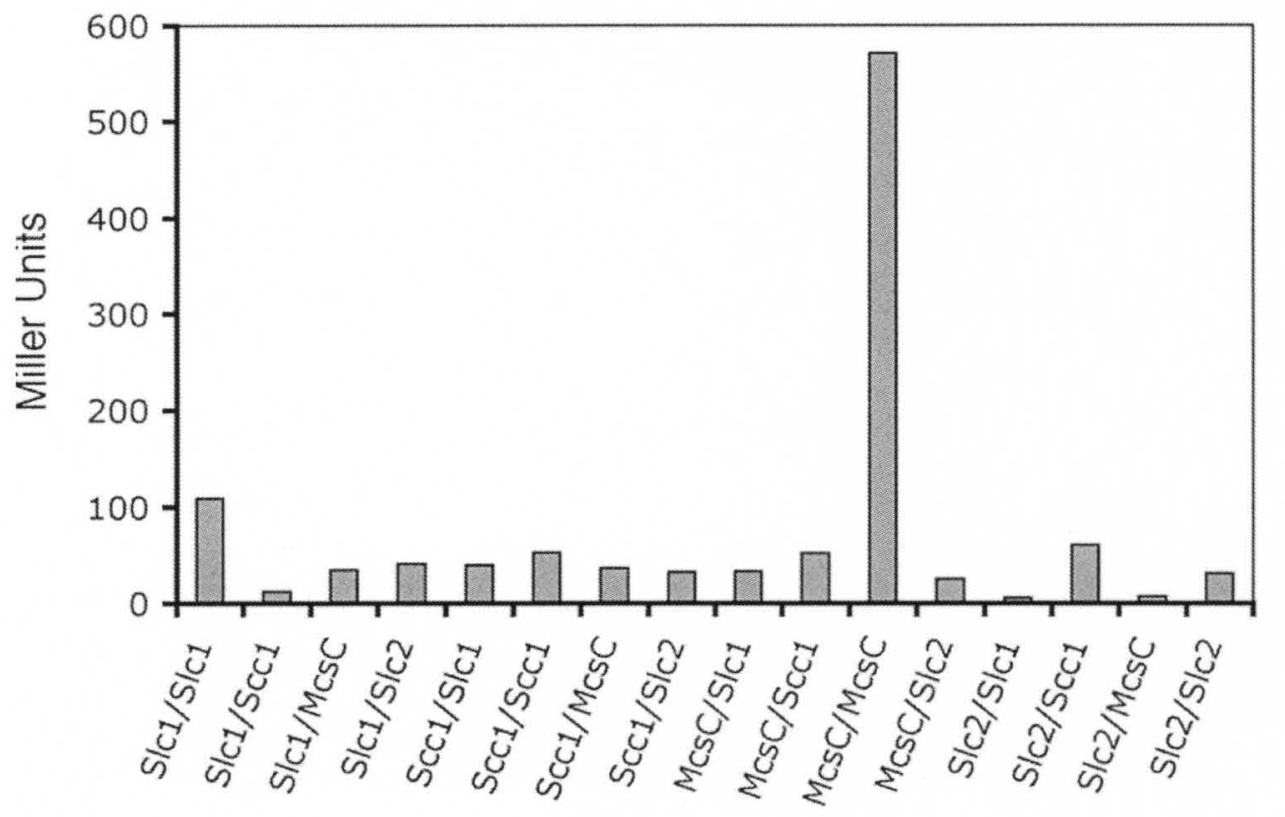

Figure 22. Quantitative analysis of bacterial 2-hybrid chaperone: chaperone interactions,

The relative beta-galactosidase activity of E.coli DHM1 strains co-expressing the recombinant $\mathrm{Cya}^{18}$-chaperones and $\mathrm{Cya}^{25}$-chaperones. Activity is expressed as Miller Units after subtraction of the background $\mathrm{Cya}^{25}$-Empty: Cya ${ }^{18}$-Empty interaction. Data is representative of multiple trials. 


\section{Determination of the molecular composition of Scl homo-complexes}

I expect the apparent Slc1:Slc1 interactions shown by co-immunoprecipitation and bacterial 2-hybrid analysis was a result of the formation of homodimers, however the rare case of trimer and tetramer forms of type III chaperones have also been observed to interact with type III effectors in other bacteria. I chose to detect all native Slcl complex forms by stabilizing their interaction with a crosslinker and separating the complexes on an SDS-PAGE gel. Since I wanted to crosslink M2-Anti-FLAG agarose purified Slc1 in PBS, I chose to use a hydrolysable crosslinker that could be reduced in the presence of $\beta$ mercaptoethanol (BME), but not in its absence. In addition I wanted to use a crosslinker that would react with the primary amines (mostly N-termini) of purified proteins. The compound DTSSP binds cysteines and primary amines at both ends and has been modified from its original non-hydrolyzable form, DSP, to be able to dissolve and react in solution. (Figure 23) Crosslinking of purified FLAG-Slc1 with the hydrolysable DTSSP reagent revealed the ability of this protein to form a dimer in the absence of the crosslinker; this dimeric complex was able to withstand denaturation in the SDS-PAGE gel (Figure 23). In fact, purified Slcl that was reduced (BME) and heated to $90^{\circ} \mathrm{C}$, but not crosslinked still showed faint dimerization at the expected $\sim 40 \mathrm{kDa}$ size. The addition a small concentration of crosslinker $(0.25 \mathrm{mM})$ revealed a higher molecular weight Slcl complex with a size $(\sim 60 \mathrm{kDa})$ consistent with trimeric species. Since noncrosslinked, non-reduced purified Slcl readily migrated to a dimer-sized position on a non-reducing SDS-PAGE gel it is clear that Slc1 forms stable dimers. 
<smiles></smiles>

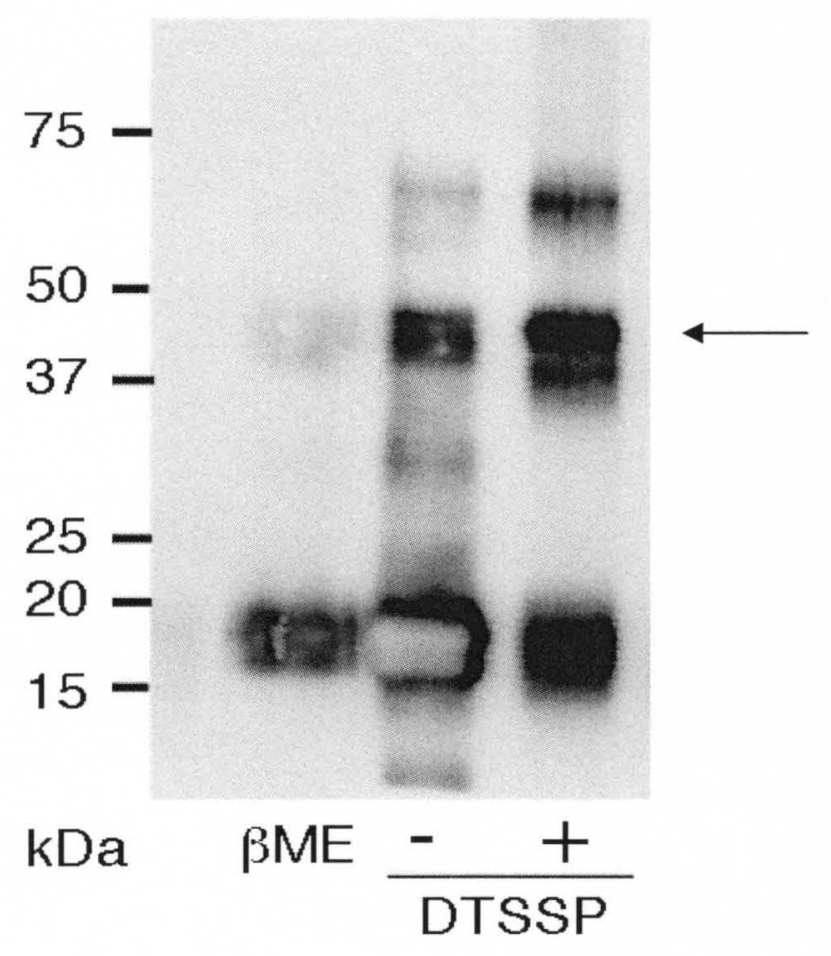

Figure 23. Stabilizing Slc1 complexes by the crosslinker DTSSP.

Multimerization of Slc1. (Top) Chemical structure of the crosslinker 3, 3'-Dithiobis (sulfosuccinimidylpropionate (DTSSP). (Bottom) An immunoblot of $\beta$-mercaptoethanol $(\beta \mathrm{ME})$, mock-treated $(-)$, or $0.25 \mathrm{mM}$ DTSSP crosslinked $(+)$ purified Slc1 was probed with $\alpha$ FLAG antibody following separation in 12\% SDS-PAGE gel under non-reducing conditions. 
While I was able to detect dimerization of Slc1 by crosslinking, the presence of a trimeric species required independent confirmation using gel filtration chromatography, which is a more gentle analytical technique that did not requiere crosslinking. Upon purification of Slc1-FLAG with M2 Anti-FLAG agarose, I loaded $50 \mu \mathrm{l}$ of $100 \mathrm{ng} / \mathrm{ml}$ purified Slc1 into a Superdex 200 10/300 GL column in PBS. The column washes the protein through sepharose gel with additional PBS. Larger species get stuck in the gel sooner, and thus get eluted from the column first. Prior to elution of $500 \mu \mathrm{l}$ fractions along the length of the gel, the relative abundance of the protein is detected at a wavelength of $280 \mathrm{~nm}$.

The relative abundance of protein in each of the eluted fractions is shown on the $y$-axis, while the relative size of each species (peak) is indicated by the elution fractions on the $\mathrm{x}$-axis. Each elution fraction was monitored for Slc1 content by Western blot. Higher molecular weight complexes are eluted earlier. Only one prominent peak was detected for the Slc1 complex and when compared to known molecular weight standards in PBS, it was determined to be $\sim 40 \mathrm{kDa}$ in size (Figure 24). Slc1 was detected in the TCA-precipitated elution fractions under the dimeric peak by immunoblotting. Crosslinking and gel filtration of purified Slcl indicates that it preferentially forms homodimers, indicating that this is the form it likely takes when interacting with TARP. 


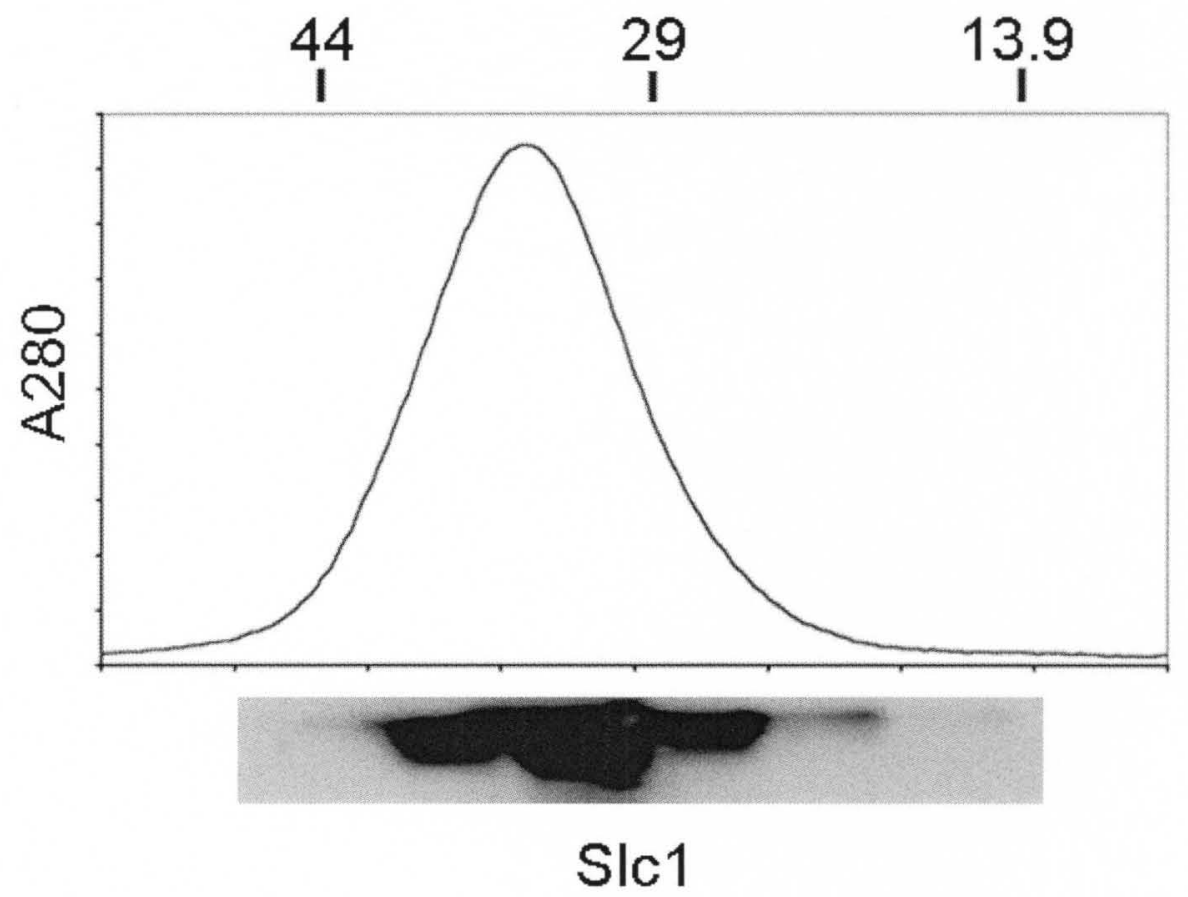

Figure 24. Detection of native Slc1 species by gel filtration chromatography

The relative abundance of native M2-purified Slc1 complexes were detected and eluted in $500 \mathrm{ul}$ fractions by gel filtration chromatography. An immunoblot of trichloracetic acid (TCA)-precipitated fractions was detected with $\alpha$ FLAG antibody. 
CHAPTER V

\section{FUNCTIONAL DETERMINATION OF SLC1 IN THE TRANSLOCATION OF TARP BY HETEROLOGOUS TYPE III SECRETION SYSTEMS}

\section{TARP: Slc1 complexes indicate the expected 2:1 stoichiometry of type III chaperone:}

\section{effector interactions}

I was able to detect a complex formed between Slc1 and TARP by detection of a shift in complex size between purified recombinant TARP and TARP co-expressed in the presence of Slc1on a silver-stained native gel and on its corresponding 6XHIS-probed immunoblot. Since native gels separate proteins based on their shape and size, it is difficult to extrapolate an approximate molecular weight of the complex (Figure 25).

To gain some insight into the composition of the complex formed by TARP and Slc1, a similar chromatography approach was used to analyze purified $\mathrm{HIS}_{6}$-TARP ${ }^{1-200}$ and the $\mathrm{HIS}_{6}-\mathrm{TARP}^{1-200} / \mathrm{Slc1-FLAG}$ complex. The chromatography conditions produced readily identifiable peaks for $\mathrm{HIS}_{6}-\mathrm{TARP}^{1-200}$ from Slc1-FLAG quite easily (Figure 26).

To determine the approximate size of the protein complex formed by TARP and $\mathrm{Slc1}$, the Ni-Nta agarose eluates from lysates containing co-expressed $\mathrm{HIS}_{6}$-TARP ${ }^{1-200}$ and Slcl-FLAG were subjected to gel filtration chromatography. Ni-Nta purified protein 
from the lysate containing $\mathrm{HIS}_{6}-\mathrm{TARP}^{1-200}$ was used as a reference control. The Slc1 and TARP content of the co-expressed elution fractions was detected with antibodies against their respective tags by Western blot. Since the co-expressed complex was purified with Ni-Nta agarose, I expect the sample to contain both monomeric TARP and TARP in a complex with Slc1. Here I see that TARP-containing elution fractions correspond to a peak between $13.9 \mathrm{kDa}$ and $29 \mathrm{kDa}$ and a peak $>75 \mathrm{kDa}$, while Slcl is present only in the elution fractions corresponding to the $>75 \mathrm{kDa}$ peak. These sizes are in agreement with monomeric TARP and a 1:2 complex of TARP to Slc1. Taken together with our Slc1 dimerization data, the results indicate that a dimer of the Slc1 chaperone forms a complex with one molecule of $\mathrm{HIS}_{6}-\mathrm{TARP}^{1-200}$ when co-expressed. In order to detect the Slc1TARP complex by chromatography, co-expression of Slc1 and TARP appeared to be necessary because the addition of pre-purified components failed to produce a similar $>75$ kDa-peak (Figure 27). 
Silver Stain

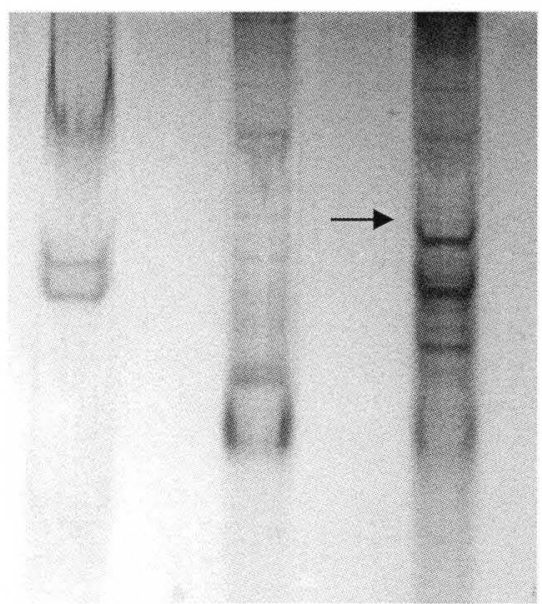

TARP Slc1 CO $\alpha 6$ XHIS

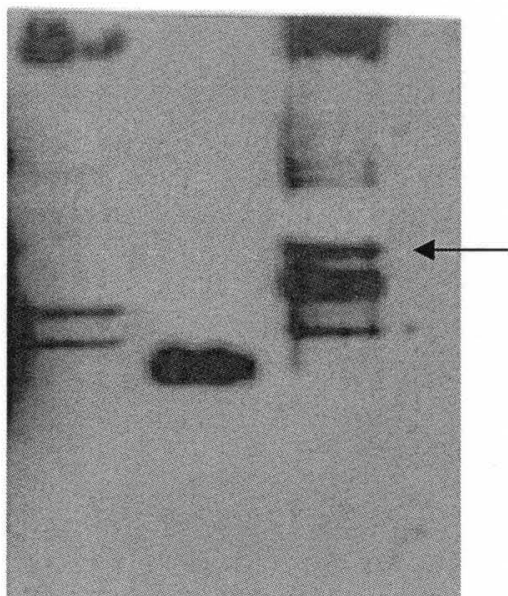

TARP SIc1 CO

Figure 25. Native gel shift of TARP: Slc1 complex

Native purified protein complexes are shown on both silver stain and Western blot. All protein species from the purified fractions are displayed on the silver-stained Native PAGE gel on the left. TARP-containing complexes are detected in the identical purified fractions with $\alpha 6$ XHIS antibody on the immunoblot on the right. Arrows indicate the complex formed under co-expressing conditions. 


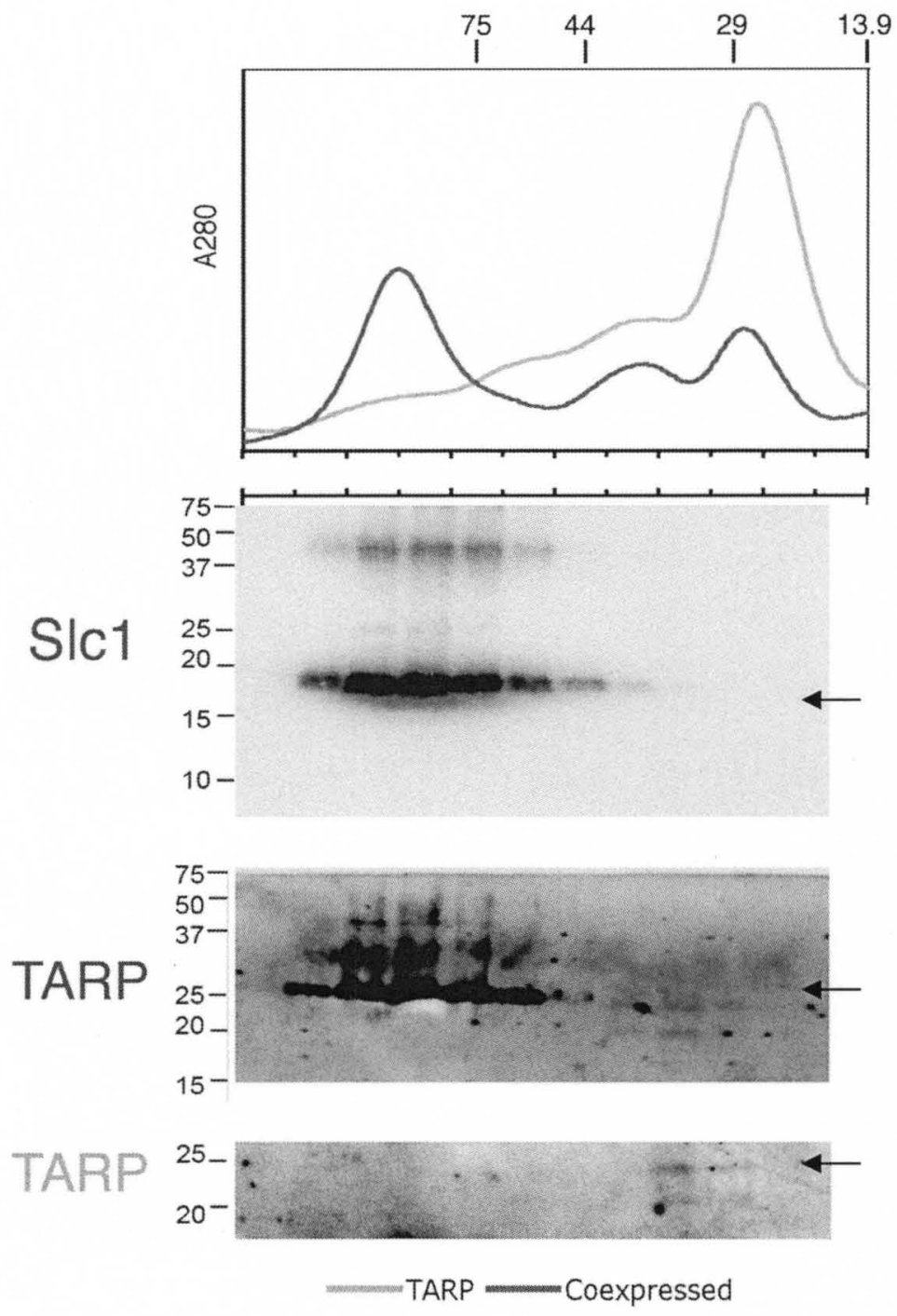

Figure 26. Detection of TARP: Slc1 native complex (coexpressed).

Separation and detection of co-expressed Slc1: TARP complexes. The relative abundances of native NiNta-purified TARP ${ }^{1-200}$ complexes alone or co-expressed with Slc1 were detected and eluted in $500 \mathrm{ul}$ fractions (top) by gel filtration chromatography. Immunoblots of the TCA-precipitated elution fractions were detected with $\alpha 6 \mathrm{XHIS}$ and $\alpha$ FLAG antibodies (bottom). 

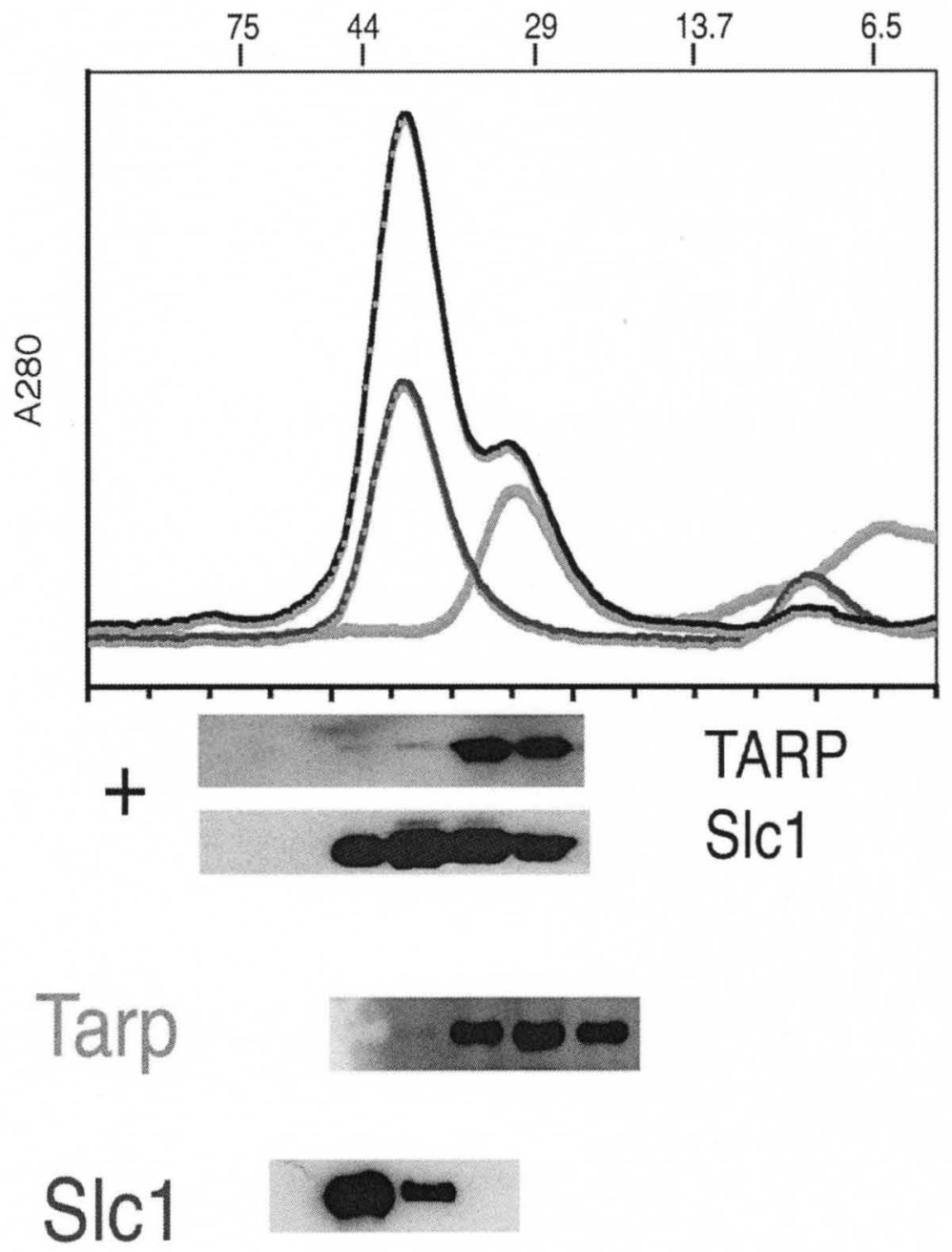

\section{Figure 27. Detection of TARP: Slc1 native complex (Combined).}

The separation and detection of M2-purified Slc1 (Red), Slc1 +TARP (Blue), or Ni-Ntapurified TARP (Green) was achieved by gel filtration chromatography. The peaks on the chromatogram correspond to the molecular weight standards (top). The relative abundances at A280 are indicated by the y-axis and the corresponding immunoblots of the $500 \mu \mathrm{l}$ fractions on the x-axis were TCA-precipitated and detected with $\alpha 6$ XHIS and $\alpha F L A G$ antibodies. 


\section{Translocation of TARP by heterologous type III secretion systems}

While the biochemical data suggests that Slcl interacts with TARP in a fashion typical of type III chaperone: effector complexes, I desired to know what role Slc1 plays in TARP translocation by the chlamydial type III secretion apparatus. Unfortunately, Chlamydia's lack of a genetically tractable system prevented us from directly testing Slc1's role in TARP's translocation from the chlamydial EB into mammalian epithelial cells. Fortunately, I was able to express our recombinant chlamydial proteins in the pathogenic bacteria Yersinia enterocolitica, Enteropathogenic E. coli (EPEC), and Salmonella typhimurium and detect TARP's translocation through their heterologous type III secretion apparatuses into Hela cells.

To accomplish this, I took advantage of an existing translocation assay that involved expressing possible effectors in the $\mathrm{pCX} 340$ vector that $\mathrm{C}$-terminally fuses them to enzymatically active $\beta$-lactamase, and the chaperones were cloned into an arabinoseinducible expression vector pBAD18-Cm (Figure 28). The chaperones were amplified from C. trachomatis L2 genomic DNA by PCR (Figure 29) and cloned into the KpnI and XbaI sites on the vector. A KpnI site was added downstream of the TARP ${ }^{1-200}$ open reading frame in the $\mathrm{pET} 200-\mathrm{TARP}^{1-200}$ vector by SLIM PCR (Figure 30), while TARP was cloned into the NdeI and KpnI sites of pCX340 (Figure 31). 

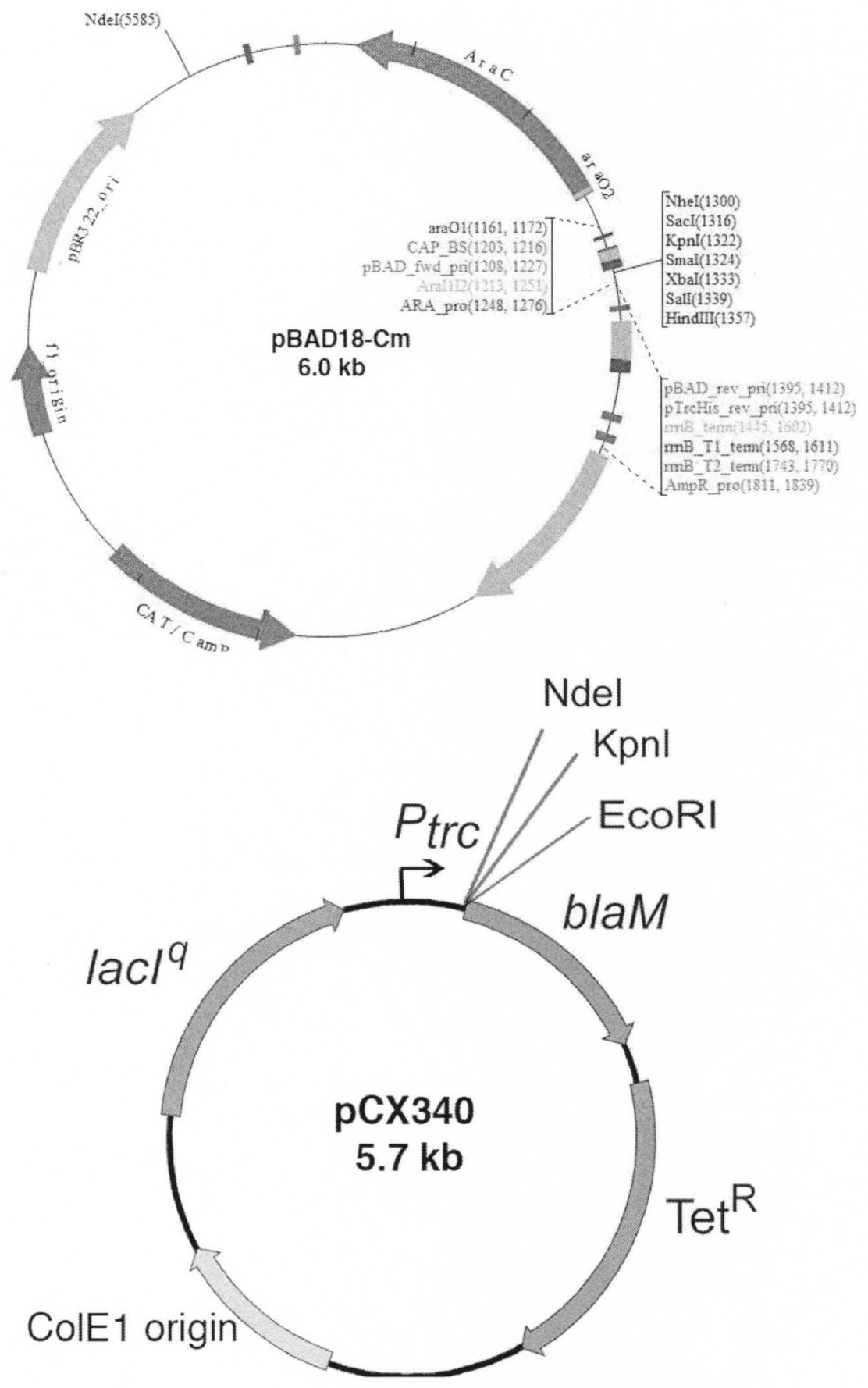

Figure 28. Diagrams of the bacterial expression vectors pBAD18-Cm and pCX340. 

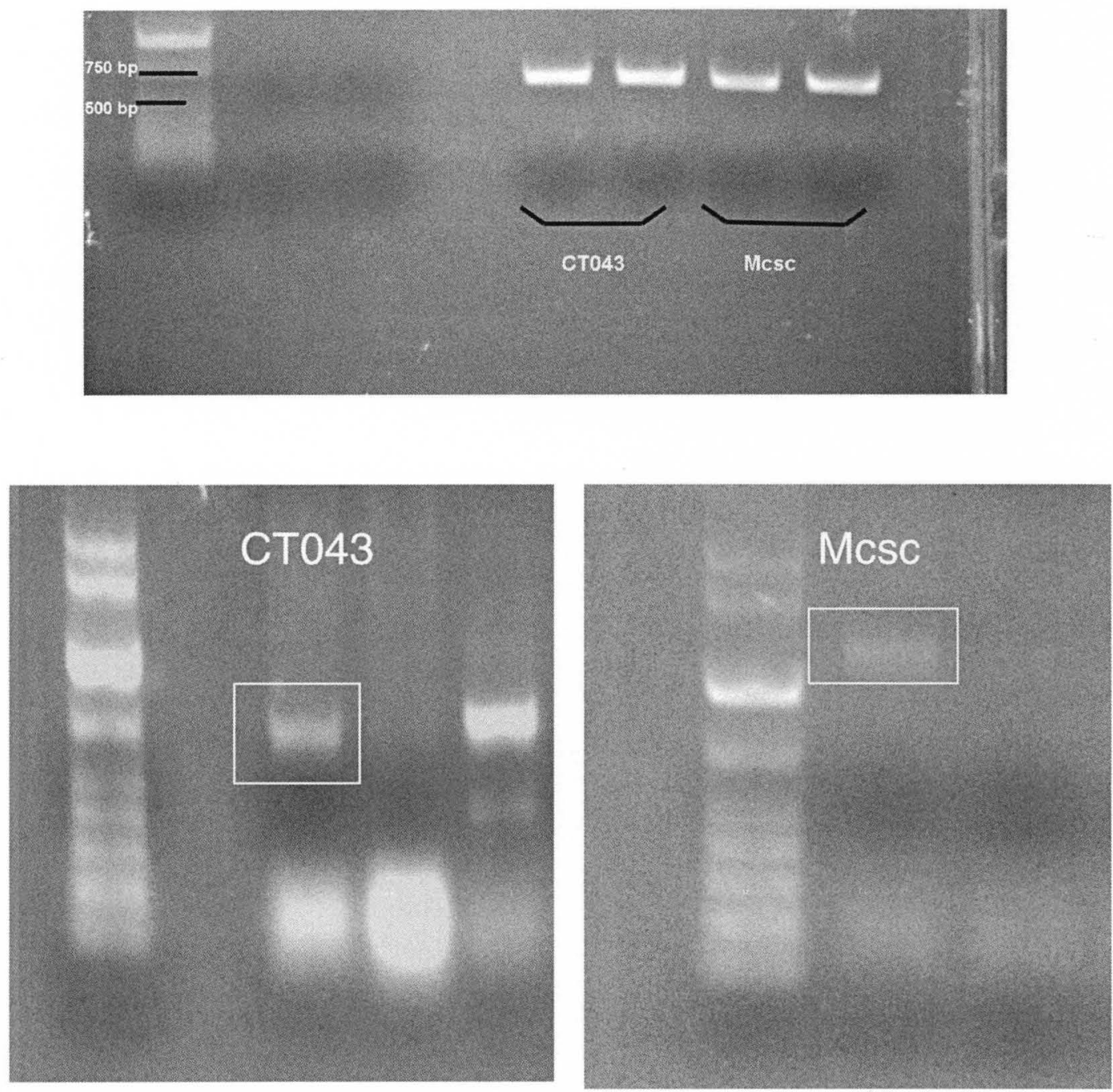

Figure 29. Cloning chlamydial chaperones into the pBAD18-Cm vector (Top) Insert PCRs of CT043 and Mcsc open-reading frames with flanking XbaI and ScaI sites. (Bottom) Colony PCRs from chloramphenicol-selected pBAD18-chaperone transformants. 

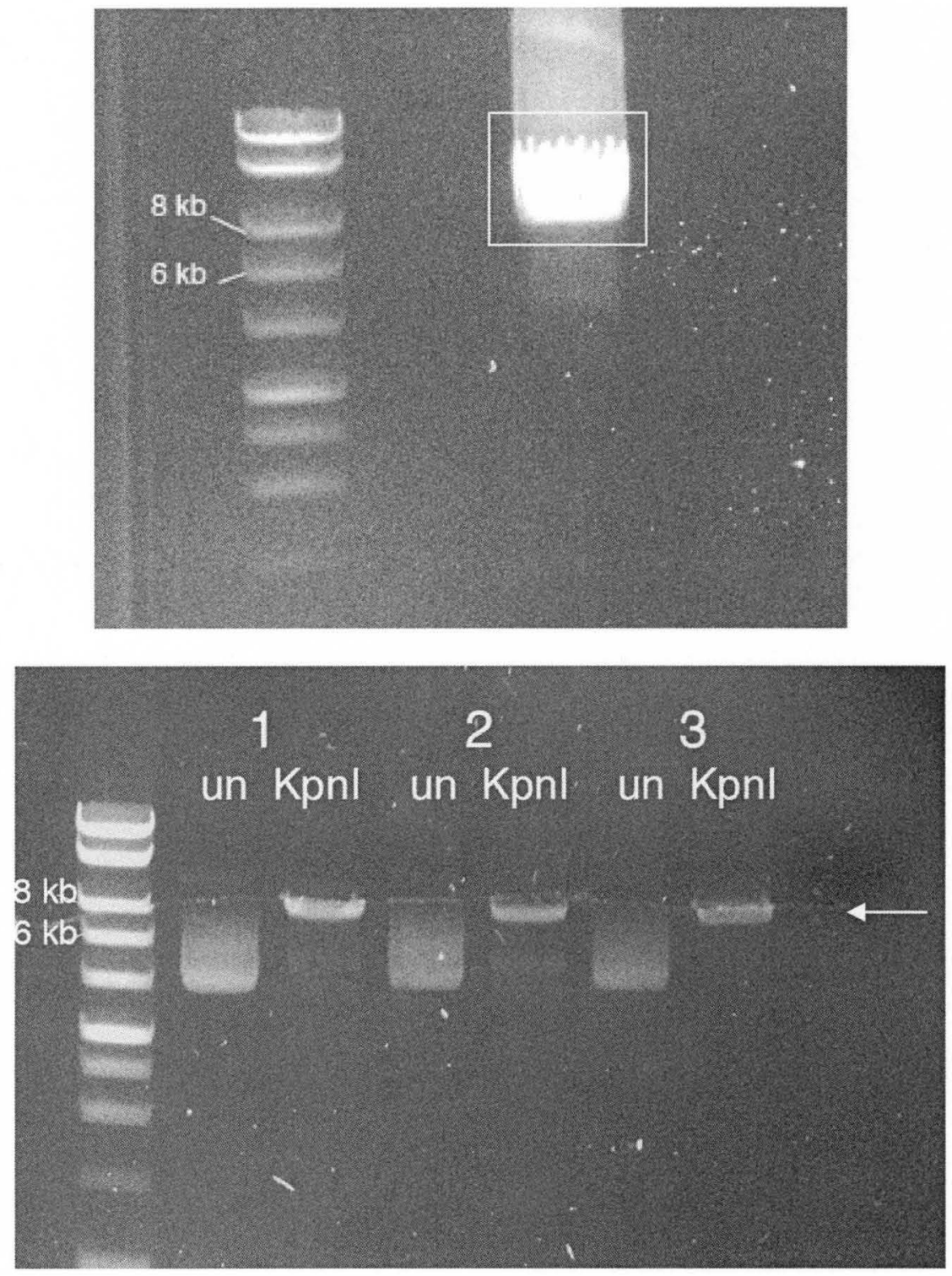

Figure 30. Adding a KpnI site to the pET200-TARP200 construct by SLIM PCR. (Top) Site-directed Lipase-Independent Mutagenesis (SLIM) based PCR was performed to add a KpnI site downstream of the TARP200 open reading frame in the pET200TARP200aa vector and the PCR product is displayed here on a $0.7 \%$ Agarose gel. (Bottom) pET200-TARP200aa-KpnI plasmid DNA prepared from kanamycin-selected B121star E.coli transformants were checked for the presence of a KpnI site by restriction digest as displayed here on a $0.7 \%$ Agarose gel. 

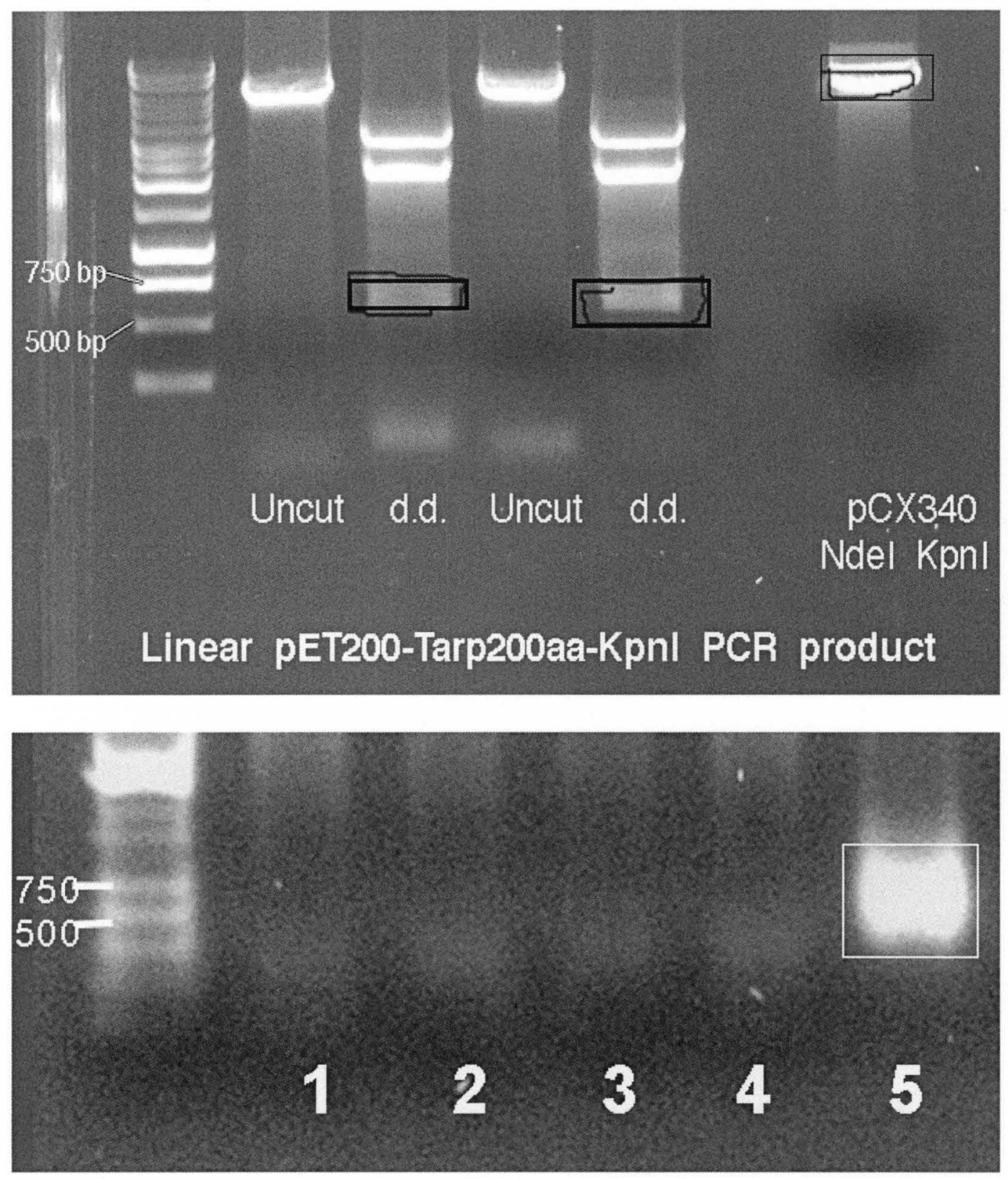

Figure 31. Cloning TARP into the pCX340 vector.

(Top) The TARP200aa open reading frame was digested with KpnI and NdeI from the pET200-TARP200 vector and pCX340 was double digested as well prior to ligation as indicated here on the $1 \%$ Agarose gel. (Bottom) Colony PCR was performed on tetracycline-selected transformants and separated on a 1.2\% Agarose gel. 
Upon contact with mammalian cells, the bacteria will translocation their type III effectors into the host cell. Treatment of the HeLa cell monolayers with CCF2/AM, a coumarin molecule bound to hexane by a $\beta$-lactam ring, following infection allows for an especially innovative fluorescence assay that takes advantage of the fact that the intact substrate fluoresces at $520 \mathrm{~nm}$ and the cleavage product, coumarin, fluoresces at $450 \mathrm{~nm}$ (after excitation at $410 \mathrm{~nm}$ ). The presence of TEM1-fused bacterial effectors in the host cell is reflected in the cleavage of the b-lactam ring to release the coumarin molecule, which I can measure its fluorescence at $450 \mathrm{~nm}$. Thus I can detect fluorescence resonance energy transfer, (FRET) of the intact molecule in the green spectrum at 520 $\mathrm{nm}$, while I can detect cleavage of the substrate as a loss of FRET in the blue spectrum at $450 \mathrm{~nm}$. A diagram of the basis of this assay is shown in Figure 32. 


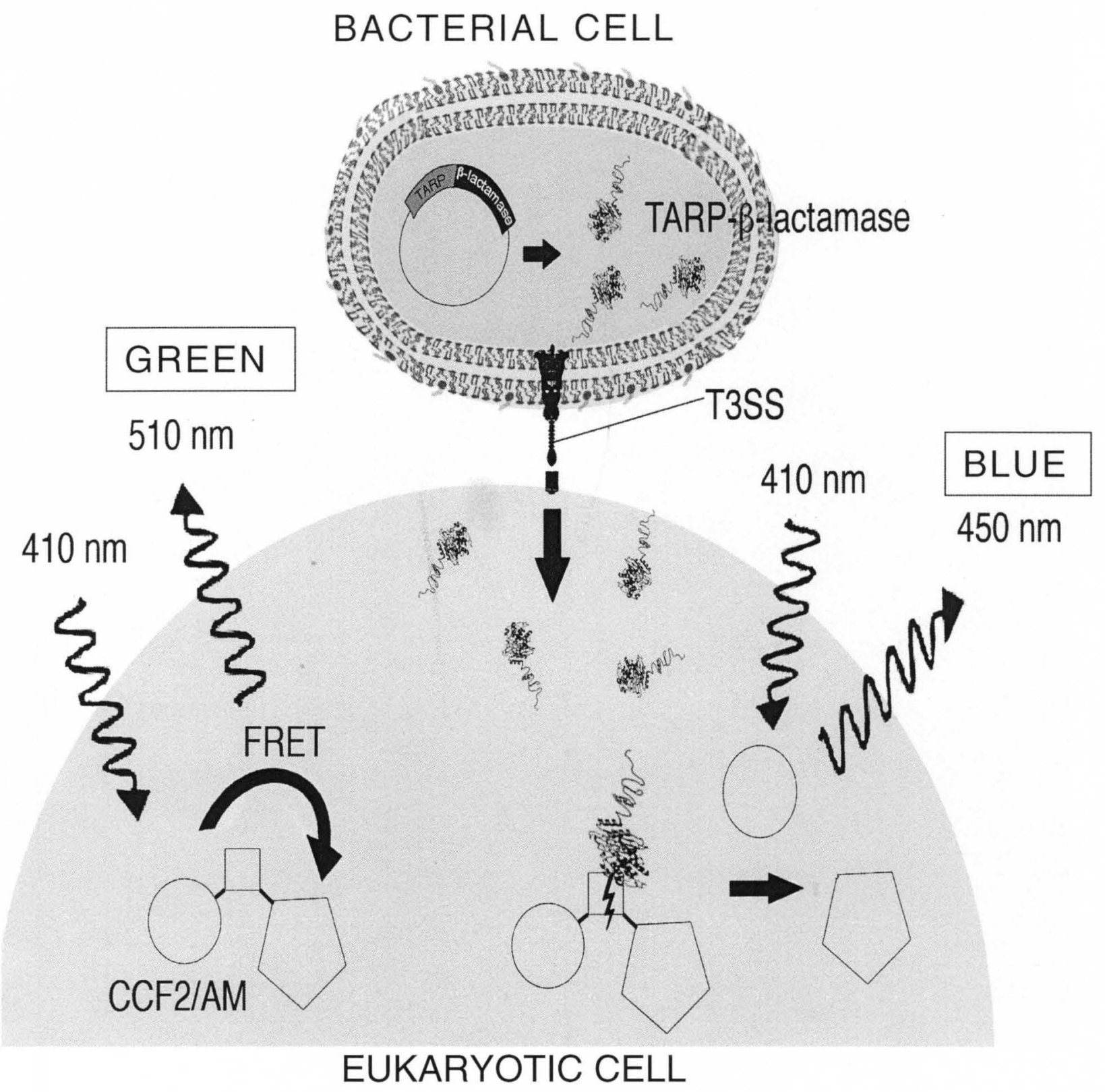

Figure 32. Diagram of the CCF2/AM fluorescence-based translocation assay In the bacterial cell, $\beta$-lactamase fused TARP is expressed from the pCX340-TARP vector. Two hours post-infection of HeLa cell monolayers, cultures are treated with CCF2/AM, a molecule that undergoes FRET (Fluorescence Resonance Energy Transfer) when excited at $410 \mathrm{~nm}$ and emits fluorescence in the green spectrum at $450 \mathrm{~nm}$. Translocation of $\beta$-lactamase fused TARP by the bacterial Type III Secretion System (T3SS) into the eukaryotic cell and subsequent cleavage of the $\beta$-lactam ring portion of $\mathrm{CCF} 2 / \mathrm{AM}$ results in a loss of FRET and an alternate emission of fluorescence in the blue spectrum at $450 \mathrm{~nm}$. 
The Yersinia ysc family type III secretion system is encoded on its virulence plasmid[63]. The type III secretion system from Yersinia pseudotuberculosis has been previously used to express and secrete chlamydial translocators and effectors, including TARP, into low-calcium media[38, 41, 150]. I was also able to detect TARP secretion into media by wild-type Yersinia enterocolitica similar to levels of secretion of the Yersinia effector, YopH (Figure 33).

However, Yersinia strains have not been tested for their ability to translocate chlamydial proteins into eukaryotic cells. I chose to utilize the type III secretion system of Yersinia enterocolitica to determine the role of Slc1 in the secretion and translocation of TARP. Based on previous findings, I expect that some TARP will be secreted and possibly translocated in the absence of its cognate chaperone[42]. However, our interaction studies indicate that the chaperone Slcl binds TARP in a fashion typical of export chaperones convincing us that Slcl will enhance TARP's interaction with the type III secretion apparatus and thus increase the amount TARP secreted or translocated by the Yersinia enterocolitica type III secretion system.

Wild type and type III ATPase (YscN) knockout strains of Y. enterocolitica expressing $\mathrm{TARP}+\mathrm{Slc} 1$ were allowed to infect HeLa cell monolayer for 2 hours prior to CCF2/AM treatment. A loss of FRET ( $\beta$-lactamase-mediated cleavage) to FRET (no cleavage) ratio was used to detect translocation. Translocation of the positive control, YopH was detected at a fluorescence ratio of $\sim 0.8$, and the negative control empty vector indicated at ratio of less than 0.1 (Figure 34). I was able to detect Slc1-mediated translocation of TARP as indicated by the ratio of 0.67 by co-expressing bacteria and 0.43 from TARPonly expressing bacteria. Because the YscN knockout strains were unable to translocate 
any effectors, I can be absolutely positive they are being translocated through the Y.enterocolitica type III secretion needle.

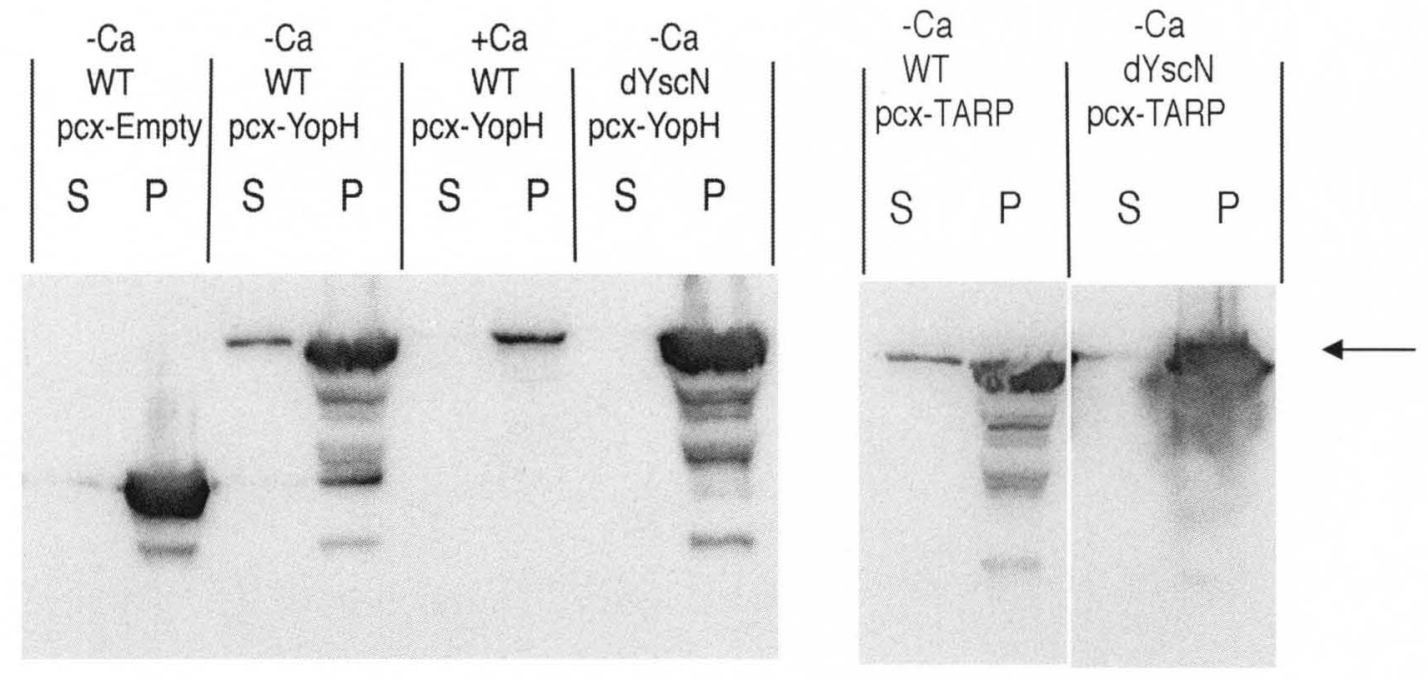

Figure 33. Secretion of HIS $_{6}$-TARP ${ }^{1-200}$ by the Yersinia enterocolitica type III secretion system. Immunoblots of TCA-precipitated Yersinia enterocolitica culture supernatants (S) and whole cell pellets (P) under type III secretion inducing conditions ($\mathrm{Ca})$ or repressing conditions $(+\mathrm{Ca})$ were probed with $\beta$-lactamase $\mathrm{mAb}$. Secretion was tested by wild-type (WT) or type III secretion knockout ( $\mathrm{dYscN}$ ) Yersinia enterocolitica strains. 


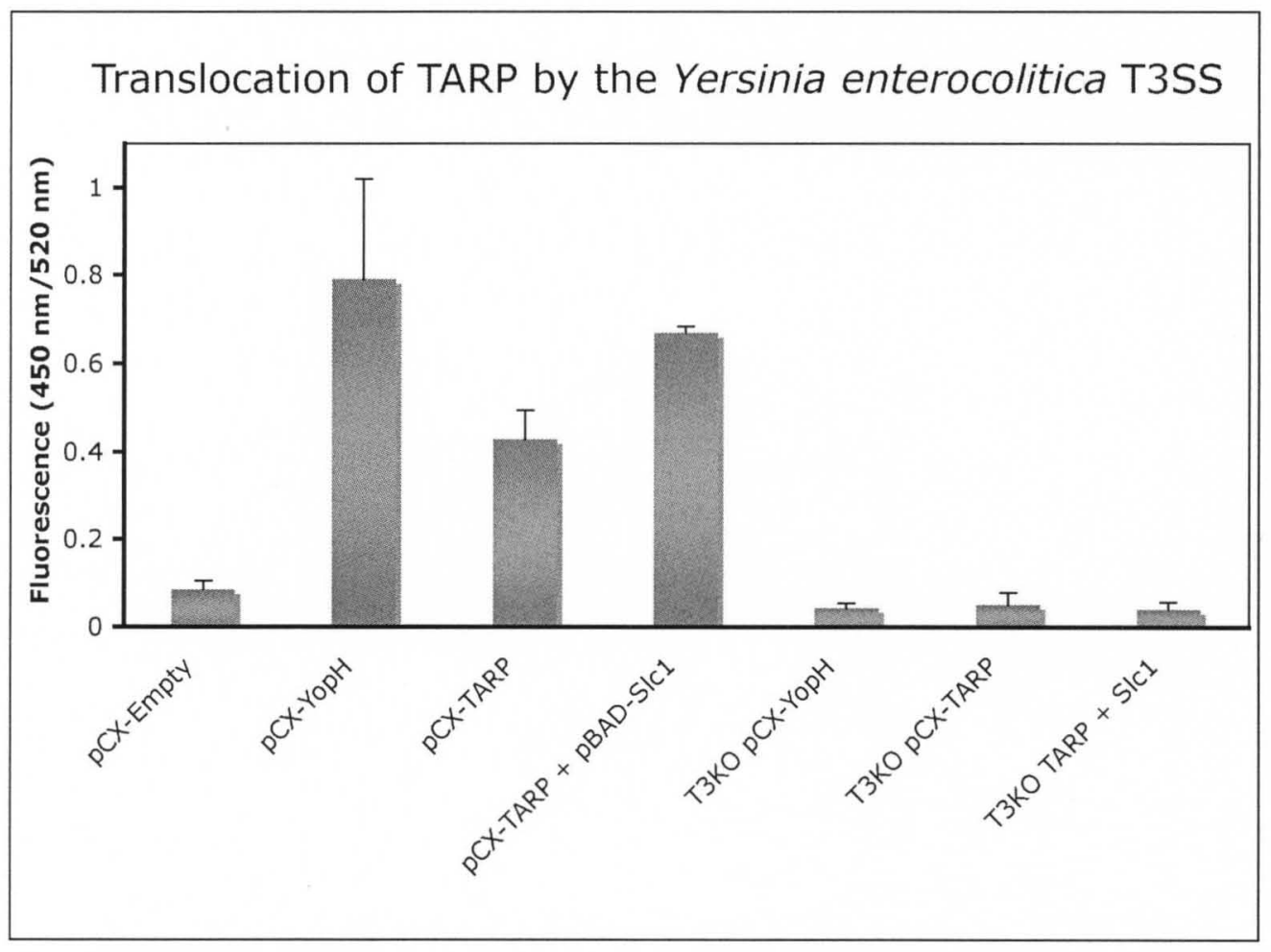

Figure 34. Translocation of TARP by Yersinia enterocolitica.

The fluorescence ratio of cleaved CCF2/AM substrate $(450 \mathrm{~nm})$ to uncleaved substrate $(520 \mathrm{~nm})$ indicates the relative translocation of $\beta$-lactamase fused effectors by the ysc type III secretion system of $Y$. enterocolitica. Wild-type (WT) and the Type III ATPase $y s c N$ knock-out (T3KO) strains bearing the $\beta$-lactamase fusion vector pcx340 with or without pBAD-Slc1 were allowed to infect HeLa cells prior to treatment with CCF2/AM substrate and fluorescence detection. 
I next used the Enteropathogenic Escherichia coli (EPEC) to test the translocation of TARP. To date there has been no evidence of secretion of chlamydial proteins by the EPEC type III secretion of TARP. However, EPEC is known to translocate the type III effector Tir that has very similar function in vivo as TARP. They both induce massive cytoskeleton rearrangement to ensure close attachment and survival of the bacteria. Furthermore alignment of type III secretion chaperones has revealed that Tir's cognate chaperone CesT has very similar secondary structure as Slc1. Therefore I thought it likely that the EPEC type III secretion could translocate TARP in the presence of its cognate chaperone Slc1.

EPEC attaches to epithelial cells and begins to translocate its effectors as soon as 30 minutes post-infection. I was able to detect translocation of the EPEC effector EspH as a fluorescence ratio of 1.5 after 1 hour of infection. Using the same infection conditions I was unable to detect any chaperone-dependent or -independent translocation of TARP. (Figure 35), and lengthening the duration of infection did not change this negative result (data not shown). 


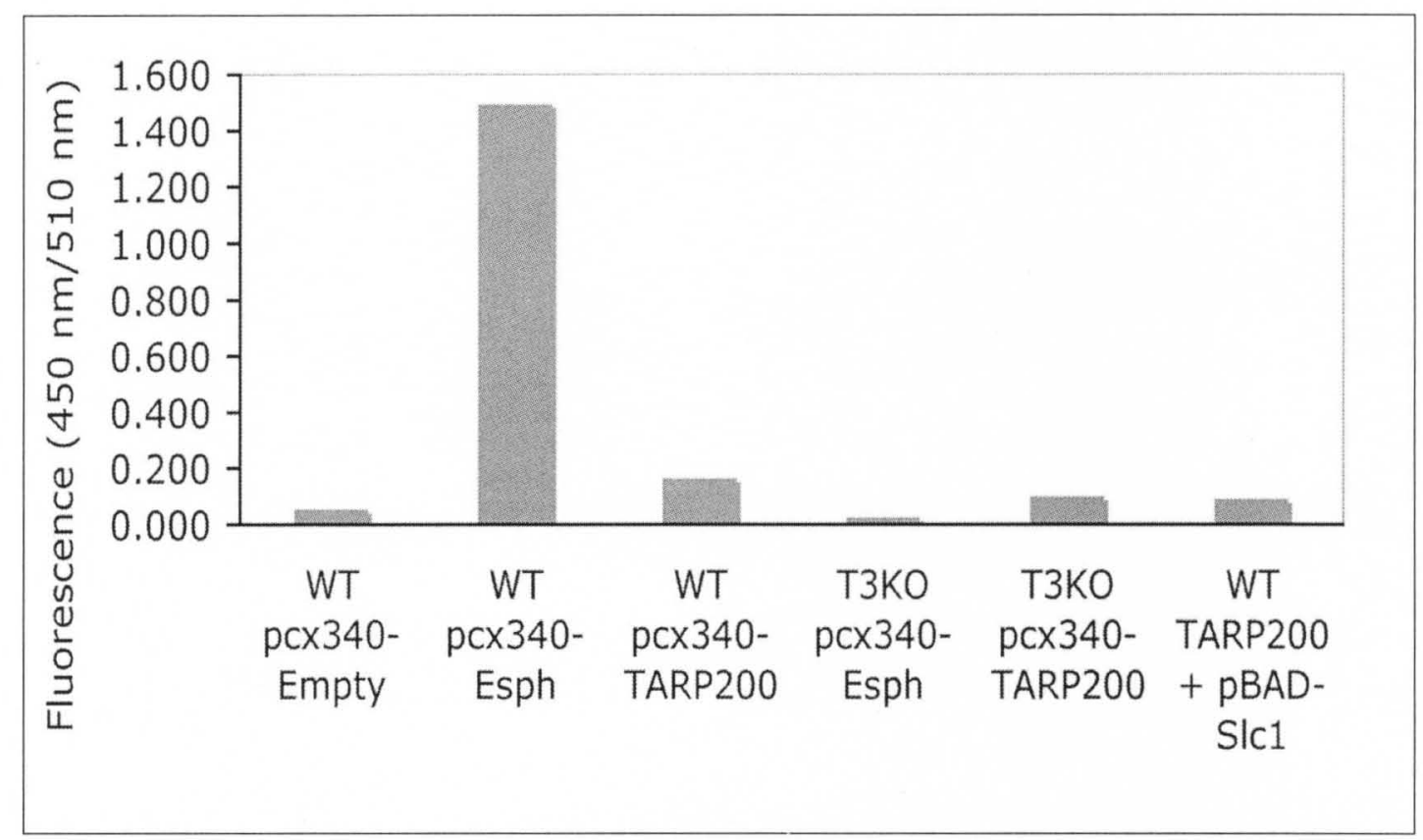

Fig 35. Translocation of TARP by Enteropathogenic $E$. coli

The fluorescence ratio of cleaved CCF2/AM substrate $(450 \mathrm{~nm})$ to uncleaved substrate $(520 \mathrm{~nm})$ indicates the relative translocation of $\beta$-lactamase fused effectors by the type III secretion system of Enteropathogenic E. coli (EPEC). Wild-type (WT) and the Type III ATPase $e s c N$ knock-out (T3KO) EPEC strains bearing the $\beta$-lactamase fusion vector pcx340 with or without pBAD-Slc1 were allowed to infect HeLa cells prior to treatment with CCF2/AM substrate and fluorescence detection. 
Salmonella typhimurium contains two functional type III secretion systems that are temporally regulated and are responsible for translocating distinct sets of effectors into epithelial cells. The SPI-1 (Salmonella pathogenicity island-1) type III secretion system translocated effectors upon contact with host cells to induce uptake of Salmonella into endocytic vesicles. After complete engulfment of the bacterium, it switches to secretion of effectors involved in maintaining its Salmonella-containing vesicle (SCV) and in redirecting host cell vesicle trafficking to ensure its intracellular survival[63]. Because the SPI-1 T3SS is responsible for secreting proteins involved in invasion of eukaryotic cells, it is an attractive choice for testing TARPs translocation. However, the SPI-2 system resembles that of Chlamydia because of its ability to secrete from an intracellular vesicle indicating that it also is worth trying as a heterologous secretion system.

As indicated by the fluorescence ratios of infected monolayers, the Salmonella SPI-1 secretion system was unable to translocate TARP under any conditions (Figure 36), but the SPI-2 system seemed to minimally translocate TARP in a chaperoneindependent manner, but achieved efficient translocation of TARP in the presence of its cognate chaperone Slc1 (Fig 34). However the high ratios for the $\beta$-lactamase only (pcx340-Empty) translocation casts some doubt on the reliability of this assay under SPI2 conditions. Furthermore, this assay has remained untested for translocation during intracellular growth (8-12 hours post-infection); instead its use has been for detection of translocation of substrates immediately after attachment (1-3 hours post-infection). 


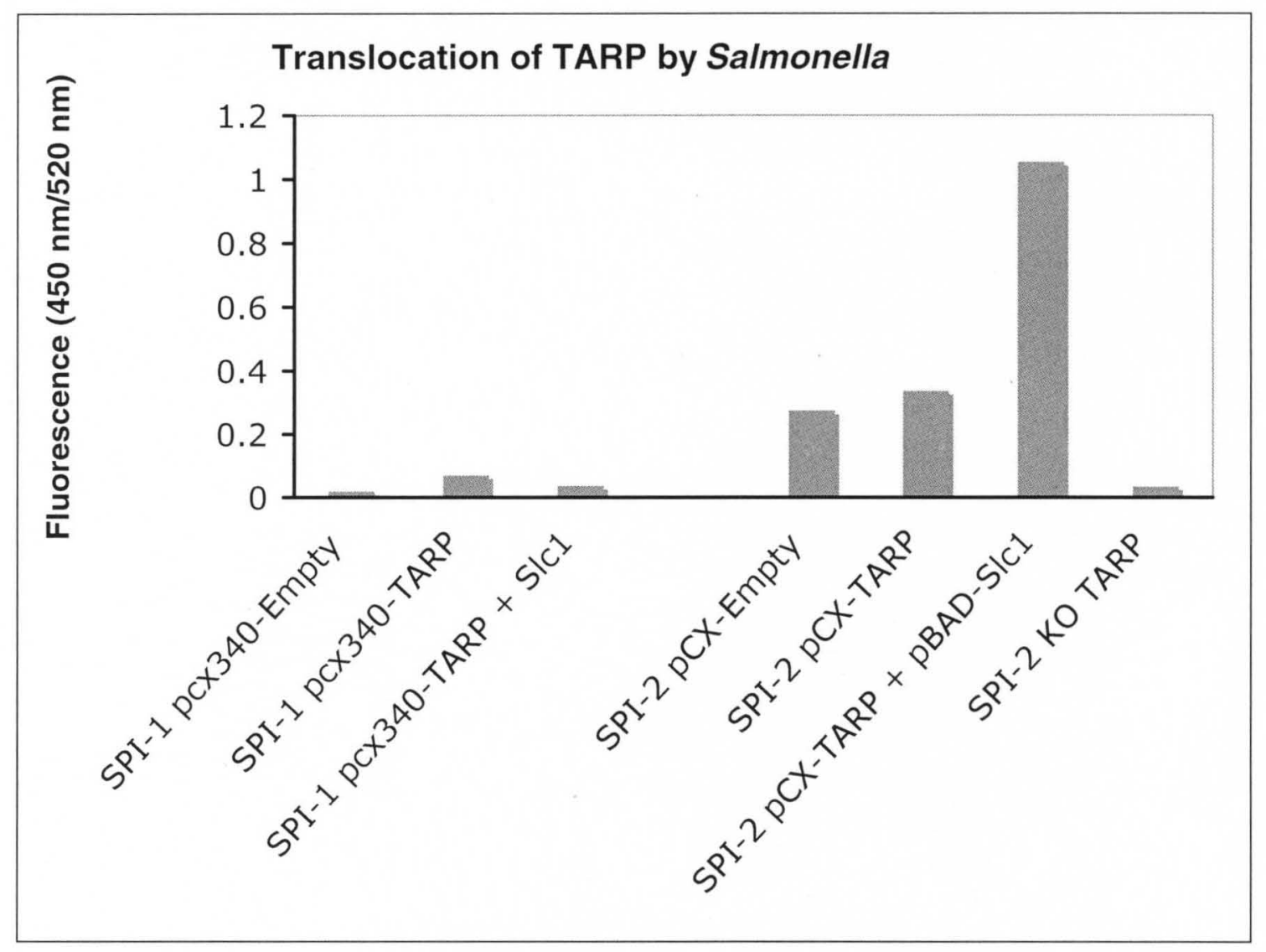

Figure 36. Translocation of TARP by Salmonella typhimurium SPI-1 and SPI-2

The fluorescence ratio of cleaved CCF2/AM substrate $(450 \mathrm{~nm})$ to uncleaved substrate $(520 \mathrm{~nm})$ indicates the relative translocation of $\beta$-lactamase fused effectors by the SPI-1 and SPI-2 type III secretion systems of Salmonella typhimurium. Wild-type (WT) Salmonella strains bearing the $\beta$-lactamase fusion vector pcx340 with or without Slc1 were allowed to infect HeLa cells prior to treatment with CCF2/AM substrate and fluorescence detection. SPI-1 mediated translocation was detected 2 hours post-infection, while SPI-2 mediated translocation was detected $10 \mathrm{hrs}$ post-infection. 
The differences in TARP translocation by different heterologous type III secretion systems is likely to due to the inability of the cytoplasmic components from other type III secretion systems to recognize the TARP-Slcl complex. To gain insight into this possibility, I analyzed the relatedness of the chlamydial type III ATPases CdsN and FliI with the type III ATPases from Salmonella, Yersinia, and EPEC. The amino acid sequences of these paralogs were aligned with ClustalW and the resulting alignments were organized into unrooted dendograms (Figure 37). The $\mathrm{CdsN}$ tree indicates that Yersinia type III ATPase YscN and the SPI-2 ATPase SsaN are similar to the nonflagellar chlamydial ATPase, while the ATPases from the SPI-1 and EPEC are less closely related, supporting our findings that Yersinia type III system and the Salmonella SPI-2 system could both recognize the TARP: chaperone complex and subsequently translocate TARP into HeLa cells. Furthermore, phylogenetic analysis indicates that the chlamydial flagellar type III ATPase FliI is more similar to the EPEC and SPI-1 nonflagellar ATPases than to relevant ATPases in Y.enterocolitica and the Salmonella SPI-2 systems. 


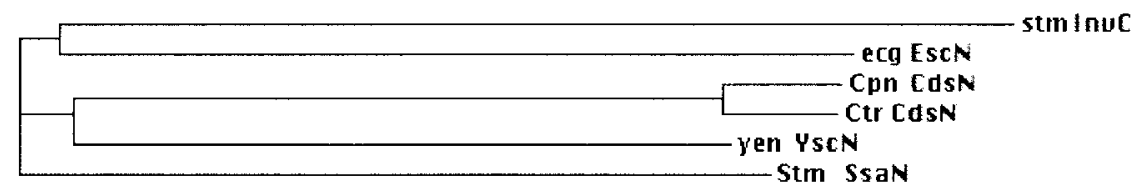

CdsN

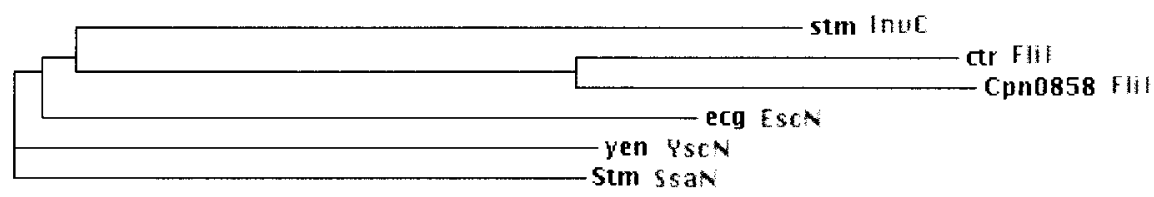

Flil

Figure 37. Phylogenetic analysis of multiple type III components from bacterial pathogens.

Unrooted phylograms were created from ClustalW-generated multiple sequence alignments of the C. pneumoniae (cpn) and C.trachomatis (ctr) ATPases CdsN (top) and Flil (bottom) with the relevant ATPases from S. typhimurium (stm), and Enterropathogenic E.coli (ecg). Orthologs were identified for comparison by the Kyoto Encyclopedia of Genes and Genomes (KEGG). 
TABLE 5. STRAINS AND PLASMIDS USED IN THIS STUDY

Strains

Top 10 (DH10B)

BL21(DE3 $\lambda$ )

DHM 1

E2348/69

ICC192

$12023 \mathrm{~s}$

HH109

MRS40(pYV40)

MRS40(pMSL41)
Description

E.coli high-level propagation

E.coli high-level expression

Adenylate cyclase deficient E.coli

Wild type EPEC 0127:H6

EPEC E2348/69 $\triangle \mathrm{escN}$

Wild type Salmonella enterica typhimurium ssaV::aphT in 12023 (Kmr)

Yersinia enterocolitica, ampicillin-resistant derivative

YscN ATPase knockout of MRS40

\section{Plasmids}

pEGFP-TARP

N-MCherry-

pDEST

pMch-Slc1

pMch-Slc2

pMch-Scc1

pET101

pET200

pENTR-D/SD-

TOPO

pET56-DEST

pSlc1-FLAG

pSlc2-FLAG

pTARP200

p56-Scc1

p56-Slc 1

pUT18C

pKT25

p18C-TARP

p18C-Tir

p18C-CT043

p18C-CT088

p18C-CT260

p18C-CT663

p25-CT043

p25-CT088

p25-CT260

p25-CT663

p25-CesT full length C.caviae TARP in pEGFP-C1 vector

pCDNA 6.2 derived vector with $\mathrm{N}$-terminal Mcherry

N-Mcherry-pDEST expressing Slc1

N-mcherry-pDEST expressing Slc2

$\mathrm{N}$-mcherry-pDEST expressing Scc 1

directional TOPO expression vector

directional TOPO expression vector

directional TOPO entry vector for LR recombination

bacterial expression destination vector (LR)

pET101 expressing Slc1-FLAG

pET101 expressing Slc2-FLAG

pET200 expressing N-term 200aa of L2 TARP

pDEST-56 expressing Scc1-FLAG

pDEST-56 expressing Slc1

E.coli 2-hybrid vector - cya fragment 18 fusion

E.coli 2-hybrid vector - cya fragment 25 fusion

E2H -cya-1 8 fragment fused to Nterm 200aa TARP

E2H -cya-18 fragment fused to Nterm 200aa Tir

E2H -cya-18 fragment fused to CT043

$\mathrm{E} 2 \mathrm{H}$-cya-1 8 fragment fused to CT088

E2H -cya-18 fragment fused to CT260

E2H -cya-18 fragment fused to CT663

E2H -cya-25 fragment fused to CT043

E2H -cya-25 fragment fused to CT088

E2H -cya-25 fragment fused to CT260

E2H -cya-25 fragment fused to CT663

E2H -cya-25 fragment fused to CesT

\section{Reference}

Life Technologies Life Technologies

Karimova,G (1995)

Levine et al (1978)

Garmendia et al

(2004)

NCTC

Deiwick et al.(1999)

Sory,M.P. (1994)

Sory,M.P. (1995)

Lane et al, (2008)

Scott Grieshaber

This study

This study

This study

Invitrogen

Invitrogen

Invitrogen

Novagen

This study

This study

This study

This study

This study

Karimova,G (1998)

Karimova,G (1998)

This study

This study

This study

This study

This study

This study

This study

This study

This study

This study

This study 
pCX340

pBAD-TOPO

pBAD18

pCX340-

TARP200

pCX340-PipB2

pBAD-CT043

pBAD-CT088

pBAD-CT260

pBAD-CT663
TEM1 fusion, IPTG inducible

Arabinose-inducible promoter

Arabinose-inducible promoter

N-term 200aa of TARP fused to TEM1

$\mathrm{N}$-term 200aa of PipB2 fused to TEM1

pBAD-TOPO with CT043-FLAG insert

pBAD18 with CT088-FLAG insert

pBAD-TOPO with CT260-FLAG insert

pBAD18 with CT663-FLAG insert
Charpentier (2004)

Invitrogen

Guzman (1995)

This study

This study

This study

This study

This study

This study

TABLE 5. PRIMERS USED IN THIS STUDY

$\begin{array}{ll}\text { Primers } & \text { Sequence 5' } \Rightarrow \text { 3' } \\ \text { AJB1F } & \text { CACCATGTCCAGGCAGAATGCTGA } \\ \text { AJB1R } & \text { TCACTTGTCATCGTCGTCCTTGTAGTAGCACGGATTCCTGCGGTA } \\ \text { AJB2F } & \text { CACCATGTTGGAAAAATTGATAAAGAATTTGTGGCG } \\ \text { AJB2R } & \text { TTACTCCTGCTCCGTTTTACTTAGTCTAAATC } \\ \text { AJB3F } & \text { CACCATGCAAAATCAATTTGAACAACTC } \\ \text { AJB3R } & \text { ATCGATTTACTTGTCGTCATCGTCCTTGTAGTCCAGGTGATACAT } \\ \text { AJB4F } & \text { CACCATGACGAATTCTATATCAGGTGATC } \\ \text { AJB4R } & \text { TTATCCTACGGTATCAATCAGTGAGCT } \\ \text { AJB5R } & \text { TTATGCACGGATTCCTGTGGAGG } \\ \text { AJB6FT } & \text { CCTTCACCAAGGGTACCTCAACGATCCGGCTGCTAACAAAGCC } \\ \text { AJB6FS } & \text { CCGGCTGCTAACAAAGCC } \\ \text { AJB6RT } & \text { TCGTTGAGGTACCCTTGGAGGAGCCTCTTAGAGA } \\ \text { AJB6RS } & \text { CTTGGAGGAGCCTCTTAGAGA } \\ \text { AJB7FT } & \text { CGATTAAATAAGGAGGAATAACATATGACGAATTCTATATCAGGTGATC } \\ \text { AJB7FS } & \text { ACGAATTCTATATCAGGTGATCAACCTA } \\ \text { AJB7RT } & \text { CATATGTTATTCCTCCTTATTTAATCGCGATACATTAATATATACCTCT } \\ \text { AJB7RS } & \text { CGATACATTAATATATACCTCTTTAATTTTTAATAATAAAG } \\ \text { AJB8F } & \text { ACCATGGCCTCCAGGCAGAATGCT } \\ \text { AJB8R } & \text { TTATTAGTCCTTATCGTCATCGTCCTTGTAATCTGCACGGATTCTGCT } \\ \text { AJB10F } & \text { ACCATGGCCACAACGTGGACTTTGAATCAC } \\ \text { AJB10R } & \text { TTATTAGTCCTTATCGTCATCGTCCTTGTAATCAGGCTCTAGCTGATCGGAT } \\ \text { AJB13R } & \text { GAACTAACGTATGGAGAAAGGTACCCAAGCTTCCAGCAACGGCTT } \\ \text { AJB14F } & \text { CGTAGGACGCATATGAACTTATCATTAAGCGAT } \\ \text { AJB14R } & \text { TGAATCTCAGGTACCGCGGCTGCTGAGTTC } \\ \text { AJB15F } & \text { TAGCATCGGGTACCGATGACGAATTCTATATCAG } \\ \text { AJB15R } & \text { CGTAGCTATCGATGAGCCACTTGTGCTAGA } \\ \text { AJB16F } & \text { GTTACGATCTAGAGATGCCTATTGGTAACCTTGGTAATA } \\ \text { AJB16R } & \text { CTAGTGCAGGTACCTTACGTATCAACGTCCTT } \\ \text { AJB17F } & \text { TTACGTATTCTAGAGATGTCCAGGCAGAATGCT } \\ \text { AJB17R } & \text { GCATTAGTGGTACCCTTATGCACGGATTCCTGC } \\ \text { AJB18F } & \text { TGTACCGTATTCTAGAGATGCAAAATCAATTTGAAC } \\ \text { AJB18R } & \text { TGCTATCGGTACCTTACAGGTGATACATACCT } \\ \text { AJB19F } & \text { TTACGTATTCTAGAGATGACAACGTGGACTTTG } \\ & \end{array}$




$\begin{array}{ll}\text { AJB19R } & \text { GCATTAGTGGTACCCCTAAGGCTCTAGCTGATC } \\ \text { AJB20F } & \text { GGACGGACTCTAGAGATGTTGGAAAAATTGATA } \\ \text { AJB20R } & \text { GCATTAGTGGTACCCTTACTCCTGCTGCGTTTT } \\ \text { AJB21F } & \text { TAACAGTAGGTACCTTATCTTCCGGCGTA } \\ \text { AJB21R } & \text { TTACGTATTCTAGAGATGTCATCAAGATCTGAAC } \\ \text { AJB22F } & \text { CATTATTCTAGAGATGTCCAGGCAGAATGCTGAG } \\ \text { AJB22R } & \text { TTGCTAGTACCCGTTATGCACGGATTCCTGCTGG } \\ \text { AJB23F } & \text { CATTATTCTAGAGATGCAAAATCAATTTGAACAA } \\ \text { AJB23R } & \text { TTGCTAGTACCCGTTACAGGTGATACATACCTAG } \\ \text { AJB24F } & \text { CATTATTCTAGAGATGACAACGTGGACTTTGAAT } \\ \text { AJB24R } & \text { TTGCTAGTACCCGTTAAGGCTCTAGCTGATCGGA } \\ \text { AJB25F } & \text { CATTATTCTAGAGATGTTGGAAAAATTGATAAAG } \\ \text { AJB25R } & \text { TTGCTAGTACCCGTTACTCCTGCTCCGTTTTCTT }\end{array}$




\section{DISCUSSION}

Many intracellular bacterial pathogens use specialized secretion systems to translocate effectors into eukaryotic cells and in turn these effectors recruit, modify, or hijack cellular components to the benefit of the bacterium. Effectors of bacterial type III secretion systems have been reported to be involved in remodeling the eukaryotic cytoskeleton, altering the immune response, hijacking trafficking vesicles, disrupting cell-to-cell junctions, and causing cytotoxicity[63].

Survival and pathogenesis of Chlamydia spp. is entirely dependent on its ability to invade mammalian epithelial cells and develop a unique niche that is conducive to intracellular growth and replication. Translocation of effectors by a type III apparatus is a major strategy for Chlamydia entry, inclusion formation, and survival in eukaryotic cells $[46,84,85]$. Recruitment of host kinases and actin, in addition to activation of Rac and actin-poylmerization aid in endocytosis, and are functions attributed to the type III invasion associated effector TARP[42, 66, 67, 69, 132]. Chlamydia trachomatis urogenital, lymphogranuloma, and ocular strains synthesize TARP homologs that contain three functional domains: tyrosine-rich repeats for recruitment of host kinases and subsequent Rac activation, a proline-rich aggregating domain, and actin-nucleating WH2like domains. 
Detected and tyrosine-phosphorylated in host cells within minutes after cell contact, TARP translocation is essential for the efficient uptake of C. trachomatis EBs. TARP homologs in C.pneumoniae, C.caviae, and C.psittaci do not contain tyrosine-rich repeat domains, but they are still able to invade host cells. In addition the WH2-like domains are sufficient to nucleate actin in host cells, which are also important for infection $[70,71]$. This redundancy between TARP domains indicates another possible function for C.trachomatis TARP tyrosine-phosphorylation during chlamydial infection. It has been suggested that the tyrosine phosphorylation may help ensure specific uptake pathways that prevent phagolysosomal fusion. This theory has been supported by the detection of TARP localized to the inclusion throughout the developmental cycle[42].

Given TARP's pivotal roles in entry and intracellular survival, I considered possible type III secretion chaperones that would increase the efficiency of TARP translocation into host cells. I determined that the chlamydial chaperone Slc1 specifically interacts with TARP and mediates its translocation by the heterologous type III secretion system of Yersinia enterocolitica. Identification of putative type III related genes in bioinformatics studies identified three putative chlamydial effector chaperones: Slc1, Slc2, and Scc1. Since Class I effector chaperones share the specific secondary structure $\alpha-\beta-\beta-\beta-\alpha-\beta-\beta$, putative chlamydial chaperones were readily identified by in silico approaches. Detection of these three proteins in elementary bodies indicated that any one of them could be involved in maintaining TARP in a secretion-competent state for immediate translocation into host cells. 
In other gram-negative bacteria, previously identified type III Class I effector chaperones demonstrated binding of their effectors adjacent to their $\mathrm{N}$-terminal secretion signal, usually within the $\mathrm{N}$-terminal 30-130 amino acid range.

\section{Identification of the TARP's cognate secretion chaperone SIc1}

I used a direct co-immunoprecipitation approach for identifying a chlamydial chaperone that could interact with the $\mathrm{N}$-terminal 200 amino acids of C.trachomatis L2 TARP. I was specifically able to co-immunoprecipitate Slc1 with TARP in two independent co-IP approaches. Separate expression of Slc1 and TARP in E.coli prior to incubation of their lysates with M2 Anti-FLAG agarose yielded an interaction between Slcl and TARP, but co-expression of these proteins prior to co-immunoprecipitation greatly increased their ability to interact. This indicates to us that Slc1 may mediate folding of TARP or prevent its pre-mature interaction with other E.coli components when expressed in the same bacterium. However, neither co-immunoprecipitation technique was able to demonstrate an interaction between TARP and the other putative chlamydial chaperones Slc2 or Scc1.

One other explanation for the increased interaction between Slcl and TARP during co-expresssion would be that Slc1 prevents TARPs degradation or solubility. The cognate chaperone SycE in Yersinia helps to solubilize its cognate aggregrate-proned effector YopE[151], and similarly the EPEC chaperone CesT prevents the degradation of the effector Tir[152]. Despite the described ability of many known type III chaperones to solubilize and stabilize their effectors, I was not able to detect any changes in solubility 
or stability of TARP when expressed in the presence or absence of its chaperone (data not shown).

One caveat of detecting chaperone: TARP interactions in E.coli lysates, is that an E.coli protein may be mediating the interaction detected between Slcl and TARP. It is difficult to refute this claim, because attempts to find an interaction between pre-purified TARP and Slc 1 were unsuccessful. Because this possible E.coli factor did not mediate an interaction between TARP and Scc1 or Slc2, which are predicted to have extremely similar secondary and tertiary structures as Slc1, I propose that it is highly unlikely for an E.coli factor to be so specific for Slcl and TARP.

Our co-immunoprecipitation findings were confirmed by the specific interaction detected between Slc1 and TARP in an adenylate-cyclase (cya) based bacterial 2-hybrid system[148]. Because the readout for interaction of cya-fused target and bait proteins in this system was the cAMP-mediated up-regulation of the lactose and maltose operons, I was able to detect interactions in a variety of assays. Both X-gal and McConkey plates indicated a range of protein-protein interactions from low-affinity (chaperone-effector) to high affinity (leucine-zipper proteins). While the indicator plates allowed for obvious qualitative results of chaperone-effector interactions, they were not able to differentiate between slow and fast growing strains. If allowed to grow long enough, even the negative control strains would begin to change color on these indicator plates, therefore I had concerns that any slow-growing strains would not indicate obvious interactions.

To alleviate this problem as well as to quantitatively compare protein-protein interactions, I employed the $\beta$-galactosidase assay which not only takes into account the amount and time of ONPG conversion, but also the total number of bacteria in each 
sample. Compared to co-expression with other chaperones tested in this assay, DHM1 E.coli co-expressing Slc1 and TARP converted 2-3 times as much ONPG to the colorimetric substrate ONP, confirming the bacterial 2-hybrid results on indicator plates.

\section{Characterizing all possible chlamydial chaperone-chaperone interactions}

The tendency of type III chaperones to dimerize prior to interacting with their effectors, led us to detect whether Slc1 tends to form homodimers or heterodimers. From co-immunoprecipitation assays I was able detect Slc1 interactions with itself, but not with the other putative chaperones Slc2 or Scc1. Since I singly expressed the chaperones prior to adding the lysates to the M2 Anti-FLAG agarose or S-rek resin, it is likely that Slc1 dimers were formed early, leaving very little monomeric Slc1 to interact with other chaperones. Instead of testing co-expression in the co-IP assays, I thought it best to test co-expressed chaperone-chaperone interactions in the bacterial 2-hybrid system. In this system I was able to confirm previous chlamydial chaperone-chaperone interactions as well as confirm our Slc1-Slc1 interaction. Obvious interactions on indicator plates included Slc1-Slc1, Mcsc-Mcsc, and Slc2-Scc1. However, I was not able to detect the reverse $\mathrm{Cya}_{18}-\mathrm{Scc} 1 / \mathrm{Cya}_{25}$-Slc2 interaction indicating that the dimer formed may only allow interaction of the 18 and 25 fragments when the dimer is formed in the $\mathrm{Cya}_{18}$-Slc2/

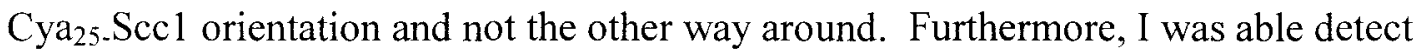
expression of both 18-Scc1 and 25-Slc2; consequently the lack of bidirectional interaction was not due to the constructs being problematic.

Quantitative analysis of chaperone-chaperone interactions in the bacterial 2hybrid strains indicated the same positive interactions as on indicator plates, but there 
also seemed to be a low level of interaction between most of the chaperones. The Slc1Slc1 interaction was only 2-fold higher and the Slc2-Scc1 interaction was only slightly higher than most of the chaperone-chaperone strains. I speculate that Chlamydia maintains a low level of interaction between type III class I chaperones to enable the bacterium to quickly acquire new type III effectors. This is supported by the fact that Candidata Protochlamydia ameobophilia maintains SycE-like Class I chaperones in the same gene order as in pathogenic Chalmydia, however the chaperones themselves have completely diverged in amino acid sequence[22,29]. In addition, recent studies have identified species-specific effectors among chlamydial pathogens[84], indicating a mechanism for the quick selection of new type III effectors for adaptation to new host cell types must exist. It has been noted in other bacteria that there is little conservation in sequence or structure of type III effectors, despite the requirement that all effectors must be adequately unfolded to ensure secretion through the $\sim 4 \mathrm{nM}$ diameter needle[29, 59, 153]. Thus type III chaperones may be necessary to partially unfold disordered regions in effectors so that they may effectively interact with the type III ATPase $[59,153]$.

Akeda et al. maintain that the ATPase actually does the majority of unfolding effectors in addition to providing active (ATP-dependent) separation of effectors from cognate chaperones[59]. Recent crystallizations of chaperone: effector complexes support this theory that chaperones only act to locally unfold or reorder the effector at the chaperone binding domain (CBD). Whether this localized unfolding allows for the unfolding of the $\mathrm{N}$-terminal secretion signal or makes the secretion signal more accessible to the ATPase is not clear. 
Besides being unfolded, secretion of type III effectors requires an $\mathrm{N}$-terminal secretion signal that is possibly recognized by the ATPase. However, this signal is highly divergent and efforts to predict secretion substrates based on their N-terminal amino acid composition has remained challenging. Recent studies have used machine-learning approaches that focused on the $\mathrm{N}$-terminal 25 amino acids of known type III effectors to predict the probability that a protein is in fact an effector $[25,30]$. Predicted secretion signals were mostly disordered, largely hydrophobic, often with polar-hydrophobic-polar residue patterns, and were serine and threonine-rich. One such prediction program identified 86 putative C. trachomatis effectors, 24 of which had already been experimentally confirmed to be type III effectors[30].

Considering the small number of possible chaperones, it is clear that not all of these effectors will require a chaperone for their translocation. Thus a major enigma in bacterial type III secretion is why some effectors require chaperones while others do not. In EPEC and Yersinia, a hierarchy of secretion is established in which effectors that have cognate chaperones are secreted preferentially to those without chaperones[62, 154, 155]. Since few chlamydial chaperone-effector interactions have been detected thus far, I have no indication as to whether such a hierarchy exists during infection, but it is easy to imagine how such a hierarchy could play a role in regulating Chlamydia's developmental cycle.

The recently discovered type III secretion chaperone Mcsc was not predicted to be a Class I chaperone by in silico analysis, but its association with effectors and ability to form homodimers in a yeast 2-hybrid screen[49] led us to test its ability to interact with TARP as well as its ability to form heterodimers in our bacterial 2-hybrid assays. I did 
not detect any interaction between TARP and Mcsc, but the Mcsc-Mcsc co-expressing DHM1 strain had nearly 6 fold higher $\beta$-galactosidase activity than the Slc1-Slc1 interaction and as much as 10 fold higher than other background chaperone-chaperone interactions. Mcsc is an atypical Class Ib chaperone that has been shown to have multiple effectors and also interacts with several type III C-ring components, therefore its role in Chlamydia infection may differ from the typical effector chaperones and cannot be compared directly with other Class I chaperones.

Being associated with both type III secretion effectors and cytoplasmic components of the type III apparatus, the Mcsc dimer has been speculated to play a role in co-translational secretion of effectors[49]. No evidence has been presented thus far to suggest the coupling of effector translation and non-flagellar type III secretion in Chlamydia, nor in other bacteria. However, a model of flagellar secretion coupled with translation of the FlgM mRNA transcript has been proposed in Salmonella typhimurium[156]. In addition to Mcsc's ability to interact with apparatus components, the Chlamydia non-flagellar type III components have also been shown to interact with flagellar components[157]. Thus it is possible that the Chlamydia type III secretion system is capable of unique mechanisms of regulating substrate translation and translocation.

\section{Oligomerization state of Slc1 and Slc1: TARP complexes}

Further characterization of the Slc1-Slc1 interaction was detected with crosslinking and gel filtration chromatography. While the cross-linking with DTSSP was only supposed to stabilize the already formed Slc1 homo-complexes, I contend that trimeric 
Slc1 species were induced in the presence of the cross-linker. Purified Slc1 was highly concentrated in our samples and separate molecules of Slcl were likely in close enough contact to become cross-linked. The most revealing aspect of the cross-linking assay was the finding that Slc1 homo-dimers could be detected on a non-reducing SDS-PAGE gel even without cross-linking treatment.

Gel filtration chromatography only confirmed our hunch that native Slc1 forms stable dimeric species in solution. A single native Slc1 complex migrated through the sepharose column to a position corresponding to a molecular weight of approximately 40 $\mathrm{kDa}$, which is certainly indicative of homo-dimers. However, gel filtration chromatography is sensitive to both the size and molecular weight of the sample, so I cannot pinpoint an exact molecular weight with this assay. This approach was also useful for detecting the native Slc1: TARP complex. I expected to find a complex of a combined molecular weight of $\sim 65 \mathrm{kDa}$ ( 2 chaperone molecules to $1 \mathrm{TARP}$ ), but the Slc1 and TARP-containing elution fractions corresponded to a size $>75 \mathrm{kDa}$. One reason for this larger size could be that the complex actually has a stoichiometry of $2: 2$, however this is unlikely considering the plethora of crystallization data supporting a $2: 1$ stoichiometry for type III chaperone: effector complexes. Instead I claim that the partially unfolded TARP in combination with the chaperone dimer has an extended size that caused the complex to elute earlier than expected from the sepharose column. The importance of this 2:1 stoichiometry in mediating the interaction with the type III secretion apparatus is not Ill understood, but it is certainly an area worth investigating considering both Class II and Class III chaperones also form dimers to interact with their substrates[153]. The conservation of this chaperone dimer/substrate complex among 
bacterial type III secretion systems has led to the conclusion that this complex forms a three dimensional secretion signal that directs it to the type III apparatus[151].

A three-dimensional secretion signal that could trigger recognition by the type III ATPase has been proposed multiple times, but only a recent study was able to demonstrate the role of a chaperone-mediated translocation signal. The type III effector YopE from Yersinia pseudotuberculosis was dependent on a solvent exposed patch corresponding to the chaperone-binding domain for translocation, but interestingly these solvent exposed residues were not essential for chaperone binding[158]. This finding indicated that the type III chaperone orders the chaperone-binding domain of the effector such that a second secretion signal is exposed on the effector surface. Further work with type III chaperone-effector complexes that have been previously crystallized will indicate if the chaperone-mediated solvent-exposed patch is the universal three-dimensional translocation signal.

Considering that Chlamydia has two possible type III ATPases (CdsN and FliA) that are expressed during infection, it is probable that there is a difference in effector recognition between these ATPases[35]. Whether these ATPases (or possibly combinations of cytoplasmic type III components) would be able to differentiate between $\mathrm{N}$-terminal secretion signals (S1) and/or chaperone-mediated translocation signals (S2) is yet to be determined. Thus future experiments testing the interaction of alternate chlamydial ATPases with chaperone-substrate complexes in vitro and in heterologous systems will be revealing as to the importance of different secretion signals in type III effector recognition, as well as clue us in to possible ATPase switching during actual infection. Speculation about ATPase switching may seem far-fetched when considering 
the complete lack of precedence in other known bacterial type III secretion systems. However since the chlamydial type III secretion systems have evolved in isolation from the Proteobacteria for more than 2 billion years there will almost certainly be Chlamydia-specific type III interactions and functions that are not present in the other gram-negative type III secretion systems[22].

\section{Roles for chaperone-effector interactions in developmental cycle regulation}

As mediators of translocation type III chaperones may play an important role in the regulation of the $\mathrm{EB}$ to $\mathrm{RB}$ and/or $\mathrm{RB}$ to $\mathrm{EB}$ transitions that are necessary for establishing infection and spreading to surrounding tissues. Release of an effector, such as TARP, may allow for interaction of its cognate chaperone, Slc1, with another effector, whose translocation could trigger post-invasion events. Such an ordered process could conceivably be maintained by the specificity of the effectors' chaperone binding domain for a specific chaperone dimer. CesT for instance, in EPEC, is known to act as chaperone for multiple effectors, having the highest affinity for the invasion-associated effector Tir, which is also the first of its cognate effectors to be detected in the host cell[159].

The apparent interaction of the chlamydial chaperone Slc2 (CT663) with both E.coli RNA polymerase (RNAP) and the chlamydial major sigma factor $\sigma^{66}$ indicates a possible regulatory role for type III chaperones[159]. It is tempting to speculate that the Slc2 interaction with $\sigma^{66}$ of the chlamydial RNAP holoenzyme allows for subsequent gene transcription by the alternate sigma factor $\sigma^{28}$, possibly initiating transcription of a set of genes involved in the RB to EB differentiation or maintaining the persistent RB. Both CT663 and the $\sigma^{28}$-encoding gene fliA are mid-cycle genes and therefore their 
expressed products are likely to play a role in late developmental cycle events[93]. The Slc2-Scc1 interaction detected in two-hybrid studies may sequester Slc2 and prevent it from acting as an anti-sigma factor during specific stages of infection. Determination of Sccl's cognate effector or function during infection may provide insight into how and when Slc2 could act to regulate transcription. However, this area definitely needs to be further investigated to really determine the importance of Slc2 in gene regulation.

\section{Dependence of TARP's translocation on Slcl in heterologous systems}

While our biochemical assays only serve to indicate protein-protein interactions between putative chlamydial type III chaperones and between the chaperone Slc1 and the invasion-associated effector TARP, the findings beget many possible roles for these interactions during infection. I contend that Slcl's main role is to achieve translocation of its cognate effector TARP and subsequently ensure the efficient uptake of Chlamydia by non-phagocytic eukaryotes. Using several different heterologous bacterial type III secretion systems I attempted to determine the chaperone requirement for TARP translocation. Translocation of $\beta$-lactamase fused effectors was reported by the cleavage of a lipophilic $\beta$-lactam ring-containing compound CCF2/AM. Because excitation of the cleaved and non-cleaved substrates at $410 \mathrm{~nm}$ produced emissions of alternate fluorescence wavelengths ( $510 \mathrm{~nm}$ and $450 \mathrm{~nm}$, respectively), I was able to accurately measure TARP translocation into a population of HeLa cells by a ratio of fluorescence. This CCF2/AM based translocation assay has been widely used for detecting translocation of type III and type IV secreted effectors by Shigella, Legionella, Salmonella, Yersinia, E.coli, and Pseuodomonas[160-163]. 
The reproducibility of this assay is based in the fact that total fluorescence is measured from a population of infected HeLa cells, so as long the multiplicities of infection are equal among compared samples, then the differences in substrate absorption and number of cells in each Ill are cancelled out when the fluorescence ratios are calculated. I was able to detect translocation of $\beta$-lactamase-fused TARP by Yersinia enterocolitica and the SPI-2 system of Salmonella typhimurium. Co-expression with its cognate chaperone significantly increased TARP translocation by both species.

However, I was unable to detect any TARP translocation by enterropathogenic E.coli or by the SPI-1 system of S. typhimurium, even in the presence of Slc1. Secretion or translocation of chlamydial type III effectors had been previously reported with the SPI-1, SPI-2, and $Y$. eneterocolitica type III systems, but not with EPEC. One possible reason for the lack of TARP translocation by EPEC could be that its ATPase recognizes a different $\mathrm{N}$-terminal secretion signal. Alternatively, it could be that a chlamydial chaperone-mediated three-dimensional secretion signal cannot be recognized by the EPEC type III apparatus.

It has been previously shown that some chlamydial effectors are preferentially translocated by the heterologous SPI-1 or SPI-2 type III secretion systems[43]. Similarly, in our translocation assays I see a preference for translocation of TARP by the SPI-2 system. However, the fluorescence ratio had previously only been applied to measure the translocation of effectors within a few hours of infection, and the SPI-2 secretion system translocates effectors from its intracellular Salmonella containing vesicle between 6 and 10 hours post-infection[161]. I had to consider the possibility the some of the CCF2/AM substrate was able to access the non-translocated $\beta$-lactamase-fused effectors inside the 
intracellular Salmonella, thus leading to a background level of cleaved substrate in this assay. SPI-2 translocation of $\beta$-lactamase fused effectors had been demonstrated previously but instead of calculating a fluorescence ratio, they observed blue and green fluorescence by live-cell microscopy[159]. Their microscopy results revealed a low level of conversion of the green to blue signal, but they were still able to obviously distinguish between cells in which a $\beta$-lactamase fused effector had been translocated (blue) from cells with no translocation (green). This background level of cleavage falsely skews the fluorescence ratio to indicate translocation in the (Empty) $\beta$-lactamase vector only negative control. However, I still believe that the elevated fluorescence ratio for TARP in the presence of $\mathrm{Slcl}$ is significantly higher than this background, indicating actual chaperone-mediated translocation by the SPI-2 secretion system of S. typhimurium.

Secretion of TARP into media had been previously demonstrated by $Y$. pseudotuberculosis[42], and I was able to confirm Yersinia's ability to secrete TARP by Y. enterocolitica. However, TARP's translocation into eukaryotic cells had not previously been tested, and even though TARP is secreted into media without a chaperone, secretion and translocation of effectors by Yersinia has been shown to have differential requirements for chaperones[62]. It has been shown that for Yersinia effectors, the $\mathrm{N}$-terminal secretion signal is sufficient for secretion into low-calcium media, while translocation of effectors into host cells requires their cognate chaperones. This dogma is in agreement with our findings that TARP is secreted into media similarly to the positive control Yersinia effector YopH, but TARP requires Slc1 to be efficiently translocated into host cells. 
I performed phylogenetic analysis of cytoplasmic type III flagellar and nonflagellar ATPases from C.trachomatis and C.pneumoniae in comparison with the SPI-1 and SPI-2 ATPases from S.typhimurium, EscN from EPEC, and YscN from $Y$. enterocolitica to assess whether the relatedness of ATPases could explain TARP translocation differences. The chlamydial non-flagellar ATPase CdsN was more closely related to $\mathrm{YscN}$ than $\mathrm{InvC}$, SsaN, or EscN. This finding agrees with the fact that Y.enterocolitica was able to secrete and translocate TARP. The Slc1-dependence of TARP for efficient translocation of Yersinia indicates that the Slc1: TARP complex interacts with YscN in much the same way as it interacts with $\mathrm{CdsN}$ during chlamydial infection. Since TARP is capable of being translocated by the Salmonella SPI-2 type III secretion system but not by the SPI-1 system, it was interesting to note that the SPI-2 ATPase ssaN was more closely related to $\mathrm{CdsN}$ than the SPI-1 ATPase InvC. These phylogenetic comparisons of type III ATPases imply that the Slc1: TARP complex is also likely to be able to interact with chlamydial CdsN. The agreement between ATPase phylogeny and functional interaction with the effector TARP gives credibility to our heterologous translocation assays.

Taken together, these findings indicate a role for Slc1 in the translocation of TARP by the chlamydial type III secretion apparatus (Figure 38). 


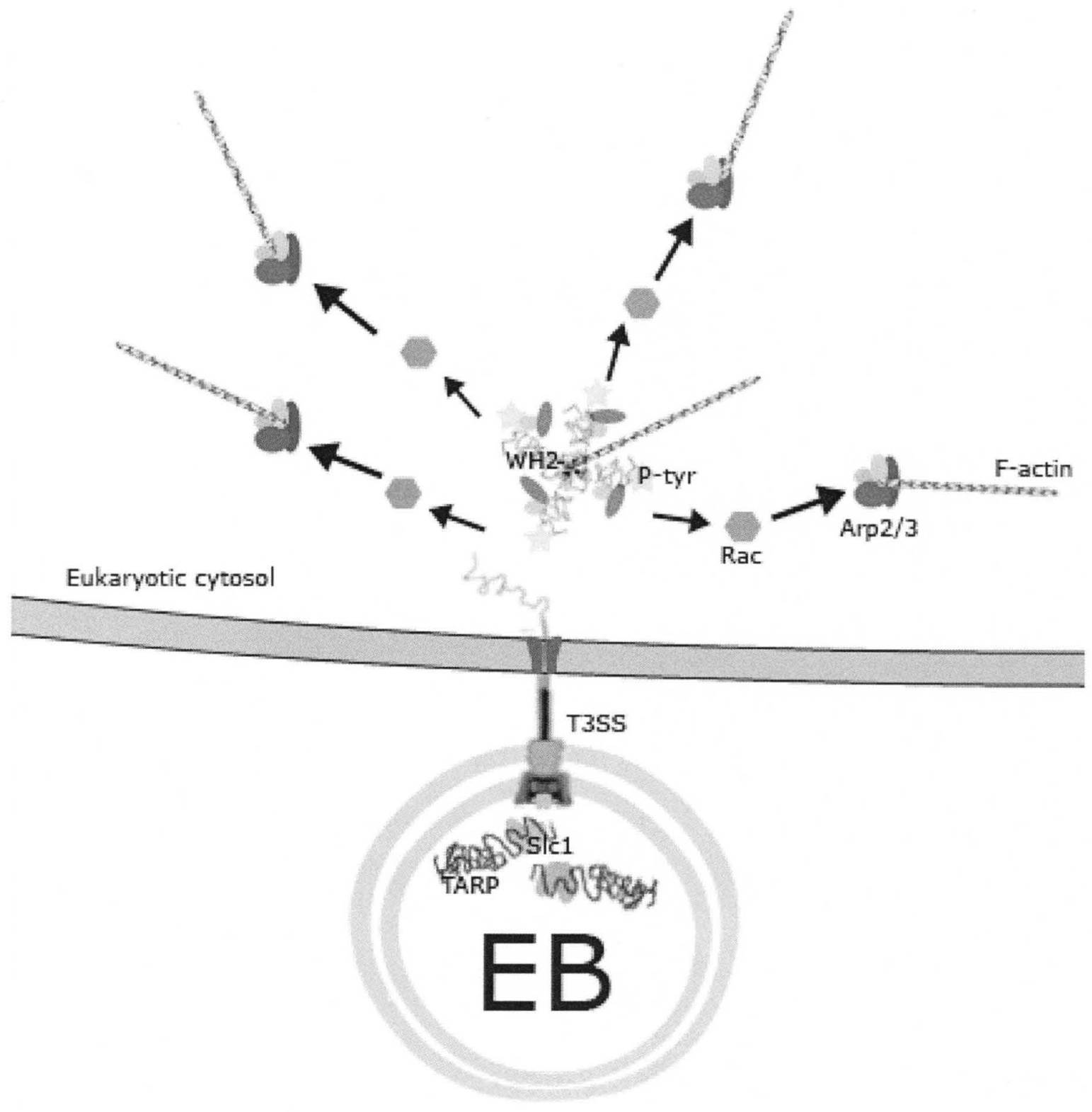

Figure 38. Model of Slc1-mediated translocation of TARP into eukaryotic cells. In the chlamydial EB, Slc1 associates with the N-terminus of TARP to reorganize its chaperone binding domain, which in turn is recognized by the type III secretion apparatus. Further unfolding by the ATPase allows TARP translocation through the type III secretion apparatus and into the eukaryotic cytosol where molecules of TARP form aggregates. Multiple domains on TARP are involved in actin polymerization that facilitates invasion of eukaryotic cells. TARP aggregates are nucleate actin at their WH2-like domains, while the tyrosine rich repeats become phosphorylated by host kinases. These phosphorylate repeats act as magnets for host signaling molecules and recruit further kinases guanine exchange factors that are involved in Rac activation and subsequent Arp2/3 formation. 


\section{CONCLUSIONS}

This dissertation presents the first indication of a type III secretion chaperone mediating the translocation of a chlamydial effector into the cytosol of its target host cell. Importantly, this translocated effector, TARP, has been shown to be essential for ensuring the efficient invasion of non-phagocytic cells and subsequently enabling Chlamydia to propagate. Not only does the requirement of the chaperone Slc1 in TARP translocation indicate a similar mechanism of effector translocation as detected in other type III-containing organisms, it also reveals the function of a previously uncharacterized protein, which is not an easy feat considering the genetically-intractable nature of Chlamydia spp.

Biochemical characterization of the specific interaction between SIc1 and TARP indicate a chaperone-mediated translocation signal that is likely recognized by a type III ATPase. Detection of this translocation by the heterologous Salmonella SPI-2 and $Y$. enterocolitica type III secretion systems but not by enterropathogenic E.coli or the Salmonella SPI-1 systems reveals a common mechanism for the chaperone-effector recognition by type III components. Comparison of the ATPases between these species indicates a role for the chlamydial ATPase $\mathrm{CdsN}$ in recognition of the Slc1: TARP complex. Further characterization the interaction of $\mathrm{CdsN}$ with this complex as well as other type III chaperone: substrate complexes may define a mechanism of differential 
selection of type III substrates for translocation such as the recognition of threedimensional chaperone-substrate translocation signals.

Recent work has demonstrated the inhibition of chlamydial growth and division by chemical inhibitors of type III secretion. The exact mechanism of this inhibition is unknown, and a likely target of these and future inhibition studies is the type III ATPase. Mapping the specific interactions that direct substrate recognition in the presence and absence of the type III chaperones will be pivotal to guiding the design of type III inhibitors for basic research and possibly for antibiotic use against several gram-negative pathogens.

The conservation of the type III secretion system among distantly related pathogenic and environmental Chlamydiaceae, all of which exhibit the biphasic intracellular lifestyle, indicates a role for the type III secretion system in maintaining this common lifestyle. Type III inhibitor studies will also provide valuable insight in the mechanisms directing the regulation of the Chlamydia's unique biphasic developmental cycle. Substrate-switching, chaperone-mediated translocation hierarchies, interaction of type III components with transcription machinery, and sequestering of type III chaperones or substrates are all potential mechanisms that Chlamydia spp. may exploit to ensure efficient survival and transitioning between infectious (EB) and replicating (RB) forms. 


\section{REFERENCES}

1. Darville, T. and T.J. Hiltke, Pathogenesis of genital tract disease due to Chlamydia trachomatis. J Infect Dis, 2010. 201 Suppl 2: p. S114-25.

2. Prevention, C.f.D.C.a., Chlamydia Prevalence Monitoring Project annual report 2007: sexually transmitted disease surveillance 2007 supplement. Atlanta, GA: Center for Disease Control and Prevention, 2009.

3. Organization, W.H., Global prevalence and incidence of selected curable sexually transmitted infections. Geneva, Switzerland: World Health Organization, 2001.

4. Millman, K., et al., Population-based genetic epidemiologic analysis of Chlamydia trachomatis serotypes and lack of association between ompA polymorphisms and clinical phenotypes. Microbes Infect, 2006. 8(3): p. 604-11.

5. Stothard, D.R., G. Boguslawski, and R.B. Jones, Phylogenetic analysis of the Chlamydia trachomatis major outer membrane protein and examination of potential pathogenic determinants. Infect Immun, 1998. 66(8): p. 3618-25.

6. Mabey, A.W.S.a.D.C., Trachoma. Clinical Evidence, 2003(10): p. 777-88.

7. Kuper, H., et al., A critical review of the $S A F E$ strategy for the prevention of blinding trachoma. Lancet Infect Dis, 2003. 3(6): p. 372-81.

8. Gaynor, B.D., et al., Community treatment with azithromycin for trachoma is not associated with antibiotic resistance in Streptococcus pneumoniae at 1 year. $\mathrm{Br} \mathrm{J}$ Ophthalmol, 2003.87(2): p. 147-8.

9. Grayston, J.T., Background and current knowledge of Chlamydia pneumoniae and atherosclerosis. J Infect Dis, 2000. 181: p. S402-S410.

10. Alp, C.W.a.N.J., Role of Chlamyida pneumoniae in atherosclerosis. Clinical Science, 2008. 114: p. 509-531.

11. Gottlieb, S.L., et al., Summary: The natural history and immunobiology of Chlamydia trachomatis genital infection and implications for Chlamydia control. J Infect Dis, 2010. 201 Suppl 2: p. S190-204.

12. Stephens, R.S., The cellular paradigm of chlamydial pathogenesis. Trends Microbiol, 2003. 11(1): p. 44-51.

13. Rasmussen, S.J., et al., Secretion of proinflammatory cytokines by epithelial cells in response to Chlamydia infection suggests a central role for epithelial cells in chlamydial pathogenesis. J Clin Invest, 1997. 99(1): p. 77-87.

14. Wyrick, P.B., Chlamydia trachomatis persistence in vitro: an overview. J Infect Dis, 2010. 201 Suppl 2: p. S88-95.

15. Batteiger, B.E., et al., Repeated Chlamydia trachomatis genital infections in adolescent women. J Infect Dis, 2010. 201(1): p. 42-51. 
16. Batteiger, B.E., et al., Protective immunity to Chlamydia trachomatis genital infection: evidence from human studies. J Infect Dis, 2010. 201 Suppl 2: p. S17889.

17. Gomes, J.P., et al., Correlating Chlamydia trachomatis infectious load with urogenital ecological success and disease pathogenesis. Microbes and Infection, 2006. 8(1): p. 16-26.

18. Kinnunen, A.H., et al., HLA DQ alleles and interleukin-10 polymorphism associated with Chlamydia trachomatis-related tubal factor infertility: a casecontrol study. Hum Reprod, 2002. 17(8): p. 2073-8.

19. Cohen, C.R., et al., Human immunodeficiency virus type 1-infected women exhibit reduced interferon-gamma secretion after Chlamydia trachomatis stimulation of peripheral blood lymphocytes. J Infect Dis, 2000. 182(6): p. 1672-7.

20. Debattista, J., et al., Reduced levels of gamma-interferon secretion in response to chlamydial $60 \mathrm{kDa}$ heat shock protein amongst women with pelvic inflammatory disease and a history of repeated Chlamydia trachomatis infections. Immunol Lett, 2002. 81(3): p. 205-10.

21. Ficarra, M., et al., A Distinct Cellular Profile Is Seen in The Human Endocervix During Chlamydia trachomatis Infection. American Journal of Reproductive Immunology, 2008. 60(5): p. 415-425.

22. Horn, M., et al., Illuminating the evolutionary history of chlamydiae. Science, 2004. 304(5671): p. 728-30.

23. McCoy AJ, M.A., Building the invisible wall: updating the chlamydial peptidoglycan anomaly. Trends Microbiol, 2006. 14: p. 70-77.

24. Cornelis, G.R. and F. Van Gijsegem, Assembly and function of type III secretory systems. Annual Review of Microbiology, 2000. 54: p. 735-774.

25. Arnold, R., et al., Sequence-based prediction of type III secreted proteins. PLoS Pathog, 2009. 5(4): p. el000376.

26. Beeckman, D.S.A. and D.C.G. Vanrompay, Bacterial Secretion Systems with an Emphasis on the Chlamydial Type III Secretion System. Current Issues in Molecular Biology, 2010. 12: p. 17-41.

27. Betts, H.J., et al., Bioinformatic and biochemical evidence for the identification of the type III secretion system needle protein of Chlamydia trachomatis. J Bacteriol, 2008. 190(5): p. 1680-90.

28. Kim, J.F., Revisiting the chlamydial type III protein secretion system: clues to the origin of type III protein secretion. Trends in Genetics, 2001. 17(2): p. 65-69.

29. Peters, J., et al., Type III secretion $\neq$ la Chlamydia. Trends in Microbiology, 2007. 15(6): p. 241-251.

30. Samudrala, R., F. Heffron, and J.E. McDermott, Accurate prediction of secreted substrates and identification of a conserved putative secretion signal for type III secretion systems. PLoS Pathog, 2009. 5(4): p. e1000375.

31. Yip, C.K. and N.C. Strynadka, New structural insights into the bacterial type III secretion system. Trends Biochem Sci, 2006. 31(4): p. 223-30.

32. Stone, C.B., Gulir, D.C., Gilchrist, J.D., Toor, R.K, and J.B. Mahony, Interactions between flagellar and type III secretion proteins in Chlamydia pneumoniae. BMC Microbiology, 2010. 
33. Lonrenzini, E., Singer, A., Singh, B., Lam, T., Skarina, T., Chirgadae, N.Y., Savchenko, A., Gupta, R.S., Structure and protein-protein interaction studeis on Chlamydia trachomatis protein CT670. J Bacteriol, 2010. 192(2746-2756).

34. Markham, A.P., Jaafar, Z.A., Kemege, K.E., Middaugh, C.R., \& Hefty, P.S., Biophysical characterization of Chlamydia trachomatis CT584 supports its potential role as a type III secretion needle tip protein. Biochemistry, 2009. 48(10353-10361).

35. Stone, C.B., et al., Characterization of the putative type III secretion ATPase $C d s N$ (Cpn0707) of Chlamydophila pneumoniae. Journal of Bacteriology, 2008. 190(20): p. 6580-6588.

36. Johnson, D.L., C.B. Stone, and J.B. Mahony, Interactions between $C d s D, C d s Q$, and CdsL, three putative Chlamydophila pneumoniae type III secretion proteins. Journal of Bacteriology, 2008. 190(8): p. 2972-2980.

37. Slepenkin, A., L.M. de la Maza, and E.M. Peterson, Interaction between components of the type III secretion system of Chlamydiaceae. J Bacteriol, 2005. 187(2): p. 473-9.

38. Fields, K.A., et al., Analysis of putative Chlamydia trachomatis chaperones Scc2 and Scc3 and their use in the identification of type III secretion substrates. $\mathrm{J}$ Bacteriol, 2005. 187(18): p. 6466-78.

39. Fields, K.A., D.J. Mead, C.A. Dooley \& T. Hackstadt, Chlamydia trachomatis type III secretion: evidence for a functional apparatus during early cycle development. Mol Microbiol, 2003. 48: p. 671-683.

40. Subtil, A., C. Parsot, and A. Dautry-Varsat, Secretion of predicted Inc proteins of Chlamydia pneumoniae by a heterologous type III machinery. Mol Microbiol, 2001. 39(3): p. 792-800.

41. Fields, K.A. and T. Hackstadt, Evidence for the secretion of Chlamydia trachomatis CopN by a type III secretion mechanism. Mol Microbiol, 2000. 38(5): p. 1048-60.

42. Clifton, D.R., et al., A chlamydial type III translocated protein is tyrosinephosphorylated at the site of entry and associated with recruitment of actin. Proc Natl Acad Sci U S A, 2004. 101(27): p. 10166-71.

43. Ho, T.D. and M.N. Starnbach, The Salmonella enterica serovar typhimuriumencoded type III secretion systems can translocate Chlamydia trachomatis proteins into the cytosol of host cells. Infect Immun, 2005. 73(2): p. 905-11.

44. Hueck, C.J., Type III secretion systems in bacterial pathogens of animals and plants. Microbiol Med Biol Rev, 1998. 62: p. 379-433.

45. Silvia-Herzog, E., Ferracci F., Jackson, M.W., Joseph, S.S., \& Plano, G.V., Membrane localization and topology of the Yersinia pestis YscJ lipoprotein. Microbiology, 2008. 154: p. 593-607.

46. Fields, H.J.B.-H.a.K.A., The chlamydial type III secretion mechanism: revealing cracks in a tough nut. Frontiers in Microbiology, 2010. 1(114): p. 1-13.

47. Johnson, D.L.J.B.M., Chlamydophila pneumoniae PknD exhibits dual amino acid specificity and phosphorylates Cpn0712, a putative type III secretion YscD homolog. J Bacteriol, 2007. 189(7549-7555).

48. Riordan, K.E.a.O.S., YscU cleavage and the assembly of Yersinia type III secretion machine complexes. Mol Microbiol, 208. 68: p. 1485-1501. 
49. Spaeth, K.E., Y.-S. Chen, and R.H. Valdivia, The Chlamydia Type III Secretion System C-ring Engages a Chaperone-Effector Protein Complex. PLoS Pathog, 2009. 5(9): p. e1000579.

50. Riordan, K.E., Sorg, I., Berube, B.J., and O. Scheewind, Impassable YscP substrates and their impact on the Yersinia enterocolitica type III secretion pathway. J Bacteriol, 2008.

51. Huang, J., Lesser, C.F., \& Lory, S., The essential role of the CopN protein in Chlamydia pneumoniae intracellular growth. Nature, 2008. 456: p. 112-115.

52. Pallen, M.J., S.A. Beatson, and C.M. Bailey, Bioinformatics, genomics and evolution of non-flagellar type-III secretion systems: a Darwinian perspective. FEMS Microbiol Rev, 2005. 29(2): p. 201-29.

53. Rao, X.P.D., Z. Hua, J. Wang, M. Luo, J. Wang, Y, Lang, G. Zhong, A. Hochschild \& L. Shen, A regulator from Chlamydia trachomatis modulates the activity of RNA polymerase through direct interaction with the beta subunit and the primary sigma subunit. Genes Dev, 2009. 23: p. 1818-1829.

54. Subtil, A., et al., A directed screen for chlamydial proteins secreted by a type III mechanism identifies a translocated protein and numerous other new candidates. Molecular Microbiology, 2005. 56(6): p. 1636-1647.

55. Galan, J.E. and H. Wolf-Watz, Protein delivery into eukaryotic cells by type III secretion machines. Nature, 2006. 444(7119): p. 567-73.

56. Ghosh, P., Process of protein transport by the type III secretion system. Microbiol Mol Biol Rev, 2004. 68(4): p. 771-95.

57. Feldman, M.F. and G.R. Cornelis, The multitalented type III chaperones: all you can do with $15 \mathrm{kDa}$. FEMS Microbiol Lett, 2003. 219(2): p. 151-8.

58. Page, A.L. and C. Parsot, Chaperones of the type III secretion pathway: jacks of all trades. Mol Microbiol, 2002. 46(1): p. 1-11.

59. Akeda, Y. and J.E. Galan, Chaperone release and unfolding of substrates in type III secretion. Nature, 2005. 437(7060): p. 911-5.

60. Gauthier, A. and B.B. Finlay, Translocated intimin receptor and its chaperone interact with ATPase of the type III secretion apparatus of enteropathogenic Escherichia coli. J Bacteriol, 2003. 185(23): p. 6747-55.

61. Parsot, C., C. Hamiaux, and A.L. Page, The various and varying roles of specific chaperones in type III secretion systems. Curr Opin Microbiol, 2003. 6(1): p. 714.

62. Boyd, A.P., I. Lambermont, and G.R. Cornelis, Competition between the Yops of Yersinia enterocolitica for delivery into eukaryotic cells: Role of the SycE chaperone binding domain of YopE. Journal of Bacteriology, 2000. 182(17): p. $4811-4821$.

63. Bryan Coburn, I.C., and B. Brett Finlay, Type III Secretion Systems and Disease. Clinical Microbiology Reviews, 2007: p. 535-549.

64. Bertelli, C., et al., The Waddlia genome: a window into chlamydial biology. PLoS One, 2010. 5(5): p. e10890.

65. Subtil, A., A directed screen for chlamydial proteins secreted by a type III mechanism identifies a translocated protein and numerous other new candidates. Mol Microbiol, 2005. 56: p. 1636-1647. 
66. Mehlitz, A.B., Sebastian; Hess, Simone; Selbach, Matthias; Meyer, Thomas F., Complex kinase requirements for Chlamydia trachomatis TARP phosphorylation. FEMS Microbiol Lett, 2008. 289: p. 233-240.

67. Lane, J.B.M., Charla; Al Khodor, Souhalia; Grieshaber, Scott S.; Carabeo, Rey A., Chlamydial Entry Involves TARP Binding of Guanine Nucleotide Exchange Factors. PLOS Pathogens, 2008. 4(3).

68. ElIll, C.A., Ceesay, Alhaji; Kim, Jung Hwa; Kalman, Daniel; Engel, Joanne, RNA Interference Screen Identifies Abl Kinase and PDGFR Signaling in Chlamydia trachomatis Entry. PLOS Pathogens, 2008. 4(3).

69. Carabeo, R.A.D., Cheryl A.; Grieshaber, Scott S. and Ted Hackstadt, Rac interacts with Abi-1 and WAVE2 to promote an Arp2/3-dependent actin recruitment during chlamydial invasion. Cellular Microbiology, 2007.9(9): p. 2278-2288.

70. Jewett, T.J., et al., Chlamydial TARP is a bacterial nucleator of actin. Proc Natl Acad Sci U S A, 2006. 103(42): p. 15599-604.

71. Clifton D.R., D.C., Grieshaber SS, Carabeo RA, Fields KA, Hackstadt T, Phosphorylation of the chlamydial effector protein TARP is species specific and not required for the recruitment of actin. Infection and Immunity, 2005. 73(7): p. 3860-3868.

72. Carabeo, R.A., et al., Requirement for the Rac GTPase in Chlamydia trachomatis invasion of non-phagocytic cells. Traffic, 2004. 5(6): p. 418-25.

73. Carabeo, R.A., et al., Chlamydia trachomatis induces remodeling of the actin cytoskeleton during attachment and entry into HeLa cells. Infect Immun, 2002. 70(7): p. 3793-803.

74. Belland, Y.M.A.R.J., The chlamydial developmental cycle. FEMS Microbiology Reviews, 2005. 29: p. 949-959.

75. Valdivia, R.H., Chlamydia effector proteins and new insight into chlamydial cellular microbiology. Current Opinion in Microbiology, 2008. 11: p. 53-59.

76. Scidmore-Carlson, M.A., Shaw, E.I., Dooley, C.A., Fischer, E.R., and Hackstadt, $\mathrm{T}$., Identification and characterization of a Chlamydia trachomatis early operon encoding four novel inclusion membrane proteins. Mol Microbiol, 1999. 33: $\mathrm{p}$. 753-765.

77. Rzomp, K.A., Moorhead, A.R., and Scidmore, M.A., The GTPase Rab4 interacts with Chlamydia trachomatis inclusion membrane protein CT229. Infect Immun, 2006. 74: p. 5362-5373.

78. Cortes, C., Rzomp KA, Tvinnereim A, Scidmore MA, Wizel B, Chlamydia pneumoniae inclusion membrane protein Cpn0585 interacts with multiple Rab GTPases. . Infect Immun, 2007. 75: p. 5586-5596.

79. Grieshaber, S.S., Grieshaber, N. A., and Hackstadt, T., Chlamydia trachomatis uses host cell dynein to traffic to the microtubule-organizing center in a p50 dynamitin-independent process. J Cell Science, 2003. 116: p. 3793-3802.

80. Scidmore, M.A., and Hackstadt, T., Mammalian 14-3-3beta associates with Chlamydia trachomatis inclusion membrane via its interaction with IncG. Mol Microbiol, 2001. 39: p. 1638-1650. 
81. Verbeke, P., Welter-Stahl, L., Ying, S., Hansen, J., Hacker, G., Darville T., Ojcuis, D.M. , Recruitment of BAD to the Chlamydia trachomatis vacuole correlates with host-cell survival. PLOS Pathogens, 2006. 2(45).

82. Balsara, Z.R., Misaghi, S., Lafave, J.N., Starnbach, M.N., Chlamydia trachomatis infection induces cleavage of the mitotic cyclin B1. Infect Immun, 2006. 74: p. 5602-5608.

83. Pirbhai, M., Dong, F., Zhong, Y., Pan, K.Z., Zhong, G., The secreted protease factor CPAF is responsible for degrading pro-apoptotic BH3-only proteins in Chlamydia trachomatis-infected cells. J Biol Chem, 2006. 281: p. 31495-31501.

84. Betts, H.J., K. Wolf, and K.A. Fields, Effector protein modulation of host cells: examples in the Chlamydia spp. arsenal. Current Opinion in Microbiology, 2009. 12(1): p. 81-87.

85. Karyagina, A.S., et al., Effector proteins of chlamydiae. Molecular Biology, 2009. 43(6): p. 897-916.

86. Wolf, K., G.V. Plano, and K.A. Fields, A protein secreted by the respiratory pathogen Chlamydia pneumoniae impairs IL-17 signaling via interaction with human Act1. Cell Microbiol, 2009.

87. Wilson, D.P., et al., Kinematics of intracellular chlamydiae provide evidence for contact-dependent development. J Bacteriol, 2009. 191(18): p. 5734-42.

88. Hoare, A., et al., Spatial constraints within the chlamydial host cell inclusion predict interrupted development and persistence. BMC Microbiol, 2008. 8: p. 5.

89. Abdel Rahman YM, B.R., The chlamydial developmental cycle. FEMS Microbiol Rev, 2005. 29: p. 949-959.

90. Case, E.D., E.M. Peterson, and M. Tan, Promoters for Chlamydia type III secretion genes show a differential response to DNA supercoiling that correlates with temporal expression pattern. J Bacteriol, 2010. 192(10): p. 2569-74.

91. Ouellette, S.P., et al., The Chlamydia pneumoniae type III secretion-related lcr H gene clusters are developmentally expressed operons. J Bacteriol, 2005. 187(22): p. 7853-6.

92. Nicholson, T.L., et al., Global stage-specific gene regulation during the developmental cycle of Chlamydia trachomatis. J Bacteriol, 2003. 185(10): p. 3179-89.

93. Belland, R.J., et al., Genomic transcriptional profiling of the developmental cycle of Chlamydia trachomatis. Proc Natl Acad Sci U S A, 2003. 100(14): p. 8478-83.

94. Chen, J.C., J.P. Zhang, and R.S. Stephens, Structural requirements of heparin binding to Chlamydia trachomatis. J Biol Chem, 1996. 271(19): p. 11134-40.

95. Gutierrez-Martin, C.B., et al., Heparin-mediated inhibition of Chlamydia psittaci adherence to HeLa cells. Microb Pathog, 1997. 22(1): p. 47-57.

96. Su, H. and H.D. Caldwell, Sulfated polysaccharides and a synthetic sulfated polymer are potent inhibitors of Chlamydia trachomatis infectivity in vitro but lack protective efficacy in an in vivo murine model of chlamydial genital tract infection. Infect Immun, 1998. 66(3): p. 1258-60.

97. Rasmussen-Lathrop, S.J., et al., Chlamydia-dependent biosynthesis of a heparan sulphate-like compound in eukaryotic cells. Cell Microbiol, 2000. 2(2): p. 137-44. 
98. Stephens, R.S., et al., Eukaryotic cell uptake of heparin-coated microspheres: a model of host cell invasion by Chlamydia trachomatis. Infect Immun, 2000. 68(3): p. 1080-5.

99. Yabushita, H., et al., Effects of chemically modified heparin on Chlamydia trachomatis serovar L2 infection of eukaryotic cells in culture. Glycobiology, 2002. 12(5): p. 345-51.

100. Stephens, R.S., J.M. Poteralski, and L. Olinger, Interaction of Chlamydia trachomatis with mammalian cells is independent of host cell surface heparan sulfate glycosaminoglycans. Infect Immun, 2006. 74(3): p. 1795-9.

101. Yan, Y., et al., Inhibitory effect of heparan sulfate-like glycosaminoglycans on the infectivity of Chlamydia pneumoniae in HL cells varies between strains. Microbes Infect, 2006. 8(3): p. 866-72.

102. Su, H., et al., Differential effect of trypsin on infectivity of Chlamydia trachomatis: loss of infectivity requires cleavage of major outer membrane protein variable domains II and IV. Infect Immun, 1988. 56(8): p. 2094-100.

103. Su, H., et al., Chlamydia trachomatis-host cell interactions: role of the chlamydial major outer membrane protein as an adhesin. Infect Immun, 1990. 58(4): p. 1017-25.

104. Su, H. and H.D. Caldwell, In vitro neutralization of Chlamydia trachomatis by monovalent Fab antibody specific to the major outer membrane protein. Infect Immun, 1991. 59(8): p. 2843-5.

105. Swanson, A.F. and C.C. Kuo, Binding of the glycan of the major outer membrane protein of Chlamydia trachomatis to HeLa cells. Infect Immun, 1994. 62(1): p. 24-8.

106. Kuo, C., et al., An N-linked high-mannose type oligosaccharide, expressed at the major outer membrane protein of Chlamydia trachomatis, mediates attachment and infectivity of the microorganism to HeLa cells. J Clin Invest, 1996. 98(12): p. 2813-8.

107. Chen, J.C. and R.S. Stephens, Chlamydia trachomatis glycosaminoglycandependent and independent attachment to eukaryotic cells. Microb Pathog, 1997. 22(1): p. 23-30.

108. Swanson, A.F. and C.C. Kuo, The 32-kDa glycoprotein of Chlamydia trachomatis is an acidic protein that may be involved in the attachment process. FEMS Microbiol Lett, 1994. 123(1-2): p. 113-7.

109. Carabeo, R.A. and T. Hackstadt, Isolation and characterization of a mutant Chinese hamster ovary cell line that is resistant to Chlamydia trachomatis infection at a novel step in the attachment process. Infect Immun, 2001. 69(9): p. 5899-904.

110. Puolakkainen, M., C.C. Kuo, and L.A. Campbell, Chlamydia pneumoniae uses the mannose 6-phosphate/insulin-like growth factor 2 receptor for infection of endothelial cells. Infect Immun, 2005. 73(8): p. 4620-5.

111. ElIll, C.C., A; Kim, JH; Kalman, D; Engel, JN, RNA interference screen identifies Abl kinase and PDGFR signaling in Chlamydia trachomatis entry. PLOS PATHOGENS, 2008. 4(3).

112. Wang, A., et al., A systemic network for Chlamydia pneumoniae entry into human cells. J Bacteriol, 2010. 192(11): p. 2809-15. 
113. Ajonuma, L.C., et al., CFTR is required for cellular entry and internalization of Chlamydia trachomatis. Cell Biol Int, 2010. 34(6): p. 593-600.

114. Fadel, S. and A. Eley, Differential glycosaminoglycan binding of Chlamydia trachomatis OmcB protein from serovars $E$ and $L G V$. J Med Microbiol, 2008. 57(Pt 9): p. 1058-61.

115. Molleken, K., E. Schmidt, and J.H. Hegemann, Members of the Pmp protein family of Chlamydia pneumoniae mediate adhesion to human cells via short repetitive peptide motifs. Mol Microbiol, 2010. 78(4): p. 1004-17.

116. Conant, C.G. and R.S. Stephens, Chlamydia attachment to mammalian cells requires protein disulfide isomerase. Cell Microbiol, 2007. 9(1): p. 222-32.

117. Abromaitis, S. and R.S. Stephens, Attachment and entry of Chlamydia have distinct requirements for host protein disulfide isomerase. PLoS Pathog, 2009. 5(4): p. e1000357.

118. Hackstadt, T., Identification and properties of chlamydial polypeptides that bind eucaryotic cell surface components. J Bacteriol, 1986. 165(1): p. 13-20.

119. Inman, W.M. and R.U. Meuser, Chlamydia trachomatis elementary bodies possess proteins which bind to eucaryotic cell membranes. J Bacteriol, 1986. 165(2): p. 602-7.

120. Su, H., et al., A recombinant Chlamydia trachomatis major outer membrane protein binds to heparan sulfate receptors on epithelial cells. Proc Natl Acad Sci U S A, 1996. 93(20): p. 11143-8.

121. Zhang, J.P. and R.S. Stephens, Mechanism of C. trachomatis attachment to eukaryotic host cells. Cell, 1992. 69(5): p. 861-9.

122. Taraktchoglou, M., et al., Infectivity of Chlamydia trachomatis serovar LGV but not $E$ is dependent on host cell heparan sulfate. Infect Immun, 2001. 69(2): p. 968-76.

123. Fadel, S. and A. Eley, Chlorate: a reversible inhibitor of proteoglycan sulphation in Chlamydia trachomatis-infected cells. J Med Microbiol, 2004. 53(Pt 2): p. 935.

124. Stephens, R.S., et al., Heparin-binding outer membrane protein of chlamydiae. Mol Microbiol, 2001. 40(3): p. 691-9.

125. Moelleken, K. and J.H. Hegemann, The Chlamydia outer membrane protein $O m c B$ is required for adhesion and exhibits biovar-specific differences in glycosaminoglycan binding. Mol Microbiol, 2008. 67(2): p. 403-19.

126. Swanson, A.F., et al., Human mannose-binding protein inhibits infection of HeLa cells by Chlamydia trachomatis. Infect Immun, 1998. 66(4): p. 1607-12.

127. Chen, J.C. and R.S. Stephens, Trachoma and LGV biovars of Chlamydia trachomatis share the same glycosaminoglycan-dependent mechanism for infection of eukaryotic cells. Mol Microbiol, 1994. 11(3): p. 501-7.

128. Davis, C.H. and P.B. Wyrick, Differences in the association of Chlamydia trachomatis serovar $E$ and serovar $L 2$ with epithelial cells in vitro may reflect biological differences in vivo. Infect Immun, 1997. 65(7): p. 2914-24.

129. Birkelund, S., H. Johnsen, and G. Christiansen, Chlamydia trachomatis serovar L2 induces protein tyrosine phosphorylation during uptake by HeLa cells. Infect Immun, 1994. 62(11): p. 4900-8. 
130. Fawaz, F.S., et al., Infection with Chlamydia trachomatis alters the tyrosine phosphorylation and/or localization of several host cell proteins including cortactin. Infect Immun, 1997. 65(12): p. 5301-8.

131. Subtil, A., et al., Analysis of Chlamydia caviae entry sites and involvement of Cdc42 and Rac activity. J Cell Sci, 2004. 117(Pt 17): p. 3923-33.

132. Jewett, T.J.D., Cheryl; Mead, David J.; Ted Hackstadt, Chlamydia trachomatis TARP is phosphorylated by src family tyrosine kinases. Biochemical and Biophysical Research Communications, 2008. 371: p. 339-344.

133. Campellone, K.a.J.L., Nck-independent actin assembly is mediated by two phosphorylated tyrosines within enteropathogenic Escherichia coli Tir. Mol Microbiol, 2005. 56: p. 416-432.

134. Phillips, N.S., Hayward, R., and Koronakis V., Phosphorylation of the enterropathogenic E. coli receptor by the Src-family kinase c-Fyn triggers actin pedestal formation. Nat. Cell Biol., 2004. 6: p. 618-625.

135. Zhou, D.a.J.G., Salmonella entry into host cells: the work in concert of tyoe III secreted effector proteins. Microbes Infect., 2001. 3: p. 1293-1298.

136. Mehlitz, A., et al., TARP regulates early Chlamydia-induced host cell survival through interactions with the human adaptor protein SHC1. J Cell Biol, 2010. 190(1): p. 143-57.

137. Thalmann, J., et al., Actin re-organization induced by Chlamydia trachomatis serovar D--evidence for a critical role of the effector protein CT166 targeting Rac. PLoS One, 2010. 5(3): p. e9887.

138. HoIr, S., K. Wolf, and K.A. Fields, Evidence that CT694 is a novel Chlamydia trachomatis T3S substrate capable of functioning during invasion or early cycle development. Mol Microbiol, 2009. 72(6): p. 1423-37.

139. Virok, D.P.N., D.E., Whitmire, W.M., Crane, D.D.; Goheen, M.M.; and Caldwell, H.D., Chlamydial infection induces pathobiotype-specific protein tyrosine phosphorylation in epithelial cells. Infect Immun, 2005. 73: p. 1939-1946.

140. Swanson, K.C., DD; Caldwell, HD, Chlamydia trachomatis Species-Specific Induction of Ezrin Tyrosine-Phosphorylation Functions in Pathogen Entry. Infect Immun, 2007: p. 5669-5677.

141. Skoudy, A.J.M., A.; Aruffo, H; Ohayon, P.; Gounon, P.; Sansonetti, P. and Tran Van Nhieu., CD44 binds to the Shigella IpaB protein and participates in bacterial invasion of epithelial cells. Cell Microbiol, 2000. 2: p. 19-33.

142. contributors, O., Beta-Galactosidase Assay (A better Miller), in OpenWetWare. 2009 Feb 23.

143. Vandahl, B.B., S. Birkelund, and G. Christiansen, Genome and proteome analysis of Chlamydia. Proteomics, 2004. 4(10): p. 2831-42.

144. Skipp, P., et al., Shotgun proteomic analysis of Chlamydia trachomatis. Proteomics, 2005. 5(6): p. 1558-73.

145. Shaw, A.C., et al., Comparative proteome analysis of Chlamydia trachomatis serovar A, D and L2. Proteomics, 2002. 2(2): p. 164-86.

146. Olsen, A.W., et al., Identification of CT521 as a frequent target of Th1 cells in patients with urogenital Chlamydia trachomatis infection. Journal of Infectious Diseases, 2006. 194(9): p. 1258-1266. 
147. Creasey, E.A., et al., CesT is a bivalent enteropathogenic Escherichia coli chaperone required for translocation of both Tir and Map. Mol Microbiol, 2003. 47(1): p. 209-21.

148. Karimova, G., et al., A bacterial two-hybrid system that exploits a cAMP signaling cascade in Escherichia coli, in Methods in Enzymology. 2000, Academic Press. p. 59-73.

149. Karimova, G., J. Pidoux, A. Ullman, and D. Landant, Protein-protein interaction between Bacillus stearothermophilus Tyrosyl-tRNA Synthetase subdomains revealed by a Bacerial Two-Hybrid System. J. Mol Microbiol. Biotechnol. , 2001. 3(1): p. 73-82.

150. Fields, K.A., et al., Chlamydia trachomatis type III secretion: evidence for a functional apparatus during early-cycle development. Mol Microbiol, 2003. 48(3): p. 671-83.

151. Birtalan, S.C., R.M. Phillips, and P. Ghosh, Three-dimensional secretion signals in chaperone-effector complexes of bacterial pathogens. Mol Cell, 2002. 9(5): p. 971-80.

152. Abe, A., et al., Enteropathogenic Escherichia coli translocated intimin receptor, Tir, requires a specific chaperone for stable secretion. Molecular Microbiology, 1999. 33(6): p. 1162-1175.

153. Wilharm, G., et al., On the role of specific chaperones, the specific ATPase, and the proton motive force in type III secretion. Int J Med Microbiol, 2007. 297(1): p. 27-36.

154. Deng, W., et al., Regulation of type III secretion hierarchy of translocators and effectors in attaching and effacing bacterial pathogens. Infect Immun, 2005. 73(4): p. 2135-46.

155. Wulff-Strobel, C.R., A.W. Williams, and S.C. Straley, LcrQ and SycH function together at the Ysc type III secretion system in Yersinia pestis to impose a hierarchy of secretion. Mol Microbiol, 2002. 43(2): p. 411-23.

156. Karlinsey, J.E., et al., Translation/secretion coupling by type III secretion systems. Cell, 2000. 102(4): p. 487-97.

157. Stone, C.B., et al., Interactions between flagellar and type III secretion proteins in Chlamydia pneumoniae. Bmc Microbiology, 2010. 10: p. -.

158. Rodgers, L., et al., A Solvent-Exposed Patch in Chaperone-Bound YopE Is Required for Translocation by the Type III Secretion System. Journal of Bacteriology, 2010. 192(12): p. 3114-3122.

159. Thomas, N.A., et al., CesT is a multi-effector chaperone and recruitment factor required for the efficient type III secretion of both LEE- and non-LEE-encoded effectors of enteropathogenic Escherichia coli. Mol Microbiol, 2005. 57(6): p. 1762-79.

160. Diaz, M.H. and A.R. Hauser, Pseudomonas aeruginosa cytotoxin ExoU is injected into phagocytic cells during acute pneumonia. Infect Immun, 2010. 78(4): p. 1447-56.

161. Geddes, K., F. Cruz, and F. Heffron, Analysis of cells targeted by Salmonella type III secretion in vivo. PLoS Pathog, 2007. 3(12): p. e196.

162. Ashida, H., et al., Shigella chromosomal IpaH proteins are secreted via the type III secretion system and act as effectors. Mol Microbiol, 2007. 63(3): p. 680-93. 
163. Marches, O., et al., Characterization of two non-locus of enterocyte effacementencoded type III-translocated effectors, NleC and NleD, in attaching and effacing pathogens. Infect Immun, 2005. 73(12): p. 8411-7. 
CURRICULUM VITAE

Amanda J Brinkworth, Ph.D

\begin{tabular}{|c|c|c|c|}
\hline \multicolumn{4}{|l|}{ EDUCATION } \\
\hline INSTITUTION AND LOCATION & DEGREE & $\mathrm{MM} / \mathrm{YY}$ & FIELD OF STUDY \\
\hline Indiana University, Bloomington, IN & B.S. & $05 / 06$ & Biology \\
\hline $\begin{array}{l}\text { University of Louisville School of } \\
\text { Medicine, Louisville, KY }\end{array}$ & M.S & $12 / 07$ & Microbiol \& Immunology \\
\hline $\begin{array}{l}\text { University of Louisville School of } \\
\text { Medicine, Louisville, KY }\end{array}$ & Ph.D. & $01 / 11$ & Microbiology \\
\hline
\end{tabular}

POSITIONS AND EMPLOYMENT

2004-2006 Research Assistant, Department of Biology, Indiana University, Bloomington, IN

2006-pres Ph.D. Candidate, Dept of Microbiology and Immunology, University of Louisville, School of Medicine

2008-2010 Research Assistant, Centre for Molecular Microbiology and Infection, Imperial College London

\section{PROFESSIONAL MEMBERSHIPS}

2006-pres Member, Chlamydia Basic Research Society

2007-pres Member, European Society for Chlamydia Research

2010-pres Member, American Society for Microbiology

HONORS

2002-2003

2006-2008

2007

2007

$2007, ' 10$

IU STARS program, Indiana University IPIBS fellowship, University of Louisville, University of Louisville International Center Travel Grant, University of Louisville School of Medicine Research Committee Travel Grant, University of Louisville Graduate School Council Travel Grant, University of Louisville

\section{CONFERENCE POSTER PRESENTATIONS}

2008 Sixth meeting of the European Society for Chlamydia Research. University of Aarhus, Aarhus, Denmark, July 1-4

2009 Research! Louisville, University of Louisville, October 20-22

$2010 \quad 110^{\text {th }}$ General meeting of American Society for Microbiology, San Diego, May 23-27

SEMINAR PRESENTATIONS

2010 Department of Biological Sciences Seminar Series, University of Montana, Oct 11

\section{RESEARCH PUBLICATIONS}

Amanda J. Brinkworth, Antonio Tedim Pedrosa, Katarzyna Roguska, Sevanna Shahbazian, James E. Graham, Richard D. Hayward, Rey A. Carabeo. The type III chaperone Slcl mediates translocation of the C. trachomatis invasion-associated effector TARP. (Submitted to PLOS PATHOGENS, Feb 2011) 